\title{
Spectral Optical Monitoring of the Narrow Line Seyfert 1 galaxy Ark 564
}

\author{
A.I. Shapovalova ${ }^{1}$, L.Č. Popović ${ }^{2,3}$, A.N. Burenkov ${ }^{1}$, V.H. Chavushyan ${ }^{4}$, D. Ilić ${ }^{5,3}$, \\ A. Kovačević ${ }^{5,3}$, W. Kollatschny ${ }^{6}$, J. Kovačević, ${ }^{2,3}$, N.G. Bochkarev ${ }^{7}$, J. R. Valdes ${ }^{4}$, \\ J. Torrealba ${ }^{4}$, J. León-Tavares ${ }^{8}$, A. Mercado ${ }^{9}$, E. Benítez ${ }^{10}$, L. Carrasco ${ }^{4}$, D. Dultzin ${ }^{10}$ \\ and E. de la Fuente ${ }^{11}$ \\ ashap@sao.ru
}


Received _ ; accepted _

${ }^{1}$ Special Astrophysical Observatory of the Russian AS, Nizhnij Arkhyz, KarachaevoCherkesia 369167, Russia

${ }^{2}$ Astronomical Observatory, Volgina 7, 11160 Belgrade 74, Serbia

${ }^{3}$ Isaac Newton Institute of Chile, Yugoslavia Branch, Belgrade, Serbia

${ }^{4}$ Instituto Nacional de Astrofísica, Óptica y Electrónica, Apartado Postal 51-216, 72000 Puebla, Puebla, México

${ }^{5}$ Department of Astronomy, Faculty of Mathematics, University of Belgrade, Studentski trg 16, 11000 Belgrade, Serbia

${ }^{6}$ Institut für Astrophysik, Georg-August-Universität Göttingen, Germany

${ }^{7}$ Sternberg Astronomical Institute, Moscow, Russia

${ }^{8}$ Aalto University Metsähovi Radio Observatory, Metsähovintie 114, FIN-02540 Kylmälä, Finland

${ }^{9}$ Universidad Politécnica de Baja California, Av. de la Industria 291, 21010 Mexicali, B.C., México

${ }^{10}$ Instituto de Astronomía, Universidad Nacional Autónoma de México, Apartado Postal 70-264, México, D.F. 04510, México

${ }^{11}$ Instituto de Astronomía y Meteorología, Dpto. de Física CUCEI, Universidad de Guadalajara, Av. Vallarta 2602, 44130 Guadalajara, Jalisco, México 


\begin{abstract}
We present the results of a long-term (1999-2010) spectral optical monitoring campaign of the active galactic nucleus (AGN) Ark 564, which shows a strong Fe II line emission in the optical. This AGN is a narrow line Seyfert 1 (NLS1) galaxies, a group of AGNs with specific spectral characteristics. We analyze the light curves of the permitted $\mathrm{H} \alpha, \mathrm{H} \beta$, optical Fe II line fluxes, and the continuum flux in order to search for a time lag between them. Additionally, in order to estimate the contribution of iron lines from different multiplets, we fit the $\mathrm{H} \beta$ and Fe II lines with a sum of Gaussian components. We found that during the monitoring period the spectral variation $\left(\mathrm{F}_{\max } / \mathrm{F}_{\min }\right)$ of Ark 564 was between 1.5 for $\mathrm{H} \alpha$ to 1.8 for the Fe II lines. The correlation between the Fe II and $\mathrm{H} \beta$ flux variations is of higher significance than that of $\mathrm{H} \alpha$ and $\mathrm{H} \beta$ (whose correlation is almost absent). The permitted-line profiles are Lorentzian-like, and did not change shape during the monitoring period. We investigated, in detail, the optical Fe II emission and found different degrees of correlation between the Fe II emission arising from different spectral multiplets and the continuum flux. The relatively weak and different degrees of correlations between permitted lines and continuum fluxes indicate a rather complex source of ionization of the broad line emission region.
\end{abstract}

Subject headings: galaxies: active - galaxies: quasar - galaxies: individual (Ark 564) - line: profiles 


\section{Introduction}

Narrow-line Seyfert 1 (NLS1) galaxies were first introduced as a class of active galactic nuclei (AGNs) by Osterbrock \& Pogge (1985). Their optical spectra show relatively narrow $\left(\right.$ FWHM $\left.\leq 2000 \mathrm{~km} \mathrm{~s}^{-1}\right)$ permitted lines, which are narrower than in a typical Seyfert 1 galaxy. In particular, Osterbrock \& Pogge (1985) did show that, the permitted lines are only slightly broader than the forbidden ones, and that a strong Fe II emission is present in the optical region of the spectrum. In addition, the $[\mathrm{O}$ III] $\lambda 5007 / \mathrm{H} \beta$ ratio, emitted in the narrow line region (NLR), varies from 1 to 5 (Rodríguez-Ardila et al. 2000), instead of the universally adopted observed value for Seyfert 1s of around 10 (Rodríguez-Ardila et al. 2000), indicative of the presence of high-density gas. Osterbrock \& Pogge (1985) pointed out that the $\mathrm{H} \beta$ equivalent widths in NLS1s are smaller than typical values for normal Seyfert 1s, suggesting that they are not just normal Seyfert 1s seen at a particular viewing angle. Renewed interest in NLS1s arises from the discovery of their distinctive X-ray properties: they show a steep X-ray excess with a photon index of 3 below $100 \mathrm{keV}$, a steep hard X-ray continuum, and a rapid large-amplitude X-ray variability on timescales of minutes to hours (see Leighly 1999a,b, 2000; Panessa et al. 2011, and references therein). Moreover, optical studies have established that NLS1s lie at one end of the Boroson \& Green (1992) eigenvector 1 (EV1) and that they show a relatively strong Fe II emission and a weak [O III] emission (Boller et al. 1996). They also represent the "extreme Population A" objects (FWHM H $\beta<4000 \mathrm{~km} / \mathrm{s}$ ) as defined by the four dimensional eigenvector 1 (4DE1) in Sulentic et al. (2007); Marziani et al. (2010). 4DE1 involves four parameters and NLS1 are "extreme" in all of them: they have the narrowest broad $\mathrm{H} \beta$, strongest Fe II emission, strongest X-ray excess and largest C IV blueshifts.

Arakelian 564 (Ark 564, IRAS 22403+2927, MGC +05-53-012) is a bright $V=14.6$ mag. (de Vaucouleurs et al. 1991), nearby narrow-line Seyfert 1 galaxy $(z=0.02467)$, with 
an X-ray luminosity $L_{2-10 \mathrm{keV}}=2.4 \times 10^{43}, \mathrm{erg} \mathrm{s}^{-1}$ (Turner et al. 2001). This AGN is one of the brightest NLS1s in the X-ray band (Boller et al. 1996; Collier et al. 2001; Smith et al. 2008), and it shows a soft excess below $\sim 1.5 \mathrm{keV}$ and a peculiar emission-line-like feature at $0.712 \mathrm{keV}$ in the source rest frame (Chiho et al. 2004). The variations of the X-ray amplitude in the short-timescale light curve is very similar to those in the long-timescale light curve (Pounds et al. 2001), that is in contrast to the stronger amplitude variability on longer timescales, which is a characteristic of broad-line Seyfert 1 (BLS1) galaxies. In the UV part of the spectrum, this galaxy shows intrinsic UV absorption lines (Crenshaw et al. 1999). In order to explore the variability characteristics of a NLS1 in different wavelength bands, a multiwavelength monitoring campaign of Ark 564 was conducted (Shemmer et al. 2001). The optical campaign covered the periods 1998 November - 1999 November and 2000 May - 2001 January, where the object was observed both photometrically (UBVRI filters) and spectrophotometrically (spectral coverage 4800-7300 A) (Shemmer et al. 2001). The data set and analysis is described in details and compared with the simultaneous X-ray and UV campaigns (Shemmer et al. 2001). The results of this intensive variability multiwavelength campaign show that the optical continuum is not significantly correlated with the X-ray emission (Shemmer et al. 2001). The UV campaign, carried out with the HST on 2000 May 9 and 2000 July 8, is described in Collier et al. (2001). These authors found a small fractional variability amplitude of the continuum between $1365 \AA$ and $3000 \AA$ (around $6 \%$ ), but reported that large-amplitude short-timescale flaring behavior is present, with trough-to-peak flux changes of about $18 \%$ in approximately 3 days (Collier et al. 2001). The wavelength-dependent continuum time delays in Ark 564 have been detected and these delays may indicate a stratified continuum reprocessing region (Collier et al. 2001).

Here we present the long-term monitoring of Ark 564 in the optical part of the spectrum. We analyzed the variability in the permitted emission lines and continuum in 
order to determin the size and structure of emitting regions of the permitted Balmer and Fe II lines. We placed particular interest in the strong Fe II lines of the $\mathrm{H} \beta$ spectral region whose behavior is investigated in details and discussed in this paper.

The paper is organized as follow: in section 2 we describe the observations and data reduction procedures, in section 3 we give an analysis of the spectral data, in section 4 we explore the correlations between different lines and the continuum, as well as between different lines, in section 5 we investigate in more details the Fe II variation, in section 6 we discuss our results, and in section 7 we provide our conclusions.

\section{Observations and data reduction}

Spectral monitoring of Ark 564 was carried out with the $6 \mathrm{~m}$ and $1 \mathrm{~m}$ telescopes of the SAO RAS (Russia, 1999-2010), the INAOE's $2.1 \mathrm{~m}$ telescope of the "Guillermo Haro Observatory" (GHO) at Cananea, Sonora, México (1999-2007), and the $2.1 \mathrm{~m}$ telescope of the Observatorio Astronómico Nacional at San Pedro Martir (OAN-SPM), Baja California, México (2005-2007). Spectra were taken with long-slit spectrographs equipped with CCDs. The typical observed wavelength range was 4000-7500 , the spectral resolution was $\mathrm{R}=5-15 \AA$, and with $\mathrm{S} / \mathrm{N}$ ratio $>50$ in the continuum near $\mathrm{H} \alpha$ and $\mathrm{H} \beta$. In total 100 blue and 55 red spectra were obtained during 120 nights. In the analysis, about $\mathbf{1 0 \%}$ of spectra were discarded for several different reasons: e.g. a) large noise (S/N<15A - 2001 Aug 29 (blue, red), 2001 Oct 08 (blue), 2001 Oct 09 (blue, red), taken with Zeiss $(1 \mathrm{~m})+\operatorname{CCD}(1 \mathrm{k} \times 1 \mathrm{k})$; b) large noise and badly corrected spectral sensitivity in the blue part - 2006 Jun 28 (blue, red), 2006 Aug 29(blue), 2006 Aug 30 (blue), 2009 Aug 14 (blue), 2009 Oct 11 (blue), taken with Zeiss $(1 \mathrm{~m})+\operatorname{CCD}(2 \mathrm{k} \times 2 \mathrm{k})$. We note here that the $\operatorname{CCD}(2 \mathrm{k} \times 2 \mathrm{k})$ sensitivity in the blue part is not good enough, since it is a red CCD; c) poor spectral 
resolution ( $\mathrm{R}>20 \AA$, - 2003 Nov 18, 2004 Oct 18, taken with $2.1 \mathrm{~m}$ GHO). Thus our final data set consisted of 91 blue and 50 red spectra, which were used in further analysis.

From 1999 to 2003 spectral observations with $1 \mathrm{~m}$ Zeiss telescope of the SAO were carried out with two different CCDs (formats used were $1 \mathrm{k} \times 1 \mathrm{k}$ or $530 \times 580$ ) and the $\mathrm{H} \alpha$ and $\mathrm{H} \beta$ spectral regions were observed separately. From 2004 to 2010 a CCD $(2 \mathrm{k} \times 2 \mathrm{k}$, EEV CCD42-40) was used, allowing us to observe the entire wavelength range (4000-8000) $\AA$ with a spectral resolution of 8-10 $\AA$. However, this CCD in the blue part of some spectra presents large sensitivity variations (i.e. bad S/N), which are badly corrected, thus the blue region of these spectra was not used in our analysis.

From 2004 to 2007, the spectral observations with two Mexican $2.1 \mathrm{~m}$ telescopes were carried out with two observational setups. In the case of GHO observations we used the following configuration: 1) with a grating of $150 \mathrm{l} / \mathrm{mm}$ (spectral resolution of $\mathrm{R}=15 \AA$, a resolution similar to the observations of 1999-2003); 2) with a grating of 300 $\mathrm{l} / \mathrm{mm}$ (moderate spectral resolution of $\mathrm{R}=7.5 \AA$ ). The similar spectral characteristics at the OAN-SPM were, respectively, obtained with the following configuration: 1) with a grating of $300 \mathrm{l} / \mathrm{mm}$ (spectral resolution of $\mathrm{R}=15 \AA$ ); 2) with a grating of $600 \mathrm{l} / \mathrm{mm}$ (moderate spectral resolution of $\mathrm{R}=7.5 \AA)$.

As a rule, observations were carried out with the moderate resolution in the blue or red bands during the first night of each run. In order to cover $\mathbf{H} \alpha$ and $\mathbf{H} \beta$ at the same time, we used the lower resolution mode and observed the entire spectral range 4000-7500 $\AA$; and then the moderate resolution was adopted again for the following night. Since the shape of the continuum of active galaxies practically does not change during adjacent nights, it was easy to match, the blue and red bands obtained with the moderate resolution in different nights. To this aim we used the data obtained for the continuum from the 
low-dispersion spectra for the entire wavelength range. By this procedure, the photometric accuracy is thus considerably improved with respect to a match obtained by overlapping the extremes of the blue and red continuum (3-5\% instead of 5-10\%).

Spectrophotometric standard stars were observed every night.

Information on the source of spectroscopic observations is listed in Table 1. The log of the spectroscopic observations is given in Table 2. Taking into account all observations, the mean sampling rate is 33.20 , and the median rate is 2.95 days. The big difference between the mean and median sampling rate is due to the big gaps in the variability campaign.

The spectrophotometric data reduction was carried out either with the software developed at the SAO RAS or with the IRAF package for the spectra obtained in México. The image reduction process included bias and flat-field corrections, cosmic ray removal, 2D wavelength linearization, sky spectrum subtraction, addition of the spectra for every night, and relative flux calibration based on observations of standard star.

\subsection{Absolute calibration (scaling) of the spectra}

The standard technique of flux calibration of the spectra (i.e. comparison with stars of known spectral energy distribution) is not precise enough for the study of AGN variability, since even under good photometric conditions, the accuracy of spectrophotometry is usually not better than $10 \%$. Therefore we used standard stars only to provide a relative flux calibration. Instead, for the absolute calibration, the observed fluxes of the forbidden, narrow emission lines are adopted for the scaling procedure the AGN spectra, since these

fluxes are expected to be constant (Peterson 1993). From HST observations (Crenshaw et al. 2002) it was shown that the NLR in Ark 564 is about $0.2 "$ (95 pc), and this facts implies 
a constant [OIII] $\lambda 5007$ flux intensity during several hundred years. Consequently, the flux of this forbidden line should not have changed during our monitoring period. The scaling of the blue spectra was performed using the method of Van Groningen \& Wanders (1992) modified by Shapovalova et al. (2004)1. We will not repeat the scaling procedure here, we only note that the flux in the lines was determined after subtraction of a linear continuum determined by the beginning and the end of a given spectral interval. This method allowed us to obtain a homogeneous set of spectra with the same wavelength calibration and the same $[\mathrm{OIII}] \lambda 5007$ flux. The $[\mathrm{OIII}] \lambda 5007$ flux in absolute units was taken from Shemmer et al. (2001): $\mathrm{F}([\mathrm{OIII}] \lambda 5007)=(2.4 \pm 0.1) \times 10^{-13} \mathrm{erg} \mathrm{s}^{-1} \mathrm{~cm}^{-2}$. The spectra, obtained with $2.1 \mathrm{~m}$ telescopes in Mexico with a resolution of $12-15 \AA$, containing both $\mathrm{H} \alpha$ and $\mathrm{H} \beta$ regions were scaled using the $[\mathrm{O}$ III $] \lambda 5007$ line. However, some spectra of Ark 564 were obtained separately in the blue $(\mathrm{H} \beta)$ and red $(\mathrm{H} \alpha)$ wavelength bands, with a resolution of 8-10 A. Usually, the red edge of the blue spectra and the blue edge of the red spectra overlap in an interval of $300 \AA$. Therefore, first the red spectra (17) were scaled using the overlapping continuum region with the blue ones. The latter were scaled with the $[\mathrm{OIII}] \lambda 5007$ line. In these cases the scaling uncertainty was about 5\%-10\%. Then scaling of the red spectra was refined using the mean flux in $[\mathrm{OI}] \lambda 6300$ (mean $\left.\mathrm{F}[\mathrm{OI}] \lambda 6300 \sim(1.93 \pm 0.24) \times 10^{-14}\right)$, determined from low-dispersion spectra $(\mathrm{R} \sim 12-15$ $\AA$ ). For 3 red spectra (JD:2452886.9; 2455058.5 and 245116.4) we have no blue spectrum in adjacent nights, and they was scaled using only the mean flux of the $[\mathrm{OIII}] \lambda 6300 \AA$ line.

\footnotetext{
${ }^{1}$ see Appendix A Shapovalova et al. (2004)
} 


\subsection{Unification of the spectral data}

In order to investigate the long term spectral variability of an AGN, it is necessary to conform a consistent, uniformed data set. Since observations were carried out with 4 different instruments, we must correct the line and continuum fluxes for aperture effects (Peterson \& Collins 1983). To this effect, we determined a point-source correction factor $\varphi$ given by the following expression (see Peterson et al. 1995, for a detailed discussion):

$$
F(\mathrm{H} \beta)_{\text {true }}=\varphi \cdot F(\mathrm{H} \beta)_{\mathrm{obs}}
$$

where $F(\mathrm{H} \beta)_{\text {obs }}$ is the observed $\mathrm{H} \beta$ flux; $F(\mathrm{H} \beta)_{\text {true }}$ is the aperture corrected $\mathrm{H} \beta$ flux. The contribution of the host galaxy to the continuum flux depends also on the aperture size. The continuum fluxes $F(5235 \AA$ ) (in the observed-frame) were corrected for different amounts of host-galaxy contamination, according to the following expression (see Peterson et al. $(1995))$ :

$$
F(5235 \AA)_{\text {true }}=\varphi \cdot F(5235 \AA)_{\text {obs }}-G(g),
$$

where $F(5235 \AA)_{\text {obs }}$ is the continuum flux at $\mathbf{5 2 3 5} \AA$ in the observed-frame; $G(g)$ is an aperture-dependent correction factor to account for the host galaxy contribution. The GHO observing scheme (Table 1), which correspond to a projected aperture $\left(2.5^{\prime \prime} \times 6^{\prime \prime}\right)$ of the $2.1 \mathrm{~m}$ telescope, were adopted as standard (i.e. $\varphi=1.0, G(g)=0$ by definition). The correction factors $\varphi$ and $G(g)$ are determined empirically by simulated aperture photometry of suitable images of the narrow line emission and the starlight of the host galaxy in the same way as it is given in Peterson et al. (1995). This procedure is accomplished empirically by comparing pairs of simultaneous observations from each of given telescope data sets to that of the standard data set (as it used in AGN Watch, e.g. Peterson et al. 1994, 1999, 2002). As noted in these papers, even after scaling of the spectra to a common value of the [OIII] 5007 flux, there are systematic differences between 
the light curves produced from the data obtained with different telescopes. Therefore, it is proposed to correct for small offsets between the light curves from different sources in a simple, but effective fashion (e.g. Peterson et al. 2002, and references therein), attributing these small relative offsets to aperture effects (Peterson et al. 1995). The procedure also corrects for other unidentified systematic differences between data sets (for example, miscentering of the AGN nucleus in spectrograph aperture, etc.). In our paper we took the GHO-data as standard, because this data set contains the largest number of observed spectra. The correction factors $\varphi$ and $G(g)$ are determined empirically by comparing pairs of nearly simultaneous observations from each of the given telescope data sets $(\mathrm{L}(\mathrm{U}), \mathrm{SPM}, \mathrm{Z1K}, \mathrm{Z} 2 \mathrm{~K})$ to that of the GHO data set. In practice, intervals which we defined as "nearly simultaneous" are typically of 1-2 days. Therefore, the variability on short time scales $(<2$ days) is suppressed. The point-source correction factors $\varphi$ and $G(g)$ values for different samples are listed in Table 3, Using these factors, we re-calibrated the observed fluxes of $\mathrm{H} \alpha, \mathrm{H} \beta$, Fe II 48,49 and continuum to a common scale corresponding to our standard aperture $2.5^{\prime \prime} \times 6^{\prime \prime}$ (Table 4).

\subsection{Measurements of the spectra and errors}

From the scaled spectra we determined the average flux in the blue continuum at the rest-frame wavelength $\sim 5100 \AA$, by means of flux averages in the spectral interval 5094-5123 $\AA$ in the rest-frame (Table 5). We also calculated the average flux in the red continuum at the rest-frame wavelength $\sim 6200 \AA$, by averaging the flux in the spectral interval $6178-6216 \AA$ in the rest-frame (Table 5). These intervals wavelength were selected because they do not contain noticeable emission lines (Fe II or any other lines, see Fig. 1). 
In order to determine the observed fluxes of the $\mathrm{H} \alpha, \mathrm{H} \beta$ and Fe II lines we need to subtract the underlying continuum, thus, a linear continuum was defined through $20 \AA$ windows, located at rest-frame wavelengths $4762 \AA$ ( $4880 \AA$ in the observed-frame) and $5123 \AA$ ( $5250 \AA$ in the observed-frame) for the $\mathrm{H} \beta$ line, and at rest-frame wavelengths $6334 \AA(6490 \AA$ in the observed-frame) and $6656 \AA(6820 \AA$ in the observed-frame) for the $\mathrm{H} \alpha$ line (Fig. 11). In the case of Fe II emission, a precise subtraction of the underlying continuum for a larger wavelength range is required. Hence, a polynomial fit for the continuum was drawn through continuum windows (Fig. 2) located at the rest-frame wavelength intervals $4210-4230 \AA, 5080-5100 \AA, 5600-5630 \AA$, (see e.g. Kuraszkiewicz et al. 2002; Kovačević et al. 2010).

After the continuum subtraction, we measured the observed fluxes of the emission lines in the following rest-frame wavelength intervals: $4817-4909 \AA$ for $\mathrm{H} \beta, 6480-6646 \AA$ for H $\alpha$, and 5100-5470 $\AA$ for the Fe II emission (hereafter Fe II red shelf). The measurements are given in Table 5. In this Fe II wavelength range, mainly the 48 and 49 Fe II multiplets are located (Fig. 2), yet there is also a contribution of the 42 multiplet around $5170 \AA$ in the rest-frame (see Kovačević et al. 2010). This spectral interval was chosen because the Fe II lines there included are not blended with other either strong broad and narrow emission lines (e.g. He II $4686 \AA$ ). This allows to determine the Fe II line fluxes in a straightforward manner (Fig. 2) . Further in the text, we discuss a more detailed analysis of the Fe II emission in a wider spectral interval 4100-5600 $\AA$ in the rest-frame (see Section $5)$.

Worth noting, is the fact that the $\mathrm{H} \beta$ and $\mathrm{H} \alpha$ fluxes here reported, include the corresponding narrow component fluxes: in the case of $\mathrm{H} \beta$ - only the narrow $\mathrm{H} \beta$ is included (the $[\mathrm{OIII}] \lambda \lambda 4959,5007$ lines are out from the $\mathrm{H} \beta$ spectral interval); while for $\mathrm{H} \alpha$ case - lines of $[\mathrm{NII}] \lambda \lambda 6548,6584$ and narrow $\mathrm{H} \alpha$ are included. As fluxes of narrow lines are 
assumed to be constant, they have no influence on the broad line component variability. The line and continuum fluxes were corrected for aperture-effect using the listed correction factors in Table 3 (see Subsection 2.2).

In Table 4 the fluxes for the blue continuum (at $5100 \AA$ ), $\mathrm{H} \alpha, \mathrm{H} \beta$ and Fe II lines are listed. We have also estimated the flux contribution from the $\mathrm{H} \beta$ and $\mathrm{H} \alpha$ narrow components and $[\mathrm{NII}] \lambda \lambda 6548$, 6584, from multi Gaussian fit to the blends $(\mathrm{H} \beta+[\mathrm{OIII}] \lambda \lambda 4959,5007$ and $\mathrm{H} \alpha+[\mathrm{NII}] \lambda \lambda 6548,6584)$ of the mean profiles. The best fits are plotted in Fig. 3. From the mean spectra, the estimated contribution of $\mathrm{F}(\mathrm{H} \beta)$ narrow component to the total line flux is $\sim 20 \%$. The narrow $\mathrm{F}(\mathrm{H} \alpha)$ has a contribution of about $30 \%$ while the fluxes of the $[\mathrm{NII}] \lambda \lambda 6548,6584$ a $7 \%$ one. A similar result (an averaged contribution of $\sim 18 \%$ ) was obtained for the $\mathrm{F}(\mathrm{H} \beta)$ narrow component from the gaussian fit to every blue spectrum (see section 5).

Additionally, we measured line-segment fluxes. In doing this, we divided the $\mathrm{H} \alpha$ and $\mathrm{H} \beta$ line profiles into three parts: a blue wing, a core and a red wing. The adopted intervals in wavelength and velocity are listed in Table 5 .

The mean uncertainties (errorbars) for the fluxes of continuum, $\mathrm{H} \alpha$ and $\mathrm{H} \beta$ lines, and their line segments (wings and core) are listed in Table 5. These quantities were estimated from the comparison of the results of spectra obtained within a time interval shorter than 3 days. The details of evaluation techniques of these uncertainties (errorbars) are given in Shapovalova et al. (2008). As can be notices, in Table 5 the mean error of the continuum flux, total $\mathrm{H} \alpha, \mathrm{H} \beta$ lines and their cores, is $\sim 4 \%$. While due to their relatively weaker flux, the errors in the determination of the fluxes of the Fe II and line wings are larger, about $\sim(7-9) \%$. 


\section{Results of the data analysis}

\subsection{Variability of the emission lines and of the optical continuum}

We analyzed flux variations in the continuum and emission lines from a total of 91 spectra covering the $\mathrm{H} \beta$ wavelength region, and 50 spectra covering the $\mathrm{H} \alpha$ line vicinity. In Fig. 4 the blue continuum subtracted spectrum of Ark 564, obtained with the $6 \mathrm{~m}$ SAO telescope in November 23, 2001 (JD 2452237.1) is presented. There, we marked the positions of some relevant Fe II multiplets $(27,28,37,38,42,48,49)$ and other important emission lines. As it can be easily noticed, the Fe II emission is rather strong, as it is usually the case in NLS1 galaxies.

From the flux data listed in Table 4, we obtained light curves for the blue and red continua, and for $\mathrm{H} \alpha, \mathrm{H} \beta$, and Fe II emission (Fig. [5), and their line-segments (blue wing, core, and red wing, Fig. 6). As one can see in Figs. 5 and 6 , the fluxes declined slowly from the beginning to the end of the monitoring period. For $\mathrm{H} \alpha$ and $\mathrm{H} \beta$ a decline of $\sim 20 \%$ is present, while, for the Fe II emission, a decline of $\sim 30 \%$ and one of $\sim 40 \%$ for the continuum flux are seen (Fig. 5). In the upper panel of Fig. 5, the upper dashed line represents the flux at the beginning of the monitoring campaign, and the lower dashed line at the end of it. There is only one red point in 2010 at which point the red flux increased (two lower panels in Fig. 51). Similarly as in Collier et al. (2001), the light curves show several flare-like increments (see Fig. 5). The light curves of line wings and core (Fig. 6) show practically simultaneous variations. There might be up to five flare-like events detected in our data (see Table 6) when the flux increases $\sim 10-20 \%$ for a short period of time $(\sim 1-3$

days, Table 6), out of which two flares were prominent: in December 2003 and August 2004. As it can be seen from Table 6, as a rule, flare-like events in the continuum (see dF(cnt) in \%) are stronger than in emission lines. 
Long-term flux-monitoring programs have shown that the flux variations of AGNs tend to be stochastic (i.e., there are few cases of periodicity or quasiperiodicity, see e.g. Shapovalova et al. 2010). However, the AGN light curves sometimes, as in the case of Ark 564, can show a flare-like characteristics whose spectral properties are consistent with a shot-noise process (Cruise \& Dodds 1985; Hufnagel \& Bregman 1992; Hughes et al. 1992). One way to reproduce shot noise is through a superposition of a series of identical impulses, occurring at intervals dictated by Poisson statistics. In a Poisson process, the overall rate of events is statistically constant, yet the starting times of individual events are independent of all previous ones. The time intervals between events follow an exponential distribution. It is possible to use such a process in Ark 564 variability investigation, by assuming a constant flare rate $\rho$, and let $T j$ be the occurrence time of the $j$-th flare. Probability of no occurrence of flare in the interval $[T j, T j+\tau]$ is $\exp (-\rho \tau)$.

The probability that a second flare will occur within a time $\tau$ after the first one is $p(\tau)=1-\exp (-\rho \tau)$. Actually, we can say that $p[T n+1-T n<$ $\tau \mid T 0, T 1, \ldots T n]=1-\exp (-\rho \tau)$ means that at least one flare does occur between $T n$ and $T n+\tau$.

As it can be seen from Table 6 , in the continuum and $\mathrm{H} \beta$ we have 4 events in 4 separate years which gives a density of events in 10-year long monitoring period of 0.4 events per year. In such a way we could estimate probability of time between flare events (which could be recorded in the continuum flux and $\mathbf{H} \beta$ line) as $p(\tau)=1-\exp (-0.4 \tau)$. As for $\mathbf{H} \alpha$, we have $\mathbf{3}$ events in 3 separate years over 10 years period, which leads to $p(\tau)=1-\exp (-0.3 \tau)$. Finally, in the case of Fe II line we have 5 events in 4 separate years ( 2 events occurred 
at the end of October 2006), so we could take $\rho=3 / 10+2 / 10=1 / 2$, which leads to $p(\tau)=1-\exp (-0.5 \tau)$. The exponential density is monoton decreasing; hence there is a high probability of a short interval, and a small probability of a long interval between flares. This means that typically we will have flares occurring close to each other and spaced out by long, but rare intervals with no occurrence of flares.

In Table 7 we list several parameters characterizing the variability of the continuum, total line, and line-segments fluxes. There are several methods to estimate variability, here we will use the method given by O'Brien et al. (1998). There $F$ denotes the mean flux over the whole observing period and $\sigma(F)$ its standard deviation. $R(\max / \mathrm{min})$ is the ratio of the maximal to minimal fluxes in the monitoring period. $F(\operatorname{var})$ is a inferred (uncertainty-corrected) estimate of the variation amplitude with respect to the mean flux, defined as:

$$
F(\operatorname{var})=\left[\sqrt{\sigma(F)^{2}-e^{2}}\right] / F(\text { mean })
$$

$e^{2}$ being the mean square value of the individual measurement uncertainty for $\mathrm{N}$ observations, i.e. $e^{2}=\frac{1}{N} \sum_{i}^{N} e(i)^{2}$ (O'Brien et al. 1998).

From Table 7 one can see that the amplitude of variability $F($ var) is $\sim 10 \%$ for the continuum and Fe II emission and $\sim 7.5 \%$ for the total $\mathrm{H} \beta$ flux. The $\mathrm{H} \beta$ blue wing shows slightly larger variability $(\mathrm{F}(\mathrm{var}) \sim 15 \%$ ) than the red one $(\mathrm{F}(\mathrm{var}) \sim 11 \%$, see Table 7). However, the $\mathrm{H} \alpha$ line wings and core show lower amplitude variability ( $\mathrm{F}(\mathrm{var}) \sim 8 \%)$ than the $\mathrm{H} \beta$ wings $(\mathrm{F}(\operatorname{var}) \sim(11-15) \%)$. 


\subsection{Mean and Root-Mean-Square Spectra}

We calculated the mean $\mathrm{H} \alpha$ and $\mathrm{H} \beta$ line profiles and their root-mean-square (rms) profiles. To find the different portion of variability in different line parts, as much as it is possible, first we inspect the spectra and conclude that the spectra with spectral resolution $\leq 11 \AA$ for $\mathrm{H} \alpha$ and $\leq 10 \AA$ for $\mathrm{H} \beta$ are good enough for this purpose, thus having a sample of 23 red spectra and 61 blue spectra (Fig. 7). For this purposes the spectra were calibrated to have the same spectral resolution ( $11 \AA$ for $\mathrm{H} \alpha$ and $10 \AA$ for $\mathrm{H} \beta$ ).

Fig. 7 shows that the $\mathrm{H} \alpha$ and $\mathrm{H} \beta$ line profiles in Ark 564 are Lorentzian like (with

broad wings), that is a characteristic of the NLS1 galaxies (see e.g. Sulentic et al. 2009, 2011). The rms profile of $\mathrm{H} \beta$ resembles a Lorentzian like one, while in case of $\mathrm{H} \alpha$ there is practically no change in the profile. The FWHM of the $\mathrm{H} \beta$ line from the observed mean and rms profiles is $960 \mathrm{~km} / \mathrm{s}$, and from the observed mean profile of $\mathrm{H} \alpha$ is $800 \mathrm{~km} / \mathrm{s}$. The full width at Zero Intensity (FWOI) of $\mathrm{H} \beta$ is much more difficult to measure since the Fe II emission contributes to the red wing. Thus we only give estimates of FWOI of $\mathrm{H} \beta$ mean profiles (or rms) to be $\sim 8000$ and for $\mathrm{H} \alpha$ is also $\sim 8000 \mathrm{~km} / \mathrm{s}$. As it can be seen in Fig. 7 , the rms is relatively weak $\left(F_{\mathrm{rms}}(\mathrm{H} \alpha) / F_{\mathrm{H} \alpha} \sim 0.01\right.$ and $\left.F_{\mathrm{rms}}(\mathrm{H} \beta) / F_{\mathrm{H} \beta} \sim 0.07\right)$, meaning that there are no significant changes in the line profiles of $\mathrm{H} \alpha$ and $\mathrm{H} \beta$ during the monitoring period. Note here that we did a recalibration of the $\mathbf{H} \beta$ line taking that the [OIII] lines have the same profile during the monitoring period, therefore we have small $\mathrm{rms}$ in forbidden lines, but it is also interesting that the rms shape of $\mathrm{H} \beta$ is practically the same as the total $\mathrm{H} \beta$ (composed from the broad and narrow components, see Fig. 3). This may indicate that the whole (Lorentzian-like) line is emitted from a complex BLR and that the contribution of the narrow component, that is coming from the same region as the [OIII] lines, is negligible. 


\section{On the other hand, the $\mathrm{H} \alpha$ line rms shows that the variability is caused mainly by variations in the line wings.}

\section{The continuum vs. line flux correlations}

To determine whether there are any changes in the structure of the broad-line region (BLR), we investigated both the relationships between the total line flux of $\mathrm{H} \alpha, \mathrm{H} \beta$ and Fe II and different line-segments (wings and core) of $\mathrm{H} \alpha$ and $\mathrm{H} \beta$.

In Fig. 8 the correlations between the total line flux of $\mathrm{H} \beta, \mathrm{H} \alpha$ and Fe II are presented. It is interesting to note that the correlations between the flux variation of $\mathrm{H} \beta$ and $\mathrm{H} \alpha$ is significantly weaker $(\mathrm{r} \sim 0.40$, and it seems statistically insignificant with $\mathrm{P}=0.0053)$ than that with the Fe II $\left(\mathrm{r} \sim 0.58\right.$, and $\left.\mathrm{P}<10^{-8}\right)$. The lack of correlations between the $\mathrm{H} \alpha$ and $\mathrm{H} \beta$ fluxes may indicate a very complex BLR structure. On the other hand, the correlation between different line-segment fluxes (i.e. blue/red wing-core, blue wing-red wing) are better, especially for $\mathrm{H} \alpha$ (Fig. 9).

In Fig. 10 we present the relationships between the continuum flux at $6200 \AA$ (for $\mathrm{H} \alpha$ )

and $5100 \AA$ (for $\mathrm{H} \beta$ ) and the total line and line-segment fluxes for $\mathrm{H} \alpha$ and $\mathrm{H} \beta$. As it can be seen in Fig. 10 the correlation between line and continuum fluxes are weak. Such weak linear correlations of the lines with continuum may indicate existence of different sources of ionization (AGN source - photo-ionization, shock-impact excitation and etc.). It is interesting to note that the Fe II emission seems to show a slightly better correlation with the continuum at $5100 \AA\left(\mathrm{r} \sim 0.76\right.$, and $\left.\mathrm{P}<10^{-16}\right)$ than Balmer lines (Fig. 11). 


\subsection{Balmer decrement}

We have calculated the $\mathrm{BD}=\mathrm{F}(\mathrm{H} \alpha) / \mathrm{F}(\mathrm{H} \beta)$ flux ratio, i.e. the Balmer decrement (see Fig. 12), using 50 blue and red spectra taken in the same night (or one night before or after). We obtained a mean Balmer decrement value $\mathrm{BD}($ mean $)=4.396 \pm 0.369$, and no significant changes in the monitoring period. In Fig. 12 the BD against the continuum flux is shown, and it can be seen there is no correlation between the BD and continuum ( $\mathrm{R} \sim 0.02)$. It is apparent that the BD was more or less constant during the 11-year monitoring period. The ratio of $\mathrm{H} \alpha$ and $\mathrm{H} \beta$ depends on the physics in the BLR, and in the low density regime, the $\mathrm{H} \alpha / \mathrm{H} \beta$ ratio is expected to be below 4 and it has a slight dependence on temperature (see discussion and Figs. 6 and 7 in

Ilić et al. 2012). In the high density regime, the $\mathrm{H} \alpha / \mathrm{H} \beta$ ratio starts to depend on the temperatrue (see Ilić et al. 2012). The obtained mean BD for Ark 564 seems to be close to the high density regime, and changes in the BD from 3.5 to 5.5 might be caused by an inhomogeneous BLR, i.e. may indicate a stratified BLR in density, temperature and rate of ionization.

\subsection{Lags between continuum and permitted lines}

In order to determine potential time lags between the continuum and permitted line changes, we calculated the cross-correlation function $(\mathrm{CCF})$ for the continuum light curve with the emission-line light curves. There are several ways to construct a CCF, and it is always advisable the use of two or more methods to confirm the obtained results. Therefore, we cross-correlated the $5100 \AA$ continuum light curve with both the $\mathrm{H} \beta$ and $\mathrm{H} \alpha$ line (and Fe II emission) light curves using two methods: (i) the z-transformed discrete correlation function (ZDCF) method introduced by Alexander (1997), and (ii) the interpolation cross-correlation function method (ICCF) described by Bischoff \& Kollatschny (1999). 
The time lags calculated by ZDCF are given in Table 8 , where it can be seen that inferred lags have large associated errors. It is interesting to note that the Fe II lines tend to have shorter lag values, while the longest one is that of the $\mathrm{H} \alpha$ line. If one takes a direct conversion from the time lag to the BLR size, the expected BLR sizes are of an order of $10^{-3}$ parsecs ( 0.003 to 0.0055 pc) that indicate a compact BLR, but also a strong stratification in the emitting region of Ark 564, where the Fe II emitting region tends to be very compact and the largest one is the H $\alpha$ emitting line region. We also calculated the lags using the ICCF and found a delay between the continuum and $\mathrm{H} \beta$ line of $\sim 6.7$ days, and between the continuum and Fe II emission of about 0 days, that is in agreement with the ZDCF method (see Table 8). The errors are around 10 lightdays.

The uncertainties in the delays inferred from the CCFs are difficult to estimate, especially the evaluation of a realistic error of the CCF. In our case, the main problem in the time delay determination, likely comes from the small variation detected in lines and continuum fluxes (see Table 7) and also their weak correlations (see Figs. 10 and 11). Therefore, all obtained lag times should be taken with caution.

\section{Variation of the Fe II lines}

As mentioned above, the strong Fe II emission is one of the main characteristics for NLS1 galaxies. Optical Fe II $(\lambda \lambda 4400-5400)$ emission is one of the most interesting features in AGN spectra. The emission arises from numerous transitions of the complex Fe II ion (see Kovačević et al. 2010, for more details). The iron emission is seen in almost all type-1 AGN spectra and it is especially strong in the NLS1s. The origin of the optical Fe II lines, their excitation mechanisms, and the spatial location of the Fe II emission region in AGNs are still open questions (see e.g. Popović et al. 2009; Kovačević et al. 2010; 
Popović \& Kovačević 2011). There are also many correlations between the Fe II emission and other AGN properties which require a physical explanation. As discussed above, we found that Fe II lines show a slightly better correlation with the continuum at $5100 \AA$ than Balmer lines (Fig. 11). On the other hand, it seems that the $\mathrm{H} \beta$ line flux is better correlated with the Fe II emission, than with $\mathrm{H} \alpha$. We should note here that this better correlation might be caused by the fact that the Fe II fluxes are much more susceptible to the contamination from the continuum emission than the $\mathrm{H} \alpha$ and H $\beta$ lines.

In order to explore the variability of Fe II lines in more detail, we fitted the Fe II emission complex by the multi-gaussian fitting method that Kovačević et al. (2010) and Popović \& Kovačević (2011) have described in detail. Our spectra cover a wider wavelength range $(\lambda \lambda 4100-5600 \AA)$. Hence, simultaneous fits of the Fe II template and $\mathrm{H} \delta, \mathrm{H} \gamma, \mathrm{He}$ II $\lambda 4686$, and $\mathrm{H} \beta$ line were carried out. Additionally to the Fe II line template introduced in Kovačević et al. (2010), we included here 17 other Fe II lines, basically the transition arising from two groups with lower levels ${ }^{4} \mathrm{P}$ and ${ }^{2} \mathrm{H}^{2}$ (see Fig 13 and Table 9]).

An example of a best fit is presented in Fig 14, where all the strong emission lines and Fe II features are labeled. Since the gaussian best-fit includes large number of free parameters (see Fig 14), we focused our attention in the fit required to reproduce as close as possible the Fe II line emission. To this aim, first we fit the strong hydrogen and helium lines, and correct their contribution to the Fe II lines, and then we apply the best-fit procedure to the Fe II emission. With this scheme, we substract the emission of all other lines and deal only with the Fe II spectrum (Fig [15). We fixed as many as possible Gaussian parameters, e.g. the ratio of [OIII] lines, or widths of the Balmer line components NLR, ILR and BLR (ILR - intermidiate-line region, see also Zhang 2011) The line parameters

\footnotetext{
${ }^{2}$ The atomic data was taken from the NIST atomic database: http://www.nist.gov
} 
inferred from our fits (width, shift, intensity relative to total $\mathrm{H} \beta$ ) are given in Table 10.

Kovačević et al. (2010) divided the Fe II emission into subgroups according to the lower level of the transition. We used the same criteria, thus we considered here 6 line groups: ${ }^{4} \mathrm{P}$, ${ }^{4} \mathrm{~F},{ }^{6} \mathrm{~S},{ }^{4} \mathrm{G},{ }^{2} \mathrm{H}$, and I Zw 1 line group. In Fig. 16 we plot the fluxes of all these line groups, and the total Fe II emission in the 4100-5600 , against the continuum flux at $5100 \AA$. We omitted the ${ }^{4} \mathrm{P}$ group, since below $4200 \AA$ the points are missing for more than $50 \%$ of considered spectra, and thus the flux measurements for this group are systematically lower. The total Fe II emission is correlating well with the continuum $\left(\mathrm{r} \sim 0.63, \mathrm{P}<10^{-10}\right)$. This correlation is slightly smaller than the one obtained for the measured Fe II in the wavelength region 5100-5470 $\AA$ (see Fig. 11). The $\mathrm{Fe}{ }^{4} \mathrm{G}$ line group consists of the transitions that contribute the most to the Fe II emission in 5100-5470 range, and for this line group we obtained practically the same correlation with the continuum variation as it was measured and presented in Fig. $11\left(\mathrm{r} \sim 0.74, \mathrm{P}<10^{-16}\right)$. A relatively good correlations $(\mathrm{r} \sim 0.50$, $\mathrm{P}<10^{-6}$ ) is obtained for ${ }^{4} \mathrm{G}$ group (lines located in the blue part of the Fe II shelf) and for the high-excitation energy group of I $\mathrm{Zw} 1\left(\mathrm{r} \sim 0.56, \mathrm{P}<10^{-8}\right)$, while the other two groups have no correlation at all: $\mathrm{Fe}^{2} \mathrm{H}(\mathrm{r} \sim-0.01$ and $\mathrm{P}=0.82)$ and $\mathrm{Fe}{ }^{6} \mathrm{~S}(\mathrm{r} \sim 0.14$ and $\mathrm{P}=0.18)$. In the case of $\mathrm{Fe}^{2} \mathrm{H}$ this could probably be due the very weak emission coming from these transitions, while in $\mathrm{Fe}{ }^{6} \mathrm{~S}$ it seems to be a real effect.

We compared the width of the Fe II lines to the widths of different $\mathrm{H} \beta$ components (Table 10). The average value of the Fe II lines is the same (within the error bars) to the average width value of the ILR component of the $\mathrm{H} \beta$ line (Fig. 17). This clearly supports the idea that the origin of the Fe II emission is more within the ILR than the BLR as stated before (see e.g. Marziani \& Sulentid 1993; Popović et al. 2004, 2009; Kovačević et al. 2010).

The quantity $\mathrm{R}_{\mathrm{Fe}}$, defined as the flux ratio of optical Fe II emission to $\mathrm{H} \beta$ line, is an important one in describing the EV1 parameter space (see Boroson \& Green 1992). Here 
the $\mathrm{H} \beta$ flux includes the contributions of all three components (narrow, intermidiate and broad), but still represents the behavior of the broad $\mathrm{H} \beta$ since the flux of the narrow component is expected to be constant. The variations of $\mathrm{R}_{\mathrm{Fe}}$ as a function of the blue continuum flux are plotted in Fig. 18. This plot shows a weak (statistically insignificant), but positive correlation between $\mathrm{R}_{\mathrm{Fe}}$ and continuum flux (the correlation coefficient $\mathrm{r} \sim 0.36$, and $\left.\mathrm{P}<10^{-3}\right)$

\section{Discussion}

\subsection{The structure of the line emitting region in Ark 564}

The permitted-line profiles of Ark 564 are Lorentzian like, that can be found in a group of AGNs with FWHM of broad lines smaller than $4000 \mathrm{~km} \mathrm{~s}^{-1}$ (Sulentic et al. 2009; Marziani et al. 2010; Sulentic et al. 2011). The problem is, that, such line profiles are not expected in the classical BLR. The Lorentzian-like line profile can be caused by the composition of the three Gaussian profiles (as it was shown in Kovačević et al. 2010),

where the contribution of the narrow component is significant. As e.g. Contini et al. (2003) roughly estimated the contribution of the BLR to the total $\mathrm{H} \beta$ line and to the permitted lines in the UV and found that $\mathrm{H} \beta_{\text {broad }} / \mathrm{H} \beta_{\text {narrow }}$ should range between 1 and 2 , that is not far from our estimation, that narrow component contributes to the total line flux with $\sim 20 \%$. Also, Rodríguez-Ardila et al. (2000) showed that the flux carried out by the narrow component of $\mathrm{H} \beta$ in a sample of seven NLS1s is, on average, $50 \%$ of the total line flux. Therefore, such high contribution of the narrow component to the total line flux can bring a small rate of variation in broad lines, and that the emission of the very broad component is very weak, i.e. that a relatively small fraction of the total flux in lines is coming from the BLR. 
The observed weak variation in the permitted lines of Ark 564 during a 10-year period is in agreement with a short term monitoring covering 2-year period given by Shemmer et al. (2001), they found no significant optical line variations. It is interesting that there is a weak correlation between the permitted lines and continuum variation. This, as well as the lack of correlation between $\mathrm{H} \alpha$ and $\mathrm{H} \beta$, may indicate different sources of ionization, as e.g. the shock wave ionization in addition to the photoionization. Moreover, five flare-like events (two prominent and three possible ones) are registered during the monitoring period, that confirm flare-like variability reported in Collier et al. (2001) and indicate burst events in the emission line regions. These may indicate some kind of explosions (in starburst regions) which can additionally affect the line and continuum emission. The small $[\mathrm{OIII}] / \mathrm{H} \beta_{\text {narrow }}$ ratio may also indicate a presence of starbursts in the center of Ark 564 (as it was noted for Mrk 493, see Popović et al. 2009).

Taking into account that it is very hard to properly decompose the narrow component (see discussion in Popović \& Kovačević 2011, in more details) from the broad one, it is hard to discuss about the geometry of the BLR of Ark 564. However, a lack of significant correlation between the $\mathrm{H} \alpha$ and $\mathrm{H} \beta$ flux variation, may indicate that there is a very stratified (in physical parameters, see Sec. 4.1) emitting region, where the $\mathbf{H} \beta$ emitting region tends to be more compact than the $\mathbf{H} \alpha$ one. This idea is supported by the detected differences in the FWHM and FWOI of $\mathbf{H} \alpha$ and $\mathbf{H} \beta$, and the absent correlation between the BD and continuum (Fig. 8). On the other hand, the quasi-simultaneous variations of $\mathbf{H} \alpha$ and $\mathbf{H} \beta$ blue and red wing (Fig. 6) and their good correlations (Fig. 9) indicate a predominantly circular motions in the BLR.

We calculated the CCF and found the delay of only a couple of days. Taking the 
$\mathbf{H} \beta$ width and lag (assuming that the $\mathbf{H} \beta$ lag corresponds to the dimension of the BLR) one can estimate the mass of the black hole of $\sim 1 \cdot 10^{6}$ Solar masses, that is in agreement with previous estimates by Shemmer et al. (2001); Collier et al. (2001); Pounds et al. (2001), and also well fit the hypothesis that NLS1s have lower black hole masses than typical Sy1s. However, one should take with caution this estimates, since there is no large correlation between the permitted lines and continuum variability. The CCF may indicate that the variability (perturbation) is coming from relatively small region, but it is interesting that it causes amplification of total line flux without significant change in line profiles (even during flare-like events). The permitted line profiles stay practically the same during the whole monitoring period (see Fig. 71).

\subsection{Fe II emission variability in Ark 564}

The variability behavior of the Fe II complex in Seyfert galaxies has been poorly understood (see Collin \& Joly 2000). As e.g. Kollatschny et al. (2001) reported that in Mrk 110 the permitted optical Fe II complex remained constant within $10 \%$ error over 10 years, while the forbidden [Fe X] $\lambda 6375$ line was variable. Similarly, in the Seyfert 1 galaxy NGC 5548 no significant variations of the optical Fe II blends (less than 20\%) were detected (Dietrich et al. 1993). However, the opposite result was reported in a long term optical variability watch program on Seyfert 1 galaxy NGC 7603 over a period of nearly 20 years (Kollatschny et al. 2000). This object displayed remarkable variability in the Fe II feature, with amplitudes of the same order as for the $\mathrm{H} \alpha$ and He I lines. Giannuzzo \& Stripe (1996) found that, out of 12 NLS1s, at least 4 of them presented a significant variability of the Fe II complex with percentage variations larger than 30\%. In addition, considerable variations of the Fe II emission (larger than 50\%) were reported in two Seyfert 1 galaxies: 
Akn 120 and Fairall 9 (Kollatschny et al. 1981; Kollatschny \& Fricke 1985). On the other hand Kuehn et al. (2008) performed a reverberation analysis of the strong, variable optical Fe II emission bands in the spectrum of Ark 120 and they were unable to measure a clear reverberation lag for these Fe II lines on any timescale. They concluded that the optical Fe II emission does not come from a photoionization-powered region similar in size to the $\mathrm{H} \beta$ emitting region. Our results confirm this since for different groups there are different correlation with the continuum and in some groups (as e.g. ${ }^{6} S$ ) there is no correlation at all (see discussion below).

The most interesting is that the Fe II variation (at least in the red part of Fe II shelfs) in Ark 564 is closely following the variations in the continuum. Similar result was obtained in the case of NGC 4051, an NLS1 galaxy, where the variability of the optical Fe II emission also followed the continuum variability (Wang et al. 2005).

We investigate the time variability of several Fe II multiplets in Ark 564. An interesting result is that there are different levels of correlations between the emission of Fe II line groups and continuum flux. It seems that the level of Fe II flux variability depends on the type of transition. For example, we found the good correlation for ${ }^{4} \mathrm{G}$ and ${ }^{4} \mathrm{~F}$ groups which mainly contribute to the blue and red Fe II features around $\mathrm{H} \beta$, and practically no significant correlation between ${ }^{2} \mathrm{H}$ and ${ }^{6} \mathrm{~S}$ group 3 and continuum (Fig. 13). The emission from these two groups seem to be variable, but there is no respons to the continuum variability. This also may indicate that the Fe II emission region in Ark 564 is stratified. On the other hand, the width of Fe II lines follow the width of the ILR component, that is in a good agreement with results reported in previous work (Marziani \& Sulentid 1993;

\footnotetext{
${ }^{3}$ Note that the ${ }^{6} \mathrm{~S}$ FeII group may be affected by the [OIII $] 5007$ line, therefore the lack of a correlation for this component might be simply due to measurement bias.
} 
Popović et al. 2004, 2009; Kovačević et al. 2010)

We found a positive (yet, statistically not significant) trend of $R_{\mathrm{Fe}}$ with the blue continuum. Wang et al. (2005) found the similar positive trend for the galaxy NGC 4051, in opposition to the negative trend observed in NGC 7603 (reported by Wang et al. 2005, on the data of Kollatschny et al. 2000). They argued, by comparing the variability behaviors of different objects, that the objects with positive correlations have narrow $\mathrm{H} \beta$ lines and consequently are classified as NLS1s. While the remaining two sources with negative correlations have relatively broad $\mathrm{H} \beta$ profiles. They interpreted that the dichotomy in variability behavior of $\mathrm{R}_{\mathrm{Fe}}$ is due to the different physical conditions governing the variability of the optical Fe II emission. Our result is consistent with their findings supporting their idea that in case of NLS1 we have that the bulk excitation of the optical Fe II lines is due to collisional excitation in a high density optically thick cloud illuminated and heated mainly by X-rays photons (see Wang et al. 2005, and references therein).

\section{Conclusion}

In this paper, we analyzed the long spectral variability of NLS1 galaxy Ark 564, observed in the 11-year period from 1999 to 2010. We performed a detail analysis of optical spectra covering the continuum flux at $5100 \AA$ and $6200 \AA$ and $\mathrm{H} \beta, \mathrm{H} \alpha$ and Fe II lines. Here we briefly outline our conclusion:

1) In Ark 564 during the monitoring period (1999-2010) the mean continuum and lines fluxes decreased for $\sim 20 \%-30 \%$ (see Fig. 5) from the beginning (1999) to the end of the monitoring (2010). The total flux of Fe II evidently increases with the continuum flux.

2) We registered five flare-like events (two prominent and three possible) lasting $\sim 1-3$ days, when fluxes in continuum and lines changed for $\sim 20 \%$ (continuum and Fe II emission) 
and $\sim 10 \%$ for Balmer lines.

3) The flux-flux correlations between the continuum and lines are weak, where the correlation between the Fe II lines (in the red shelf of the Fe II) and continuum is slightly higher (and more significant) than between the Balmer lines and continuum. There is almost lack of correlation between the $\mathrm{H} \alpha$ and $\mathrm{H} \beta$ line fluxes. Such behavior indicate very complex physical processes in the line forming region, i.e. beside the photoionization some additional physical processes may be present.

4) We roughly estimated a lag of 2-6 days, but with large errorbars. Taking that the photoionization is probably not the only source of line excitation, the obtained results should be taken with caution.

5) We investigated in detail, the Fe II emission variability. We divided the Fe II emission in six groups according to the atomic transitions. We found that correlation between the continuum flux and emission of groups depends on the type of transition, i.e. in some case there is relatively good correlation level between the Fe II group emission $\left({ }^{4} \mathrm{G}\right.$, ${ }^{4} \mathrm{~F}$ group), but for ${ }^{2} \mathrm{H}$ and ${ }^{6} \mathrm{~S}$ there is no correlation at all.

6) The Gaussian multicomponent analysis indicates that the emission of the Fe II lines is probably coming from the intermidiate line region, having velocities around $1500 \mathrm{~km} / \mathrm{s}$.

The spectral variability of Ark 564 seems to be complex and different from the one observed in BLS1 (see e.g. Shapovalova et al. 2009). The observed flare-like events, the small (or even lack of) correlation between $\mathrm{H} \alpha$ and $\mathrm{H} \beta$ fluxes, the different correlation degree for Fe II group emission and continuum light level, may indicate complex physics in the emitting regions, as e.g. there may be, beside the AGN, contribution of star explosions and internal shock waves.

We thank the anonymous referee for very useful comments and suggestions. This 
work was supported by RFBR research grants N00-02-16272, N03-02-17123, 06-02-16843, N09-02-01136, N12-02-01237a (Russia), CONACYT research grants 39560-F, 54480, 151494 and PAPIIT-UNAM research grant IN111610 (México), and the projects "Astrophysical Spectroscopy of Extragalactic Objects" (176001) supported by the Ministry of Education and Science of the Republic of Serbia. L. Č. P, W. K., D. I. and J. K. are grateful to the Alexander von Humboldt foundation for support in the frame of program "Research Group Linkage". 


\section{REFERENCES}

Alexander, T. 1997, Astronomical Time Series, ed. D. Maoz, A. Sternberg, \& E. M. Leibowitz (Dordrecht: Kluwer), 163

Bischoff, K., \& Kollatschny, W. 1999, A\&A, 345, 49B

Boller, Th., Brandt, W. N., Fink, H. 1996, A\&A, 305, 53

Boroson, T. A., \& Green, R. F. 1992, ApJS, 80, 109

Chiho, M., Karen M. L., Herman L. M. 2004, ApJ, 603, 456

Crenshaw, D. M., Kraemer, S. B., Boggess, A., Maran, S. P., Mushotzky, R. F., Wu, C.-C. 1999, ApJ, 516, 750

Crenshaw, D. M., Kraemer, S. B., Turner, T. J., et al. 2002, ApJ, 566, 187

Cruise, A. M., \& Dodds, P. M. 1985, MNRAS, 215, 417

Collier, S., Crenshaw, D. M., Peterson, B. M. et al. 2001, 561, 146

Collin, S., Joly, M. 2000, NewAR, 44, 531

Contini, M., Rodríguez-Ardila, A., Viegas, S. M. 2003, A\&A, 408, 101

De Vaucouleurs, G., De Vaucouleurs, A., Corwin, H. G. Jr., et al. 1991, in Third Reference Catalogue of Bright Galaxies (New York: Springer-Verlag)

Dietrich, M., Kollatschny, W., Peterson, B. M., et al. 1993, ApJ, 408, 416

Hufnagel, B. R., \& Bregman, J. N. 1992, ApJ, 386, 473

Hughes, P. A., Aller, H. D., Aller, M. F. 1992, ApJ, 396, 469

Giannuzzo, E. M., Stirpe, G. M. 1996, A\&A, 314, 419 
Ilić, D., Popović, L. Č., La Mura, G., Ciroi, S., Rafanelli, P. 2012, A\&A, accepted (eprint arXiv:1205.3950)

Kollatschny, W., Bischoff, K., Dietrich, M. 2000, A\&A, 361, 901

Kollatschny, W., Bischoff, K., Robinson, E. L., Welsh, W. F., Hill, G. J. 2001, A\&A, 379, 125

Kollatschny, W., Fricke, K. J. 1985, A\&A, 146, L11

Kollatschny, W., Schleicher, H., Fricke, K. J., Yorke, H. W. 1981, A\&A, 104, 198

Kovačević, J., Popović, L. Č., Dimitrijević, M. S. 2010, ApJS, 189, 15

Kuehn, C. A., Baldwin, J. A., Peterson, B. M., Korista, K. T. 2008, ApJ, 673, 69

Kuraszkiewicz, J. K., Green, P. J., Forster, K., Aldcroft, T. L., Evans, I. N., Koratkar, A. 2002, ApJS, 143, 257

Leighly, K. M. 1999a, ApJS, 125, 297

Leighly, K. M. 1999b, ApJS, 125, 317

Leighly, K. M. 2000, NewAR, 44, 395

Marziani, P., Sulentic, J. W. 1993, ApJ, 558, 553

Marziani, P., Sulentic, J. W., Negrete, C. A., Dultzin, D., Zamfir, S., Bachev, R. 2010, MNRAS, 409, 1033

O’Brien P.T., Dietrich, M., Leighly, K et al. 1998, ApJ, 509, 163

Osterbrock, D. E., Pogge, R. W. 1985, ApJ, 297, 166 
Panessa, F., de Rosa, A., Bassani, L., Bazzano, A., Bird, A., Landi, R., Malizia, A., Miniutti, G., Molina, M., Ubertini, P. 2011, MNRAS, 417, 2426

Peterson B. M., 1993, PASP105, 207

Peterson, B. M., Barth, A. J., Berlind, P. et al. 1999, ApJ, 510, 659

Peterson, B. M., Berlind, P., Bertram, R. et al. 1994, ApJ, 425, 622

Peterson, B. M., Berlind, P., Bertram, R. et al. 2002, ApJ. 581, 197

Peterson, B. M., \& Collins, G. W., II 1983, ApJ, 270, 71

Peterson, B. M., Pogge, R.W., Wanders, I., Smith, S. M., Romanishin, W. 1995, PASP, 107,579

Popović, L. Č., Kovačević, J. 2011, ApJ, 738, 68

Popovi, L. Č., Mediavilla, E., Bon, E., Ilić, D. 2004, A\&A, 423, 909

Popović, L. Č., Smirnova, A., Kovačević, J., Moiseev, A., Afanasiev, V. 2009, AJ, 137, 3548

Pounds, K., Edelson, R., Markowitz, A., Vaughan, S. 2001, ApJ, 550, 15

Rodríguez-Ardila, A., Pastoriza, M. G., Binette, L., \& Donzelli, C. J. 2000, ApJ, 538, 581

Shapovalova, A. I., Doroshenko, V. T., Bochkarev, N. G. et al. 2004, A\&A, 422, 925

Shapovalova, A. I., Popović, L. Č., Collin, S., Burenkov, A. N., Chavushyan, V. H., Bochkarev, N. G., Benítez, E., Dultzin, D., Kovačević, A., Borisov, N., Carrasco, L., León-Tavares, J., Mercado, A., Valdes, J. R., Vlasuyk, V. V., Zhdanova, V. E. 2008, A\&A, 486, 99

Shapovalova, A. I., Popović, L. Č., Bochkarev, N. G., Burenkov, A. N., Chavushyan, V. H., Collin, S., Doroshenko, V. T., Ilić, D., Kovačević, A. 2009, NewAR, 53, 191. 
Shapovalova, A. I., Popović, L. Č., Chavushyan, V. H., et al. 2010, A\&A517, id.A42

Shemmer, O., Romano, P., Bertram, R. et al. 2001, ApJ, 561, 162

Smith, R. A. N.; Page, M. J.; Branduardi-Raymont, G. 2008, ApJ, 490, 103

Sulentic, J. W., Bachev, R., Marziani, P., Negrete, C. A., Dultzin, D. 2007, ApJ, 666, 757

Sulentic, J., Marziani, P., Zamfir, S. 2009, NewAR, 53, 198

Sulentic, J., Marziani, P., Zamfir, S. 2011, BaltA, 20, 427

Turner, T. J., Romano, P., George, I. M., Edelson, R., Collier, S. J., Mathur, S., Peterson, B. M. 2001, ApJ, 561, 131

Van Groningen, E. \& Wanders, I. 1992, PASP, 104, 700

Wang, J., Wei, J. Y., He, X. T.2005, A\&A, 436, 417

Zhang, X.-G. 2005, ApJ, 741, 104 


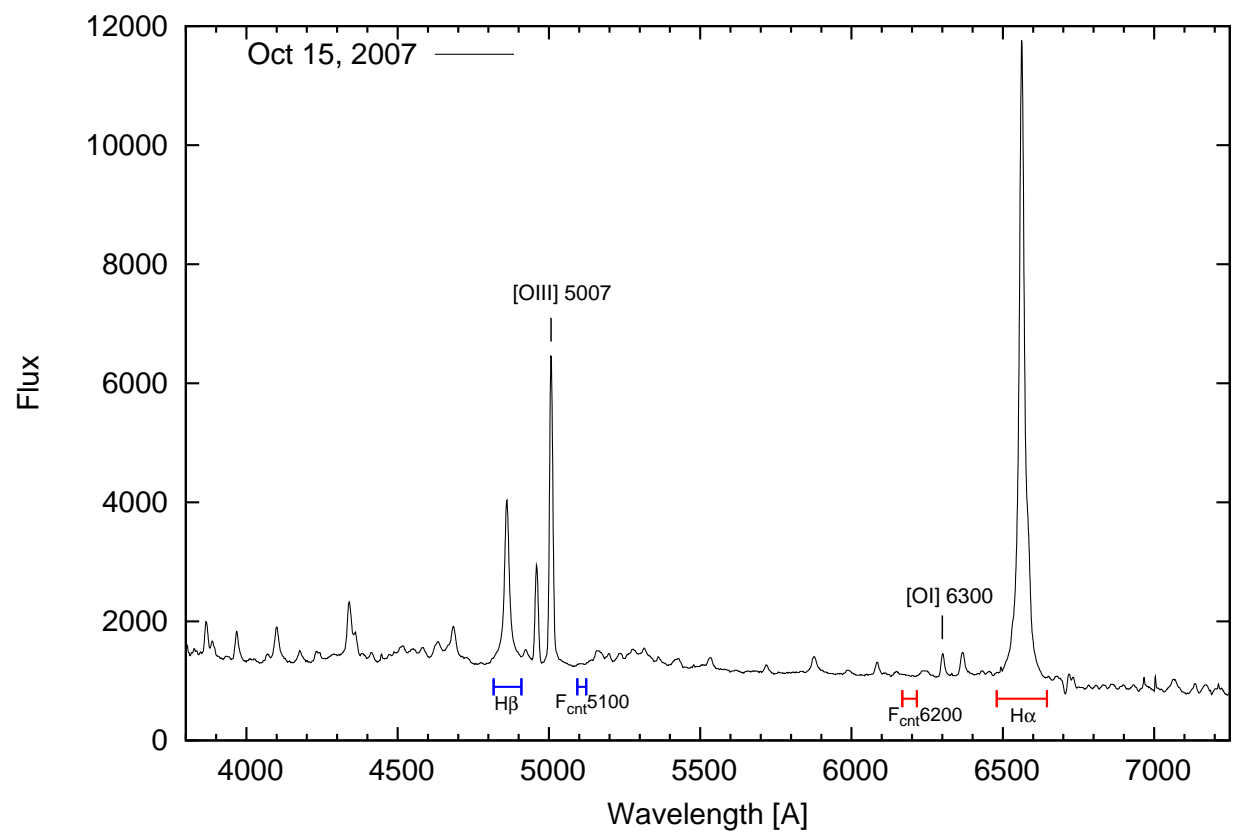

Fig. 1.- An example of the total optical spectrum of Ark 564. The windows for $\mathrm{H} \beta, \mathrm{H} \alpha$, blue and red continuum measurements are marked. 


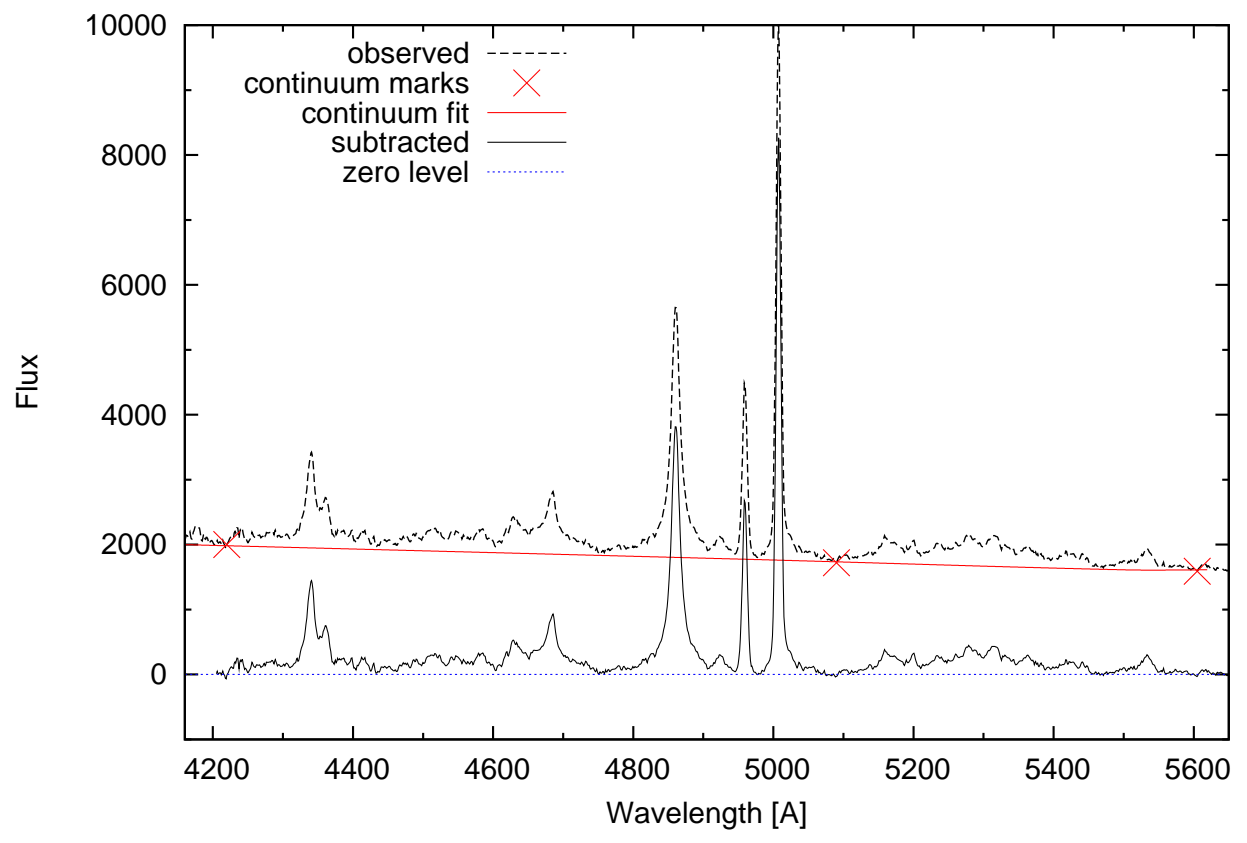

Fig. 2.- Underlying continuum subtraction in the $\mathrm{H} \beta$ region needed for accurate Fe II measurements. 

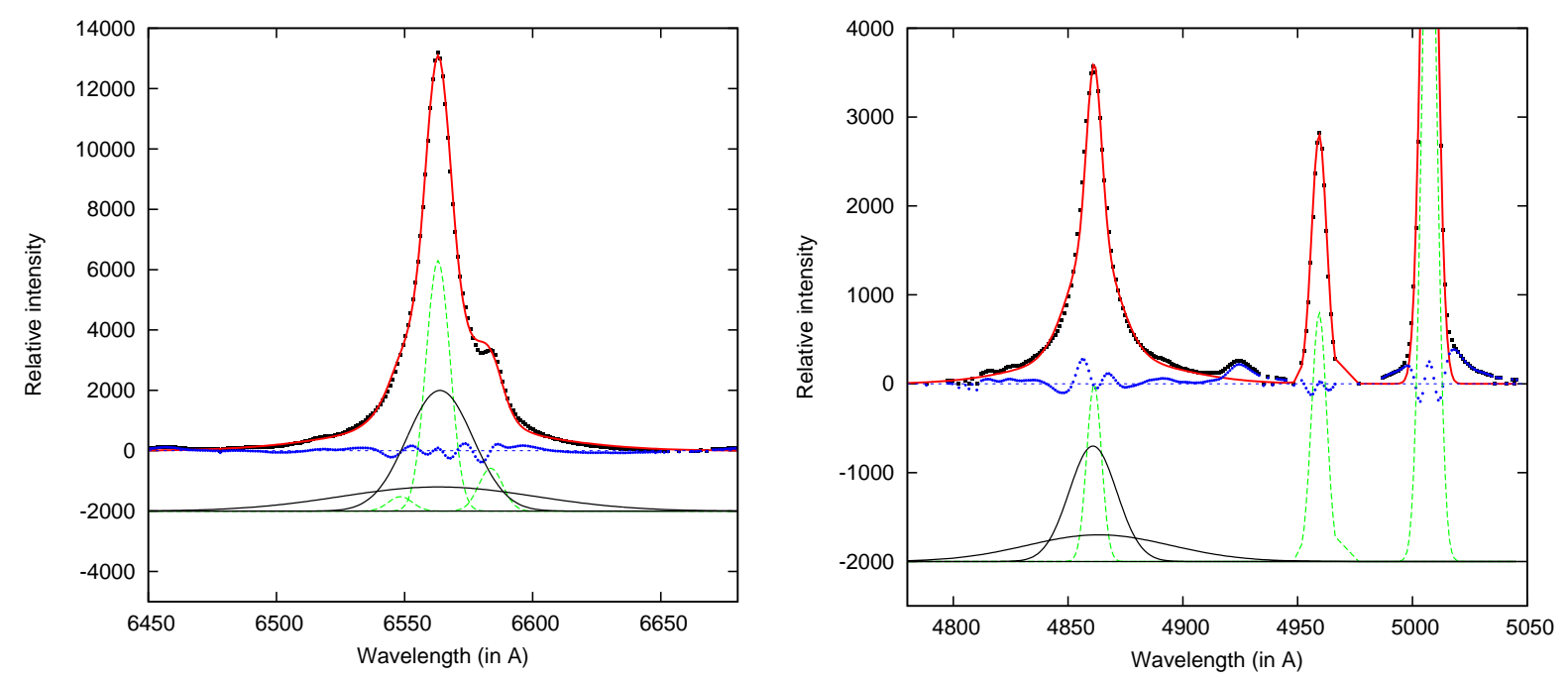

Fig. 3.- The best Gaussian fitting (solid line) of the mean $\mathrm{H} \alpha$ (left) and $\mathrm{H} \beta$ (right) line profiles (dotted line) with a sum of Gaussians. The broad components are fitted with two Gaussian (solid lines) and the narrow lines with one (dashed lines). The line residuals are also given below the observed spectra. In the region of the $\mathrm{H} \beta$ the Fe II contribution is not subtracted. 


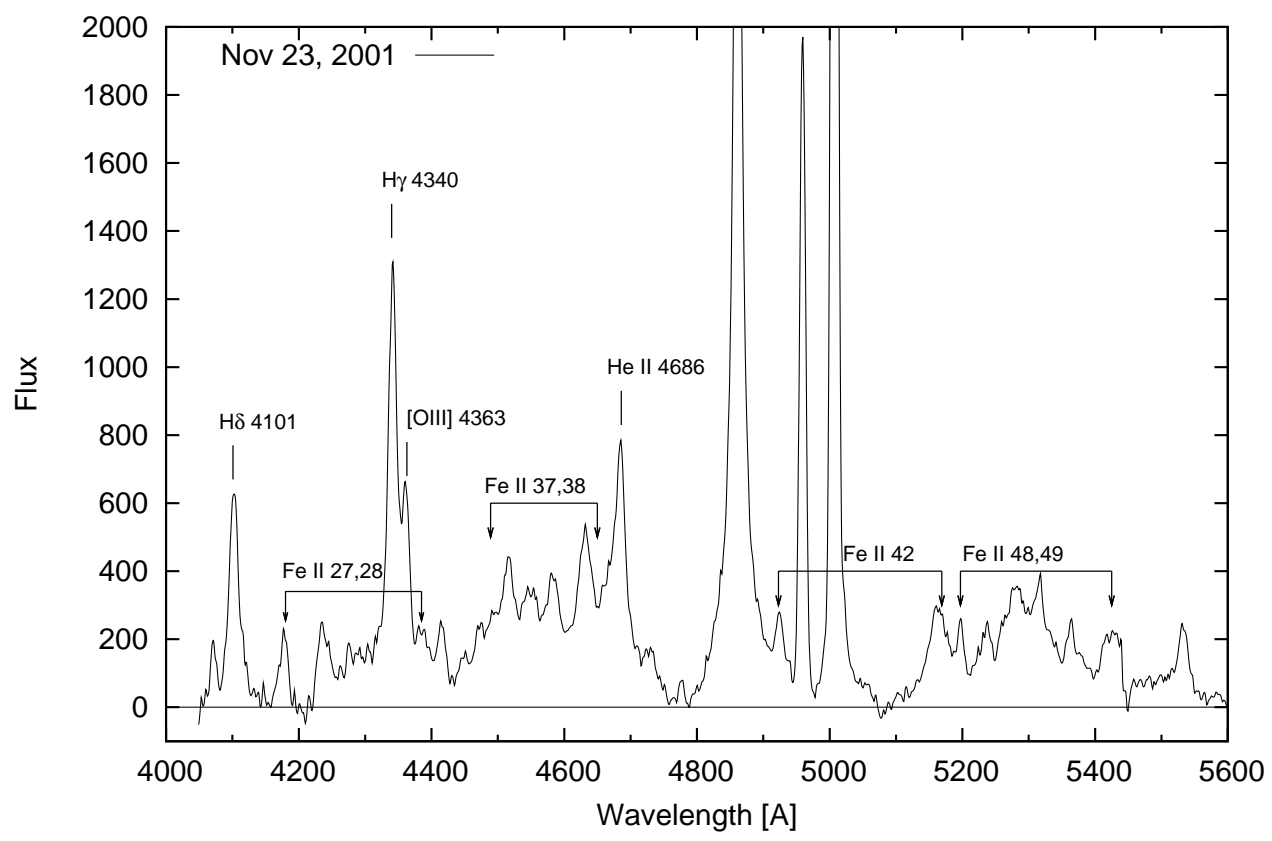

Fig. 4. - The Fe II emission around the $\mathrm{H} \beta$ line for Ark 564 . 


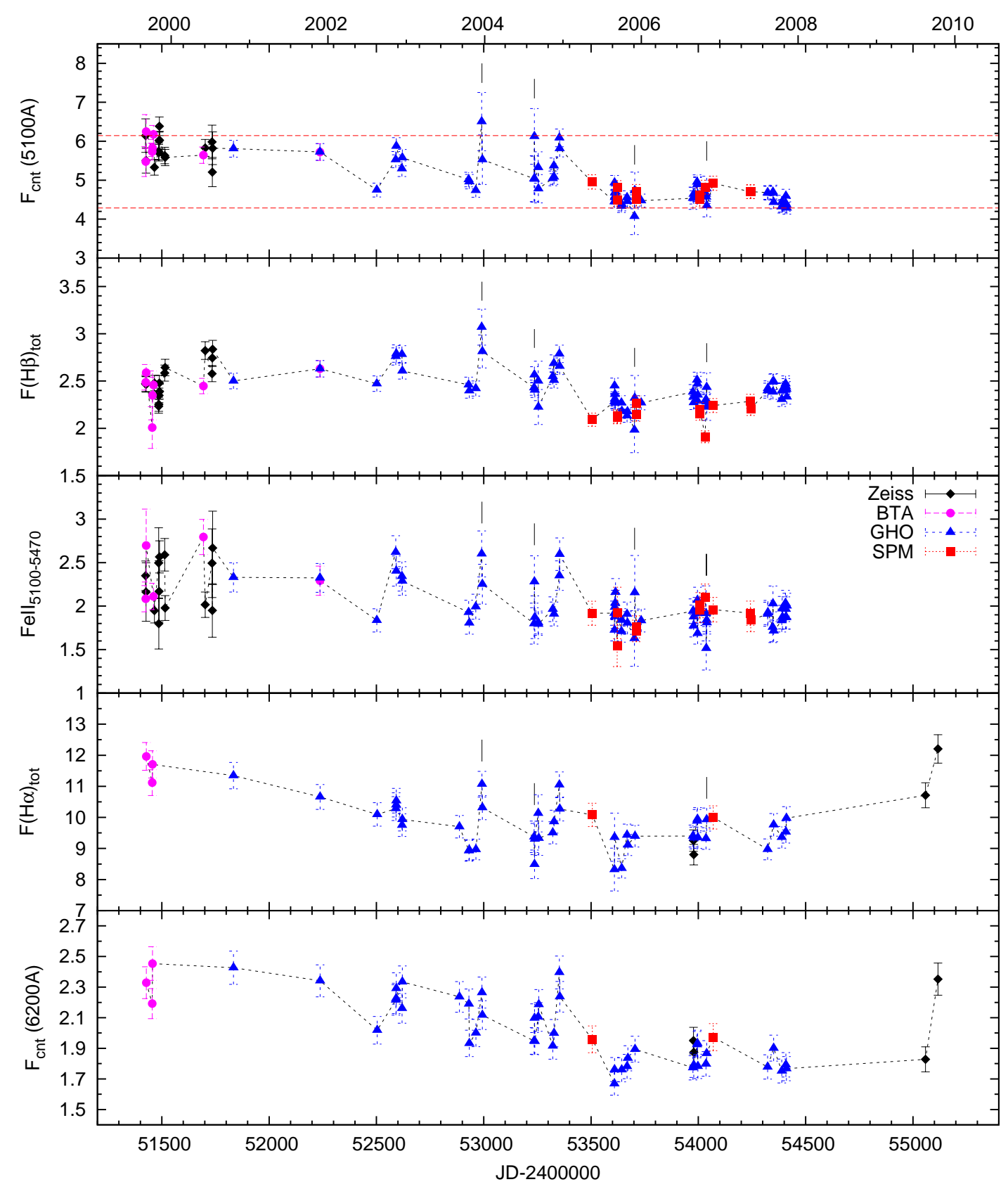

Fig. 5. - Light curves (from top to the bottom) of the continuum at $5100 \AA \mathrm{H} \beta$, Fe II, H $\alpha$ and the continuum at $6200 \AA$. Data obtained with different telescopes are marked with different symbols: diamonds - 6m BTA, circles - 1m Zeiss, triangles - 2.1m GHO, squares - 2.1m SPM. The flares are marked on the upper four plot (see Table 6), while in the blue continuum plot (first upper plot) the dashed lines represent the first and last observed continuum flux to show the decrease of the continuum flux during the monitoring campaign. Continuum fluxes are given in units $10^{-15} \mathrm{erg} \mathrm{cm}^{-2} \mathrm{~s}^{-1} \AA^{-1}$ and line fluxes in 

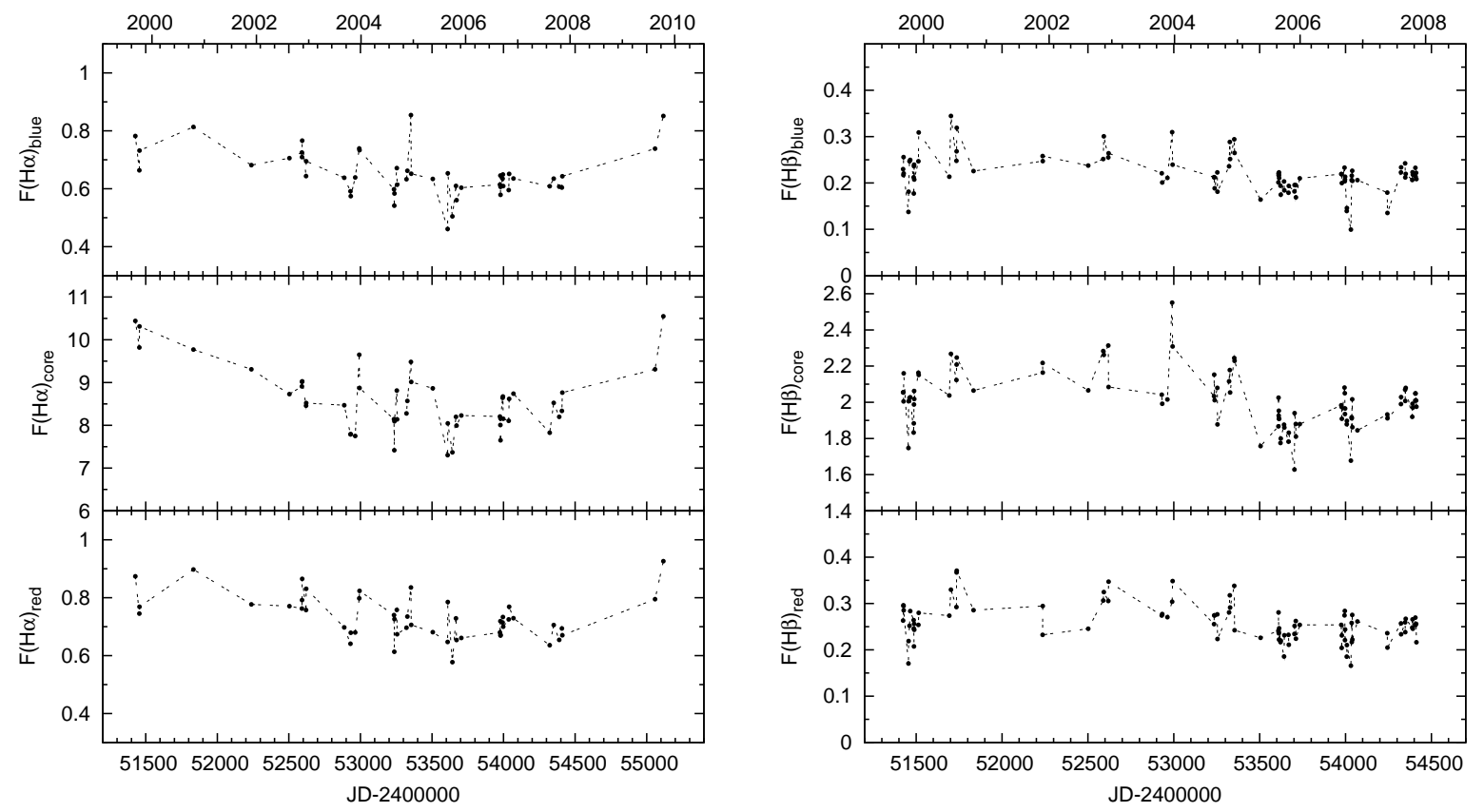

Fig. 6. - Light curves of the $\mathrm{H} \alpha$ (left) and $\mathrm{H} \beta$ (right) line-segments (from top to bottom: blue, core, and red line parts). 

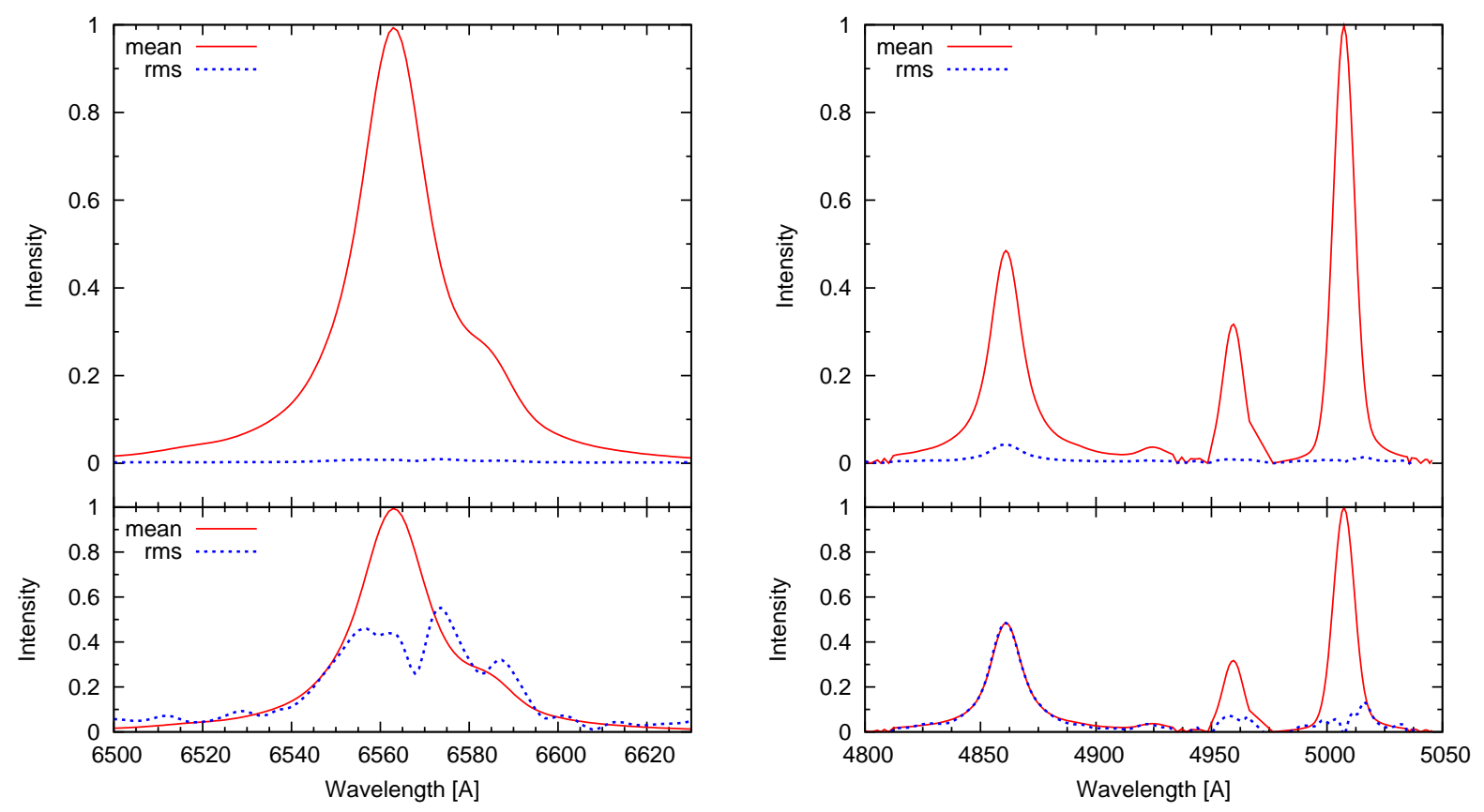

Fig. 7.- The mean and rms spectra of $\mathrm{H} \alpha$ (left) and $\mathrm{H} \beta$ (right) after calibrating the spectra to the same spectral resolution. Below plots show the normalized mean and rms spectra arbitrary scaled for comparison. 

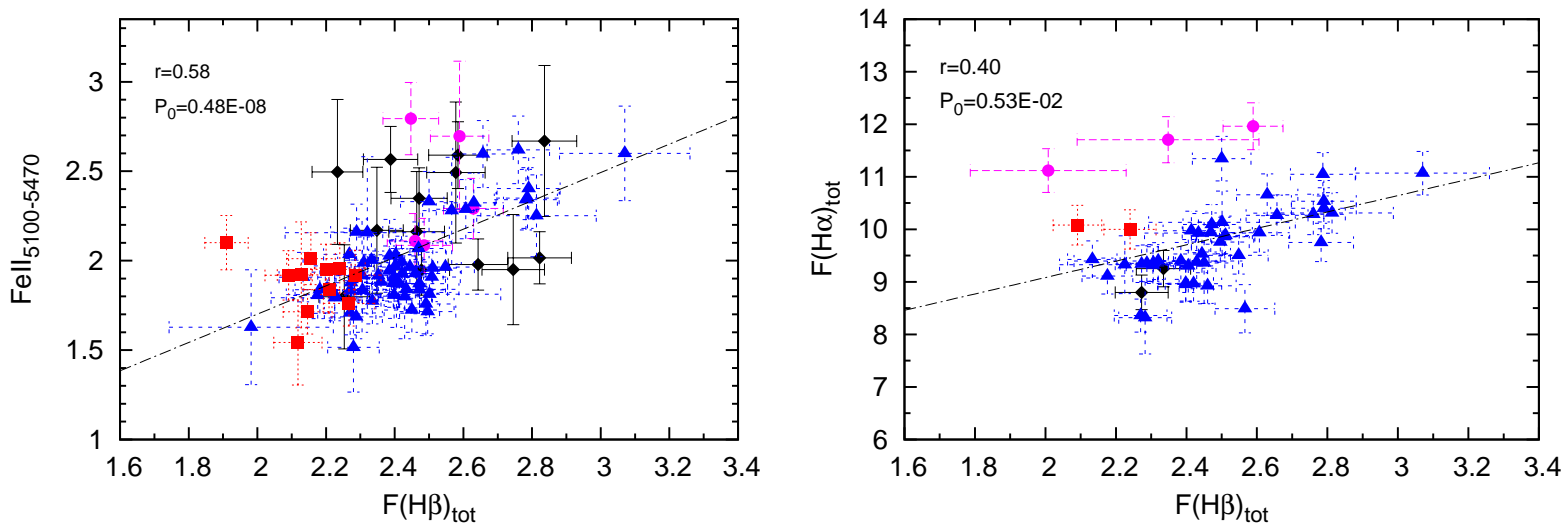

Fig. 8.- The Fe II red shelf (left) and $\mathrm{H} \alpha$ (right) line fluxes as a function of the $\mathrm{H} \beta$ line flux. The correlation coefficient and the corresponding P-value are given in the upper left corner. The notation is the same as in Fig. 5, 

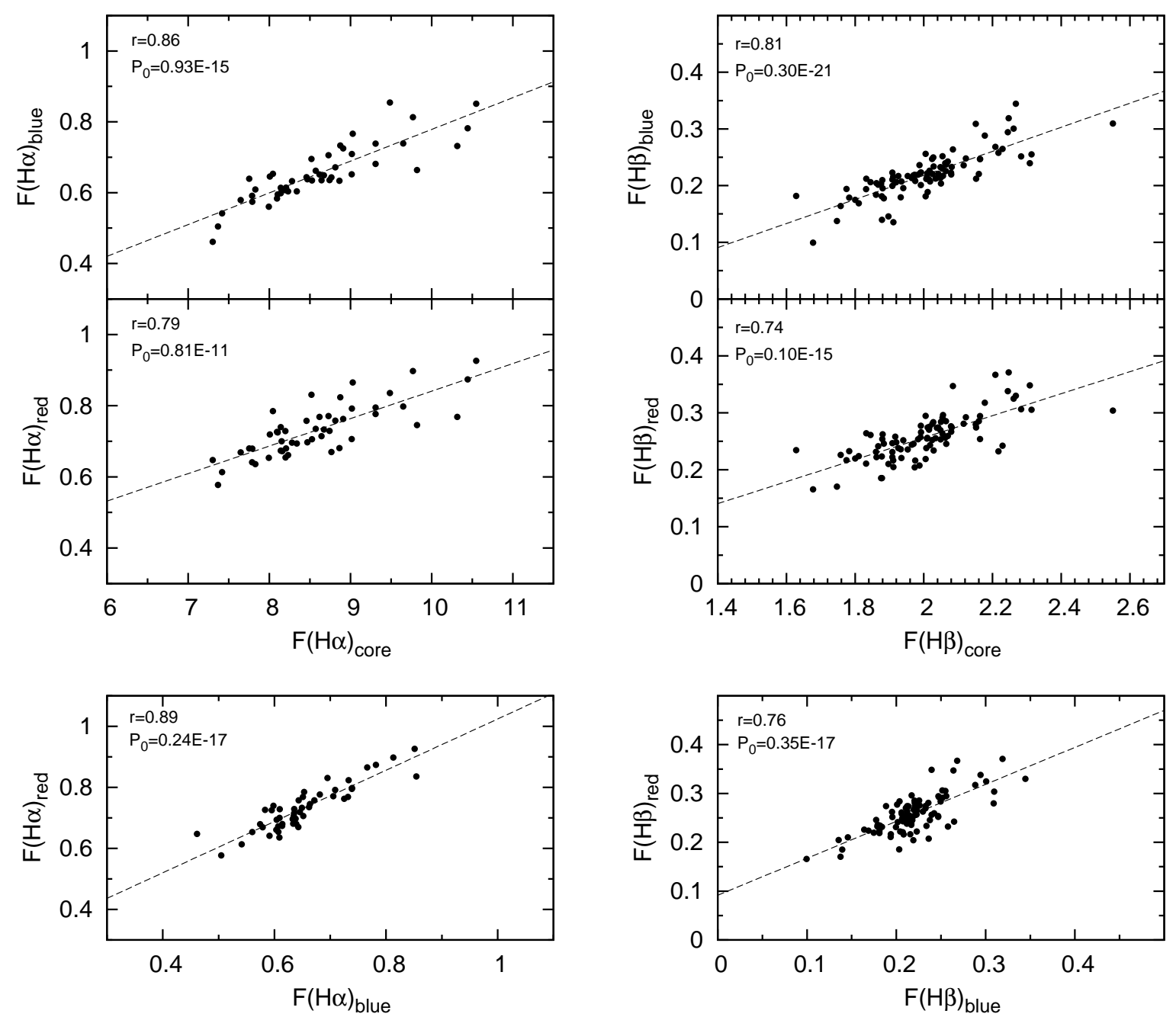

Fig. 9.- $\mathrm{H} \alpha$ and $\mathrm{H} \beta$ line-wing fluxes (blue, red) vs. line-core flux (upper panels), and red vs. blue-wing (bottom panel). The correlation coefficient and the corresponding P-value are given in the upper left corner. 

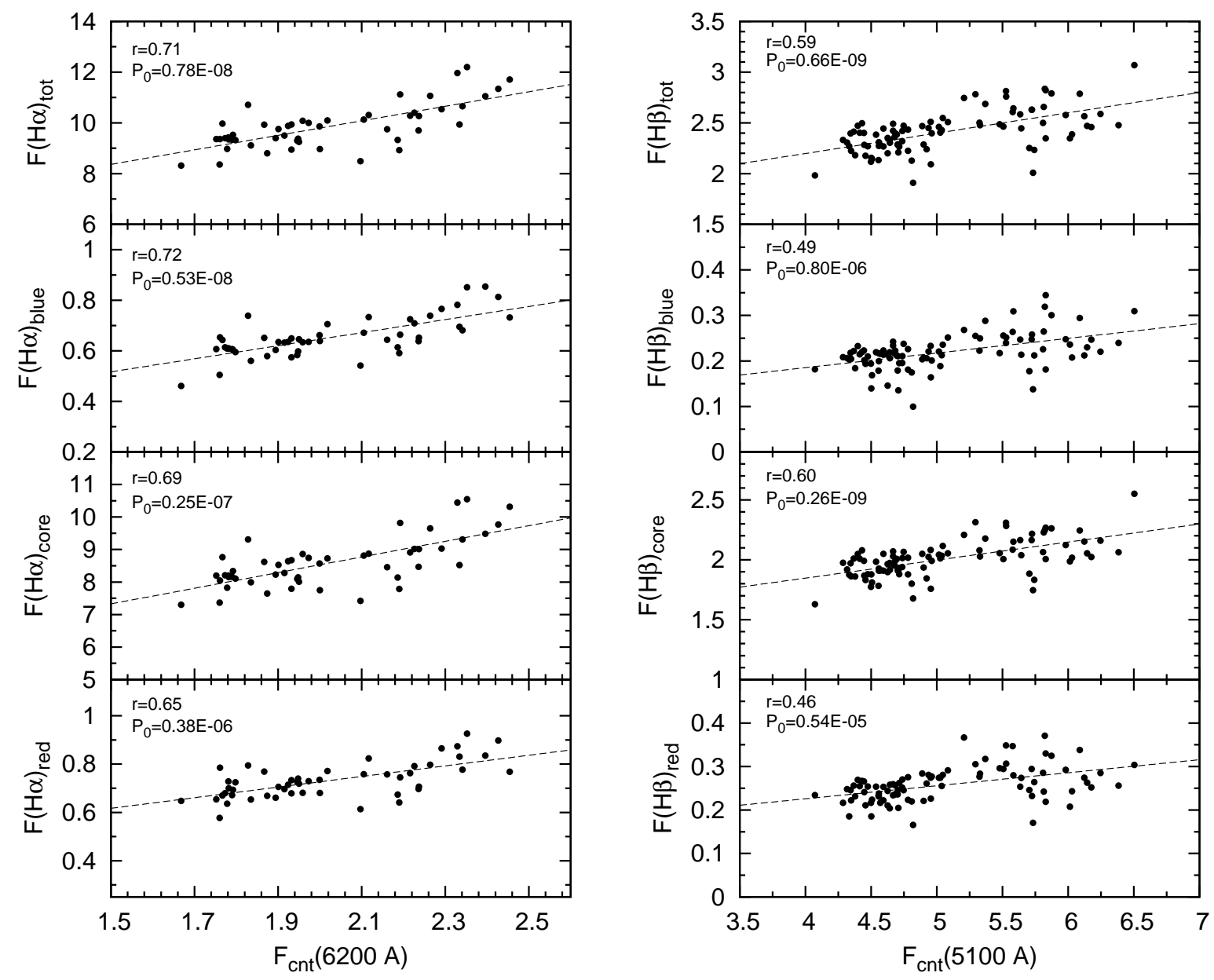

Fig. 10.- $\mathrm{H} \alpha$ and $\mathrm{H} \beta$ line and line-segment fluxes (blue, core and red) vs. continuum flux at 6300 and 5100, respectively. The correlation coefficient and the corresponding P-value are given in the upper left corner. 


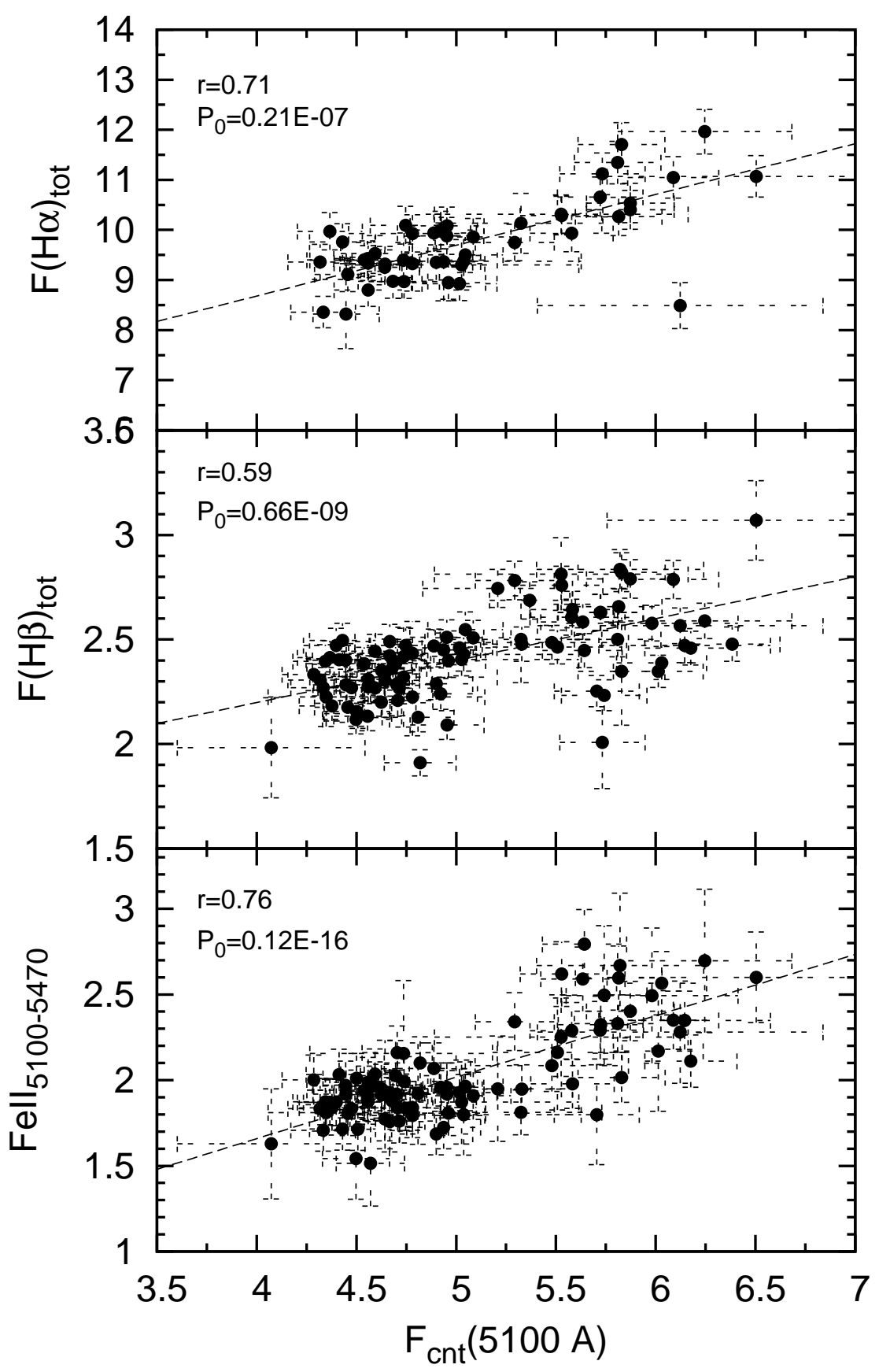

Fig. 11.- $\mathrm{H} \alpha$ (upper), $\mathrm{H} \beta$ (middle), Fe II (bottom) emission against the continuum flux at 5100. The correlation coefficient and the corresponding P-value are given in the upper left corner. 


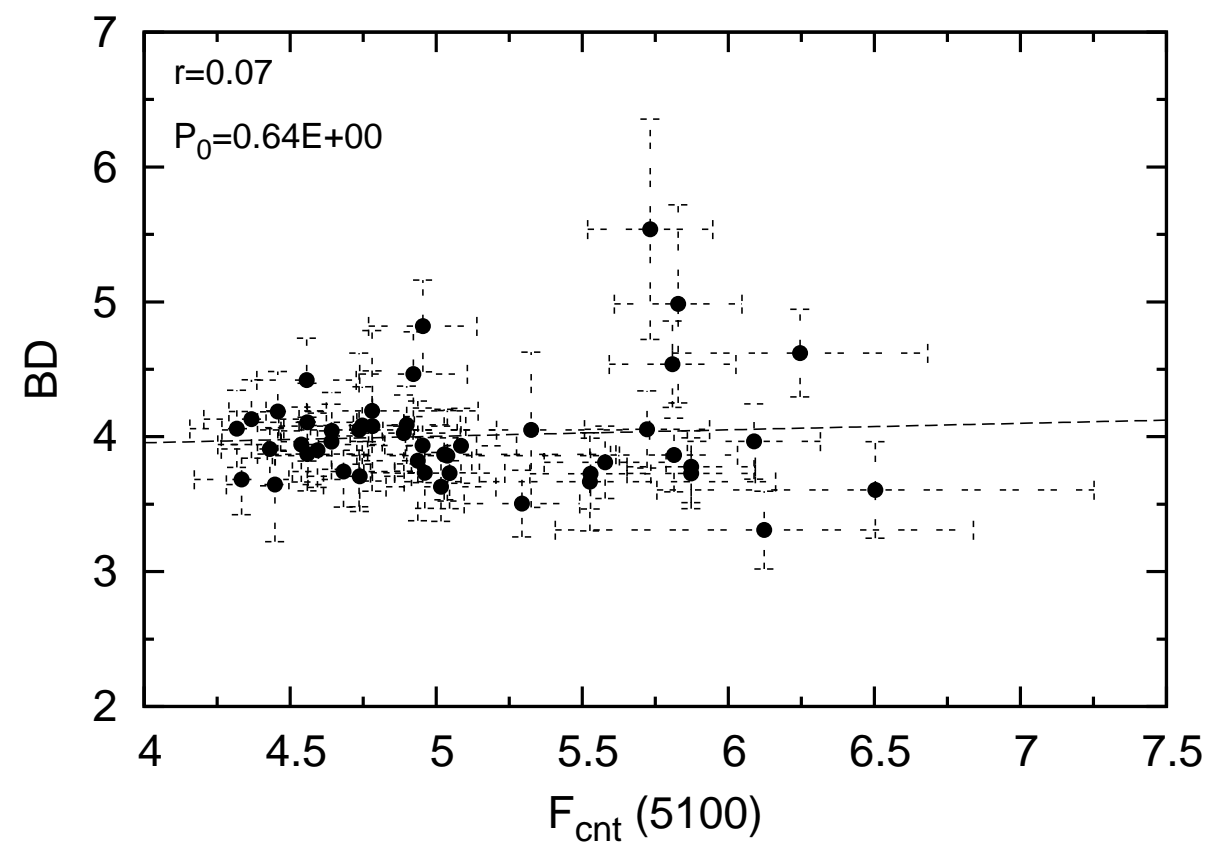

Fig. 12.- Balmer decrement vs. continuum flux at $5100 \AA$. The correlation coefficient and the corresponding $\mathrm{P}$-value are given in the upper left corner. 


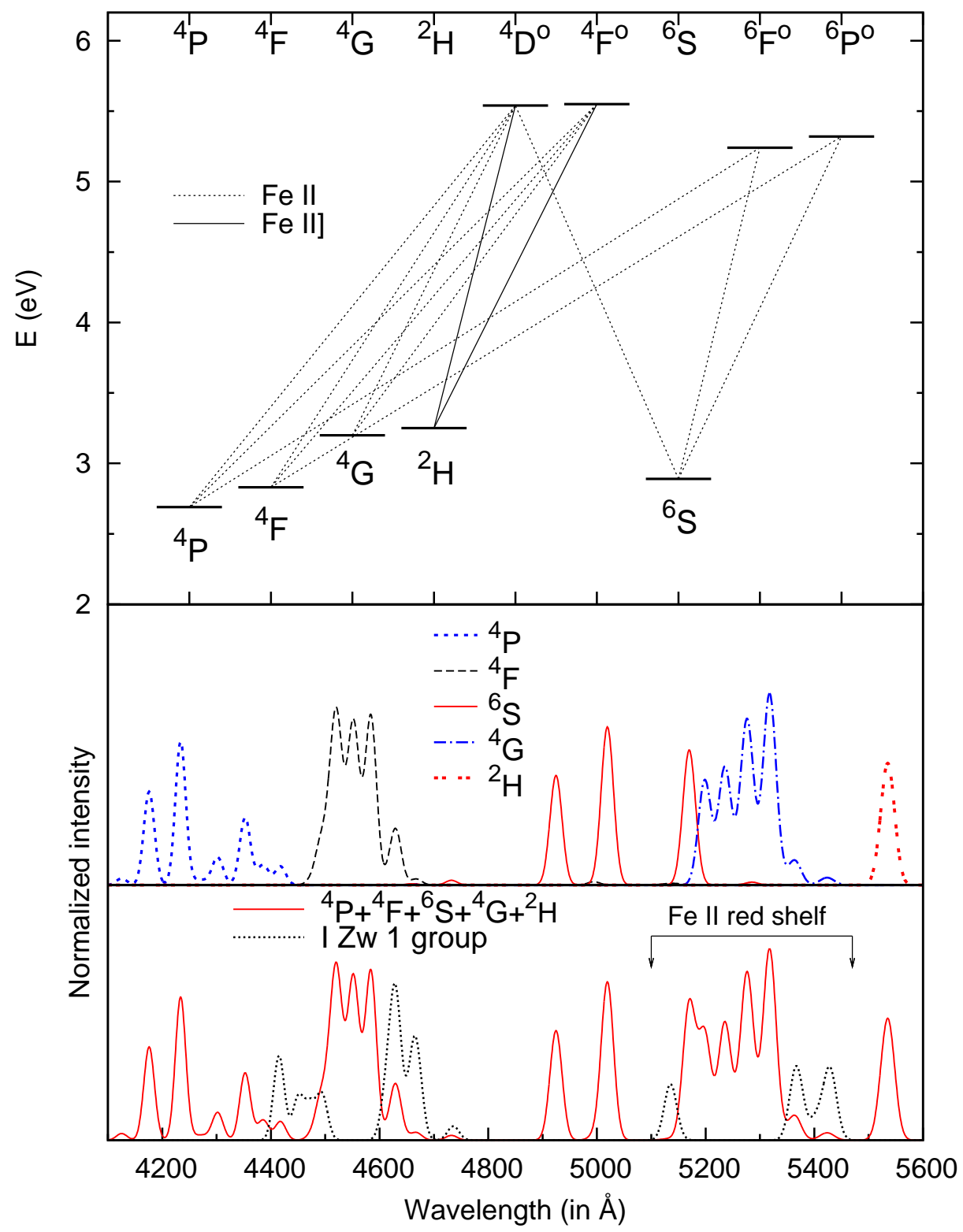

Fig. 13.- The simplified Grotrian diagram showing the strongest Fe II transitions in the $\lambda \lambda$ 4100-5600 $\AA$ region (top). Lines are separated into five groups according to the lower level of transition (middle): P (dotted line), F (dashed line), S (solid line), G (dash - dotted line), and $\mathrm{H}$ (two-dashed line). Bottom: the lines from the three line groups (solid line) and additional lines taken from I Zw 1 (see Kovačević et al. 2010), represented with dots. The measured red shelf region is also noted (Table 4). 


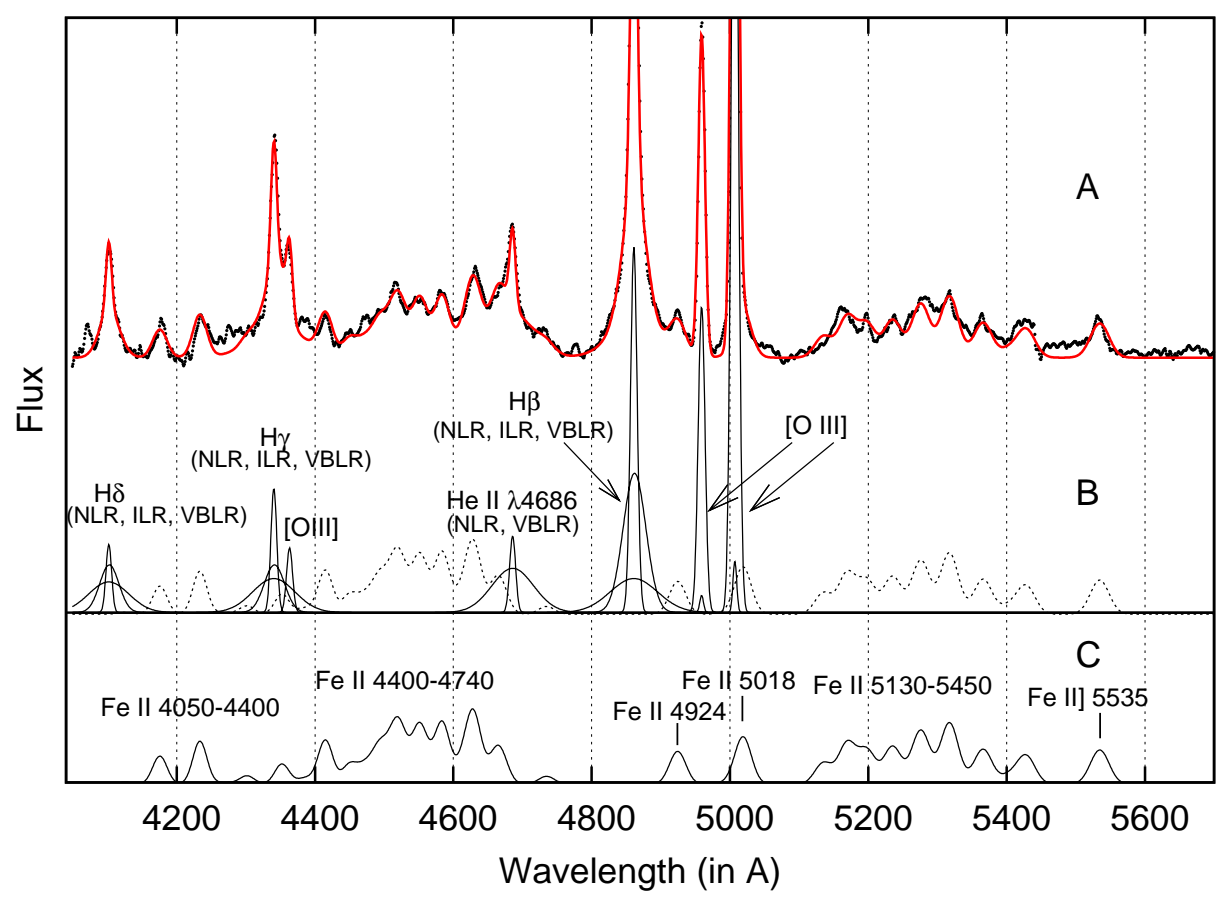

Fig. 14. - An example of the best fit (date Nov 23, 2001) of the $\lambda \lambda 4000-5600 \AA$ region: (A) the observed spectra (dots) and the best fit (solid line). (B) $\mathrm{H} \beta, \mathrm{H} \gamma$, and $\mathrm{H} \delta$ fit with the sum of three Gaussians representing emission from the NLR, ILR and BLR. The [O III] $\lambda \lambda 4959$, $5007 \AA$ lines are fit with two Gaussians for each line of the doublet and He II $\lambda 4686 \AA$ is fit with one broad and one narrow Gaussian. The Fe II template is denoted with a dotted line, and also represented separately in panel $(\mathrm{C})$. 


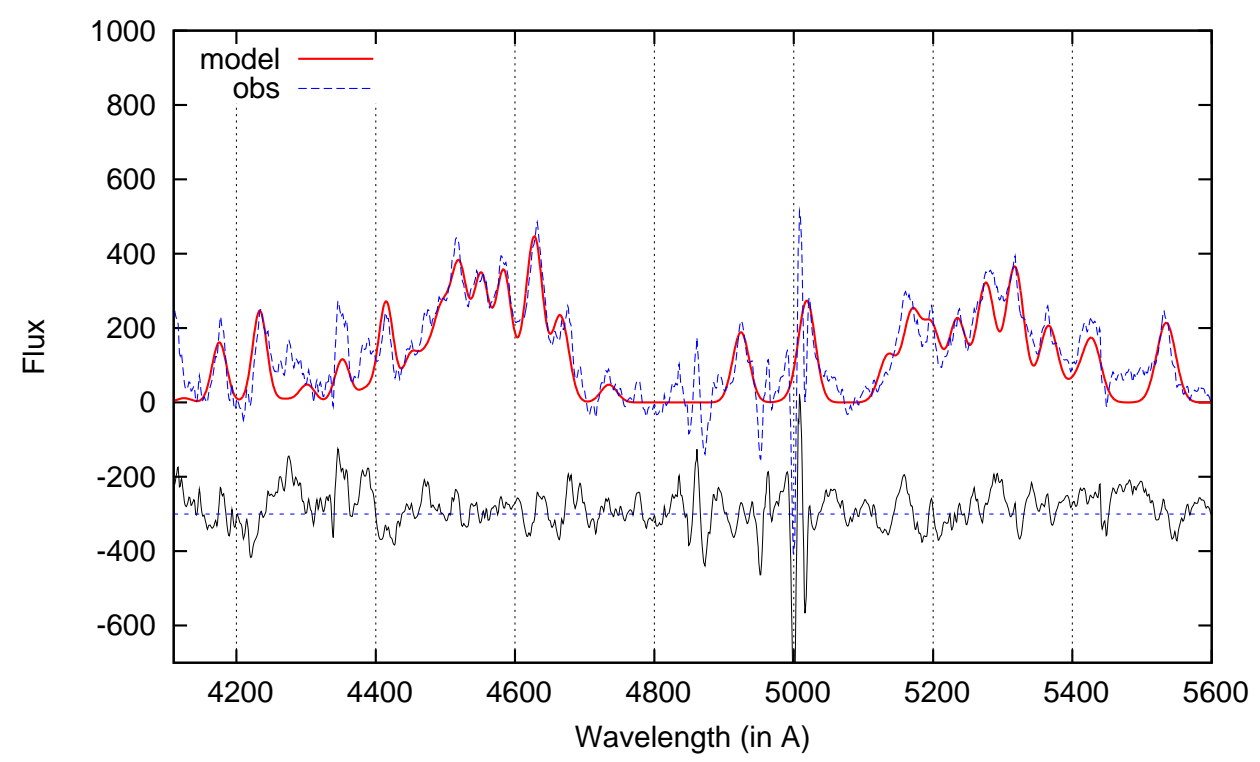

Fig. 15. - An example of the Fe II line emission (date Nov 23, 2001) in $\lambda \lambda 4100-5600 \AA$ cleared from the contamination of other strong lines in the field. The observed (dashed line) and fitted spectra (solid line) are shown. The bottom line represents the residuals. 

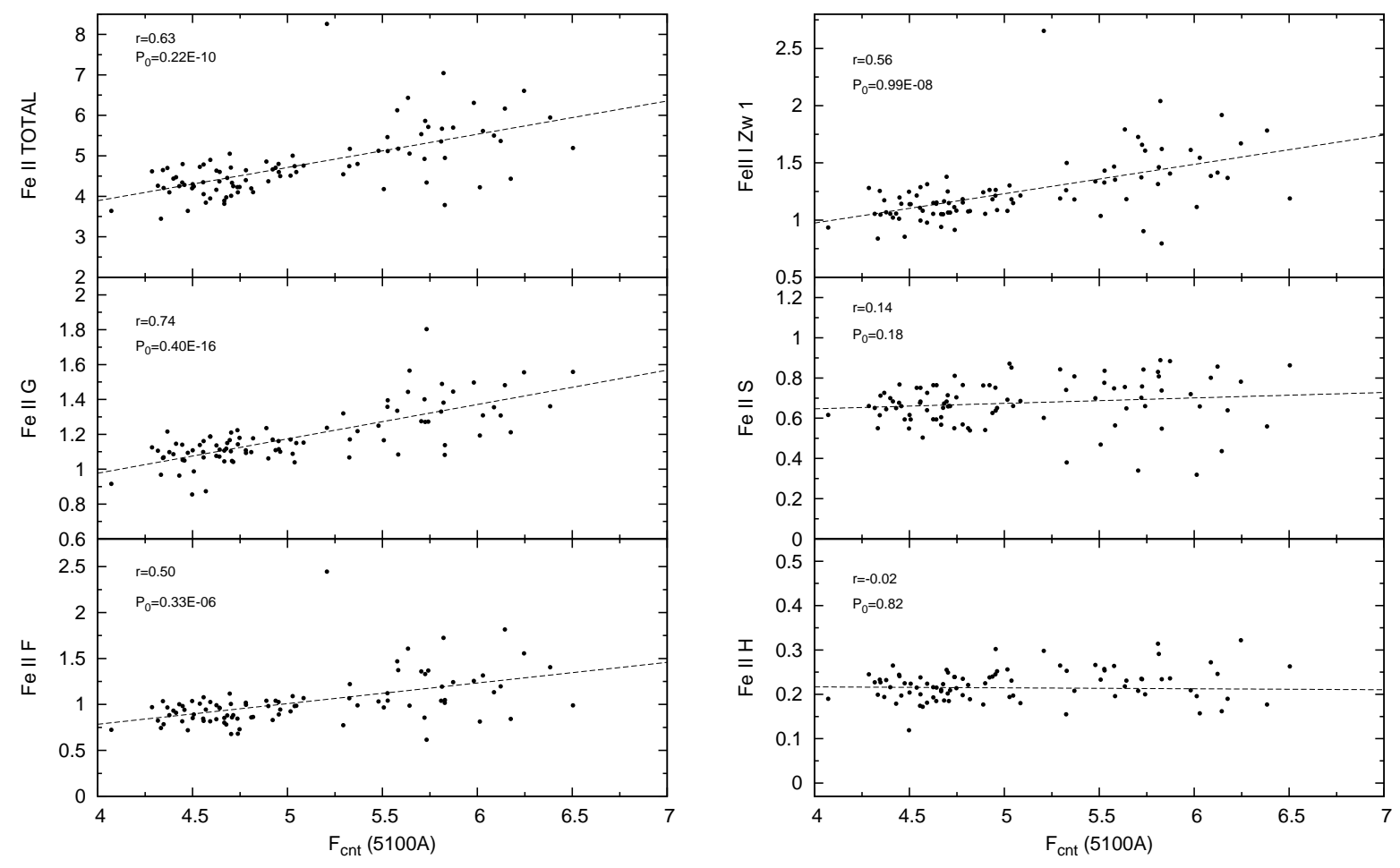

Fig. 16. - Fe II line fluxes (different groups and total fluxes) vs. continuum flux at $5100 \AA$. The correlation coefficient and the corresponding p-value are given in the upper left corner. 


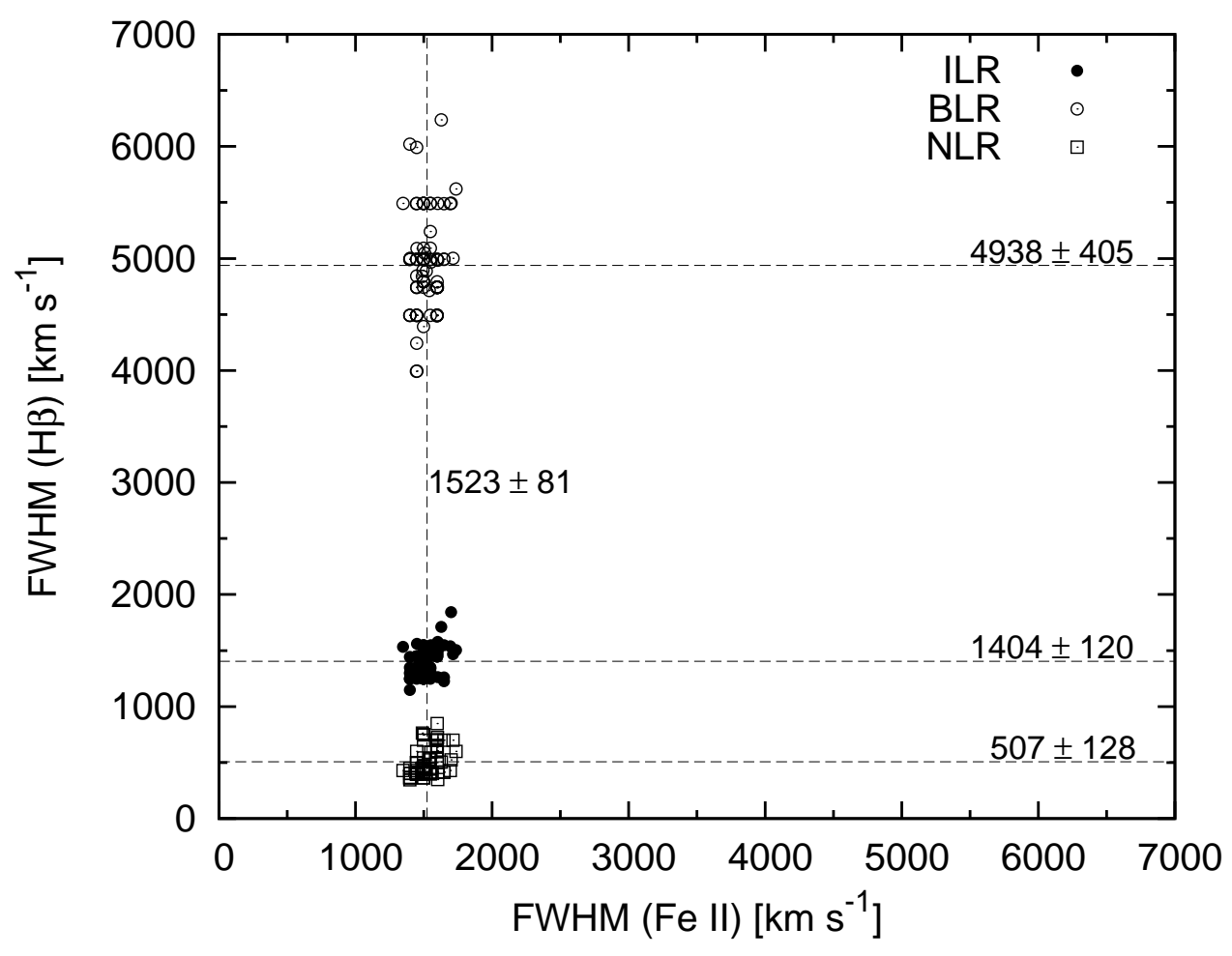

Fig. 17. - Gaussian widths of the Fe II lines compared with the widths of the H $\beta$ ILR component. The vertical line shows the average value of Fe II widths, while the horizontal lines show the average values of the $\mathrm{H} \beta$ BLR, ILR and NLR components. 


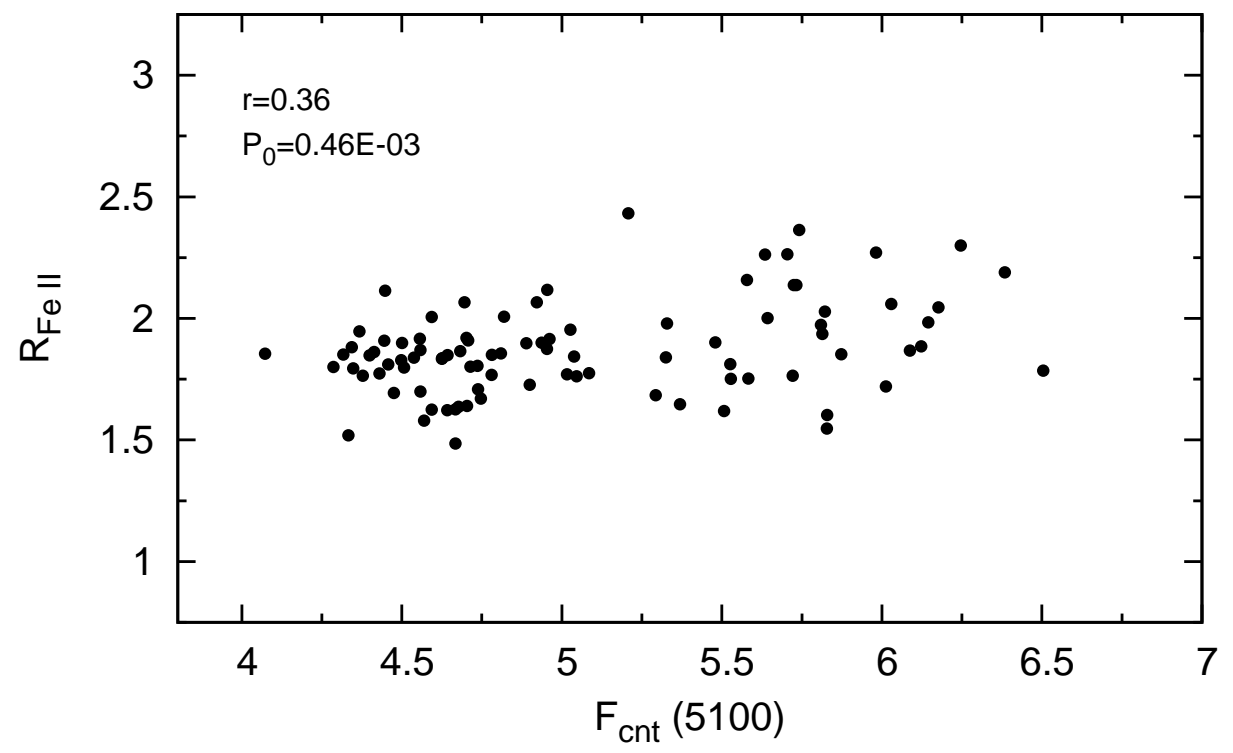

Fig. 18. - $\mathrm{R}_{\mathrm{FeII}}$ plotted against the blue continuum flux (EV1 parameter plane). 
Table 1. Sources of spectroscopic observations.

\begin{tabular}{|c|c|c|c|c|c|c|}
\hline Observatory & Code & Tel.aperture + equipment & Aperture & Focus & No. & Period \\
\hline 1 & 2 & 3 & 4 & 5 & 6 & 7 \\
\hline $\mathrm{SAO}$ (Russia) & $\mathrm{L}(\mathrm{U})$ & $6 \mathrm{~m}+\mathrm{UAGS}$ & $2.0 \times 6.0$ & Prime & 9 & $1999-2001$ \\
\hline $\mathrm{SAO}$ (Russia) & $\mathrm{L}(\mathrm{N})$ & $6 \mathrm{~m}+\mathrm{UAGS}$ & $2.0 \times 6.0$ & Nasmith & 1 & 1999 Oct 09 \\
\hline SAO(Russia) & $\mathrm{Z} 1 \mathrm{~K}$ & $1 \mathrm{~m}+\mathrm{UAGS}+\mathrm{CCD} 1 \mathrm{~K}$ & $4.0 \times 19.8$ & Cassegrain & 19 & $1999-2001$ \\
\hline $\mathrm{SAO}$ (Russia) & $\mathrm{Z} 2 \mathrm{~K}$ & $1 \mathrm{~m}+\mathrm{UAGS}+\mathrm{CCD} 2 \mathrm{~K}$ & $4.0 \times 4.0$ & Cassegrain & 5 & 2006-2009 \\
\hline Gullermo Haro (México) & GHO & $2.1 \mathrm{~m}+\mathrm{B} \& \mathrm{C}$ & $2.5 \times 6.0$ & Cassegrain & 74 & $2000-2007$ \\
\hline SanPedro Martir (México) & SPM & $2.1 \mathrm{~m}+\mathrm{B} \& \mathrm{C}$ & $2.5 \times 6.0$ & Cassegrain & 12 & $2005-2007$ \\
\hline
\end{tabular}

Note. - Col.(1): Observatory. Col.(2): Code assigned to each combination of telescope + equipment used throughout this paper. Col.(3): Telescope aperture and spectrograph. Col.(4): Projected spectrograph entrance apertures (slit width $\times$ slit length in $\operatorname{arcsec})$. Col.(5): Focus of the telescope. Col.(6): Number or spectra obtained. Col.(7): Observation period. 
Table 2. The log of spectroscopic observations.

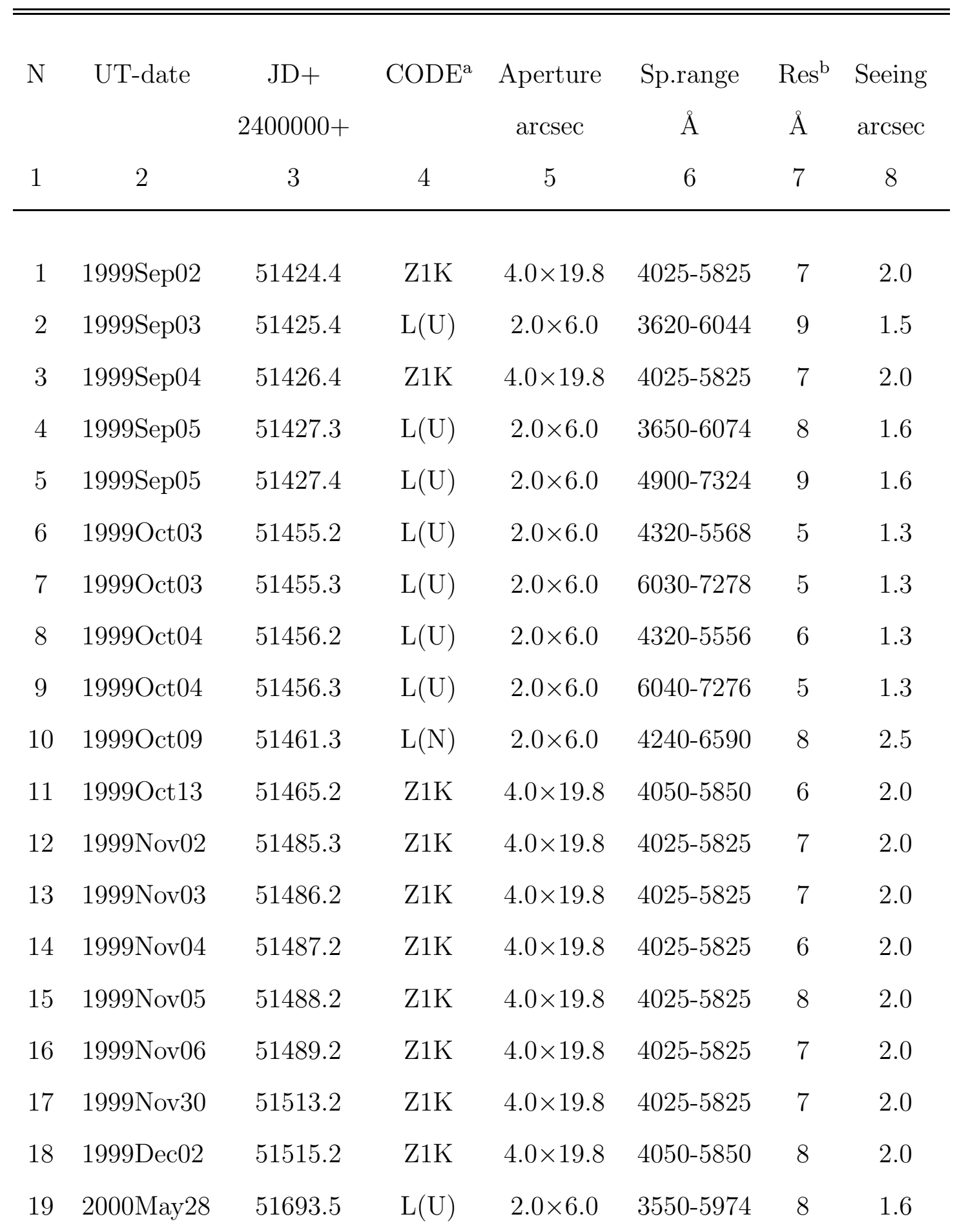


Table 2-Continued

\begin{tabular}{|c|c|c|c|c|c|c|c|}
\hline $\mathrm{N}$ & UT-date & $\mathrm{JD}+$ & $\mathrm{CODE}^{\mathrm{a}}$ & Aperture & Sp.range & $\operatorname{Res}^{\mathrm{b}}$ & Seeing \\
\hline & & $2400000+$ & & $\operatorname{arcsec}$ & $\AA$ & $\AA$ & arcsec \\
\hline 1 & 2 & 3 & 4 & 5 & 6 & 7 & 8 \\
\hline 20 & 2000Jun06 & 51702.4 & Z1K & $4.0 \times 19.8$ & $4020-5820$ & 8 & 3.0 \\
\hline 21 & 2000Jul08 & 51734.4 & Z1K & $4.0 \times 19.8$ & $4050-5850$ & 8 & 3.0 \\
\hline 22 & 2000Jul09 & 51735.4 & $\mathrm{Z} 1 \mathrm{~K}$ & $4.0 \times 19.8$ & $4050-5850$ & 7 & 3.0 \\
\hline 23 & 2000Jul10 & 51736.4 & $\mathrm{Z} 1 \mathrm{~K}$ & $4.0 \times 19.8$ & $4030-5830$ & 7 & 3.0 \\
\hline 24 & $20000 c t 16$ & 51833.7 & $\mathrm{GHO}$ & $2.5 \times 6.0$ & $4000-7300$ & 12 & 2.3 \\
\hline 25 & 2001Aug29 & 52151.5 & $\mathrm{Z} 1 \mathrm{~K}$ & $4.0 \times 19.8$ & $4040-5840$ & 7 & 2.0 \\
\hline 26 & 2001Aug29 & 52151.5 & $\mathrm{Z} 1 \mathrm{~K}$ & $4.0 \times 19.8$ & 5600-7290 & 8 & 2.0 \\
\hline 27 & $20010 \operatorname{ct} 08$ & 52191.2 & $\mathrm{Z} 1 \mathrm{~K}$ & $4.0 \times 19.8$ & $4050-5850$ & 8 & 2.0 \\
\hline 28 & 2001Oct09 & 52192.3 & $\mathrm{Z} 1 \mathrm{~K}$ & $4.0 \times 19.8$ & $4040-5840$ & 7 & 2.0 \\
\hline 29 & $20010 c t 09$ & 52192.4 & $\mathrm{Z} 1 \mathrm{~K}$ & $4.0 \times 19.8$ & 5640-7290 & 11 & 2.0 \\
\hline 30 & 2001Nov23 & 52237.1 & $\mathrm{~L}(\mathrm{U})$ & $2.0 \times 6.0$ & $3600-6024$ & 10 & 3.5 \\
\hline 31 & 2001Nov23 & 52236.6 & $\mathrm{GHO}$ & $2.5 \times 6.0$ & $4200-5960$ & 8 & 2.5 \\
\hline 32 & 2001Nov24 & 52237.6 & $\mathrm{GHO}$ & $2.5 \times 6.0$ & $6000-7360$ & 9 & 1.5 \\
\hline 33 & 2002Aug15 & 52501.8 & $\mathrm{GHO}$ & $2.5 \times 6.0$ & $4270-5840$ & 10 & 2.5 \\
\hline 34 & 2002Aug17 & 52503.9 & $\mathrm{GHO}$ & $2.5 \times 6.0$ & $5700-7460$ & 10 & 2.0 \\
\hline 35 & 2002Nov11 & 52589.7 & $\mathrm{GHO}$ & $2.5 \times 6.0$ & $4300-6060$ & 8 & 4.5 \\
\hline 36 & 2002Nov12 & 52590.7 & $\mathrm{GHO}$ & $2.5 \times 6.0$ & $5700-7460$ & 10 & 2.7 \\
\hline 37 & 2002Nov13 & 52591.7 & $\mathrm{GHO}$ & $2.5 \times 6.0$ & $5700-7460$ & 9 & 2.7 \\
\hline 38 & 2002Nov14 & 52592.7 & $\mathrm{GHO}$ & $2.5 \times 6.0$ & $3800-7100$ & 10 & 2.7 \\
\hline
\end{tabular}


Table 2-Continued

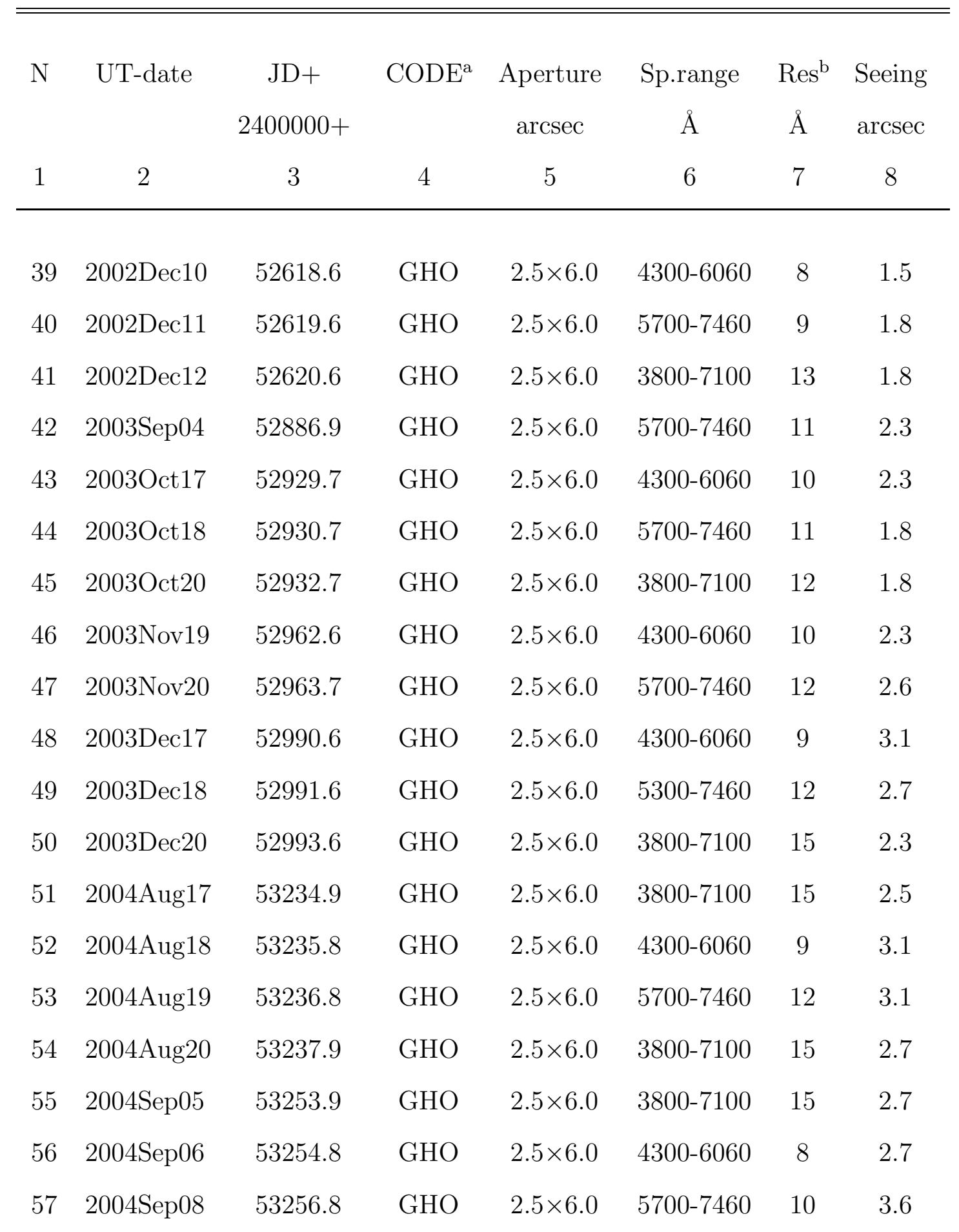


Table 2-Continued

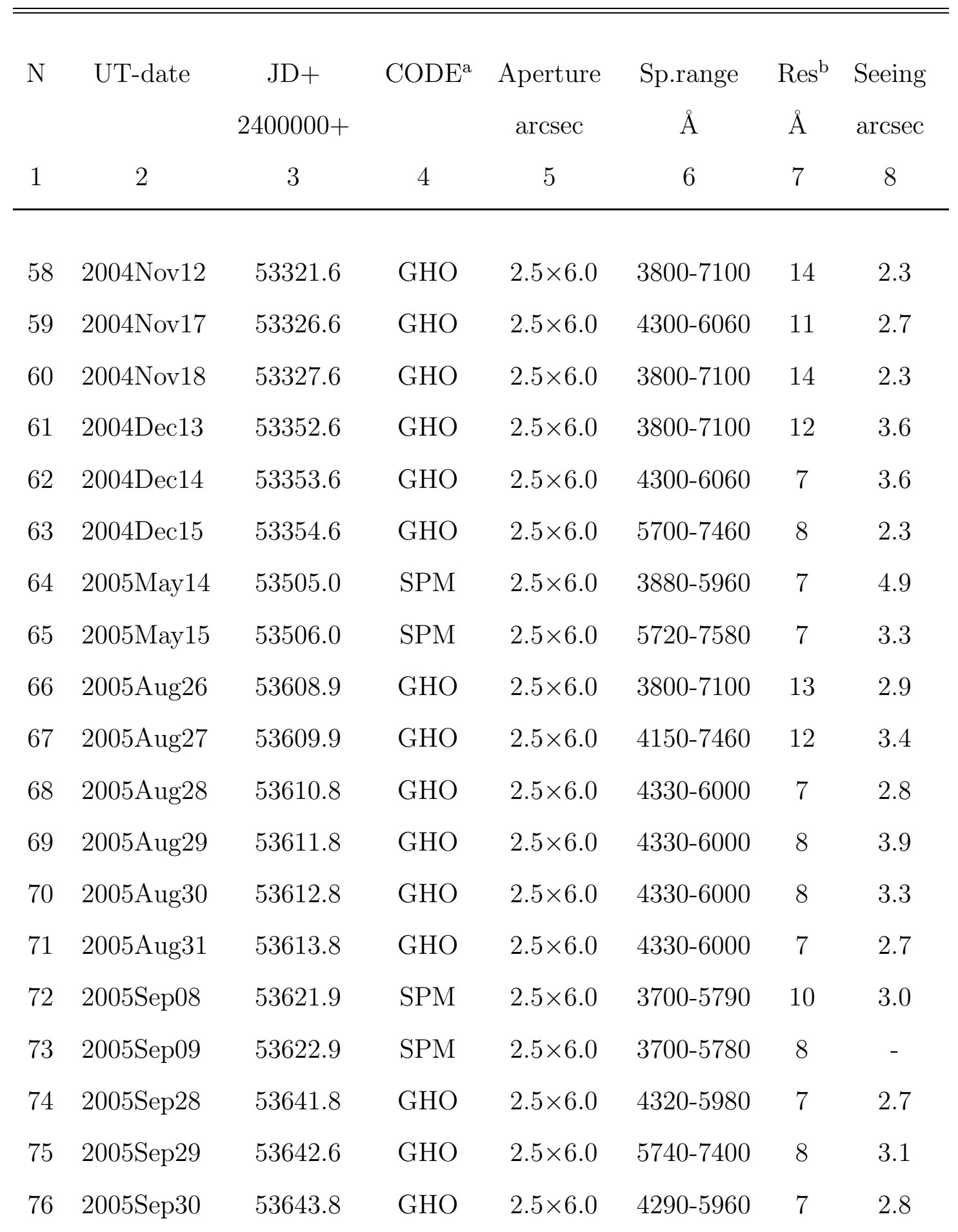


Table 2-Continued

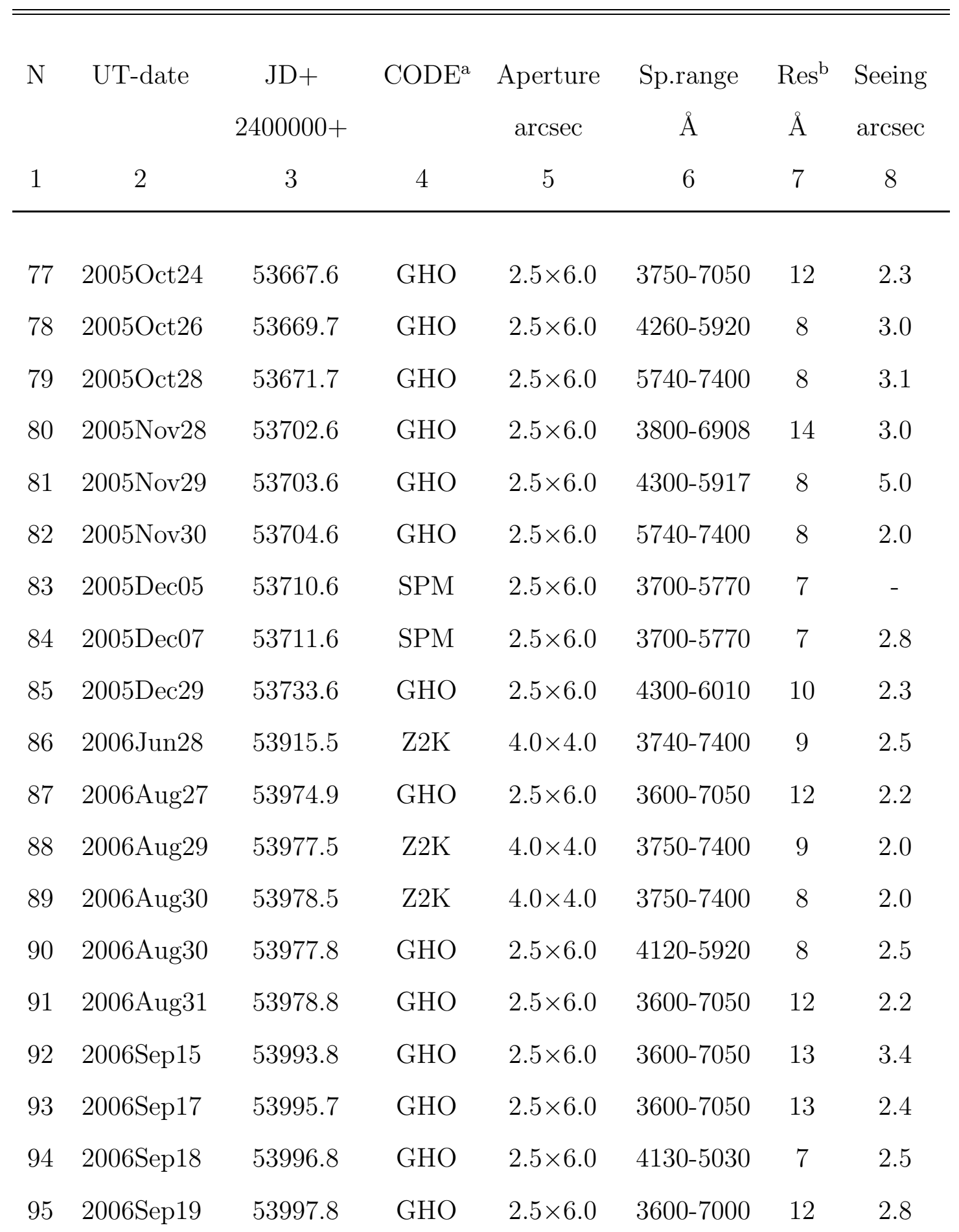


Table 2-Continued

\begin{tabular}{|c|c|c|c|c|c|c|c|}
\hline $\mathrm{N}$ & UT-date & JD+ & $\mathrm{CODE}^{\mathrm{a}}$ & Aperture & Sp.range & $\operatorname{Res}^{b}$ & \\
\hline & & $2400000+$ & & arcsec & $\AA$ & $\AA$ & arcsec \\
\hline 1 & 2 & 3 & 4 & 5 & 6 & 7 & 8 \\
\hline 96 & 2006Sep28 & 54006.7 & SPM & $2.5 \times 6.0$ & $3740-5810$ & 7 & 3.3 \\
\hline 97 & 2006Sep29 & 54007.7 & $\mathrm{SPM}$ & $2.5 \times 6.0$ & $3740-5810$ & 7 & 3.3 \\
\hline 98 & 2006 Oct23 & 54031.7 & $\mathrm{SPM}$ & $2.5 \times 6.0$ & $3700-5900$ & 8 & 2.6 \\
\hline 99 & 2006 Oct 27 & 54035.7 & $\mathrm{GHO}$ & $2.5 \times 6.0$ & $3700-7280$ & 12 & 2.8 \\
\hline 100 & 2006 Oct28 & 54036.7 & $\mathrm{GHO}$ & $2.5 \times 6.0$ & $4230-6040$ & 8 & 2.4 \\
\hline 101 & 2006Oct30 & 54038.7 & $\mathrm{GHO}$ & $2.5 \times 6.0$ & $3700-7270$ & 14 & 2.3 \\
\hline 102 & 2006Oct31 & 54039.7 & $\mathrm{GHO}$ & $2.5 \times 6.0$ & $4160-5960$ & 8 & 2.3 \\
\hline 103 & 2006Nov30 & 54069.6 & $\mathrm{SPM}$ & $2.5 \times 6.0$ & $3680-7560$ & 12 & 4.6 \\
\hline 104 & 2007May22 & 54242.9 & $\mathrm{SPM}$ & $2.5 \times 6.0$ & $3730-5810$ & 8 & 3.0 \\
\hline 105 & 2007May23 & 54244.0 & SPM & $2.5 \times 6.0$ & $3730-5810$ & 8 & 3.2 \\
\hline 106 & 2007Aug10 & 54322.9 & $\mathrm{GHO}$ & $2.5 \times 6.0$ & $3870-7430$ & 11 & 3.0 \\
\hline 107 & 2007Aug11 & 54323.8 & $\mathrm{GHO}$ & $2.5 \times 6.0$ & $4340-6140$ & 7 & 3.2 \\
\hline 108 & 2007Sep03 & 54346.8 & $\mathrm{GHO}$ & $2.5 \times 6.0$ & $4330-6130$ & 7 & 3.6 \\
\hline 109 & 2007Sep04 & 54347.8 & $\mathrm{GHO}$ & $2.5 \times 6.0$ & $4150-5950$ & 7 & 2.6 \\
\hline 110 & 2007Sep07 & 54350.9 & $\mathrm{GHO}$ & $2.5 \times 6.0$ & $3860-7420$ & 12 & 3.0 \\
\hline 111 & 2007 Oct15 & 54388.7 & $\mathrm{GHO}$ & $2.5 \times 6.0$ & $3870-7440$ & 12 & 1.8 \\
\hline 112 & 2007Oct17 & 54390.6 & $\mathrm{GHO}$ & $2.5 \times 6.0$ & $4190-6000$ & 8 & 2.5 \\
\hline 113 & 2007Oct18 & 54391.7 & $\mathrm{GHO}$ & $2.5 \times 6.0$ & $4190-6000$ & 8 & 2.2 \\
\hline 114 & 2007Nov01 & 54405.7 & $\mathrm{GHO}$ & $2.5 \times 6.0$ & $4190-6000$ & 8 & 3.0 \\
\hline
\end{tabular}


Table 2 - Continued

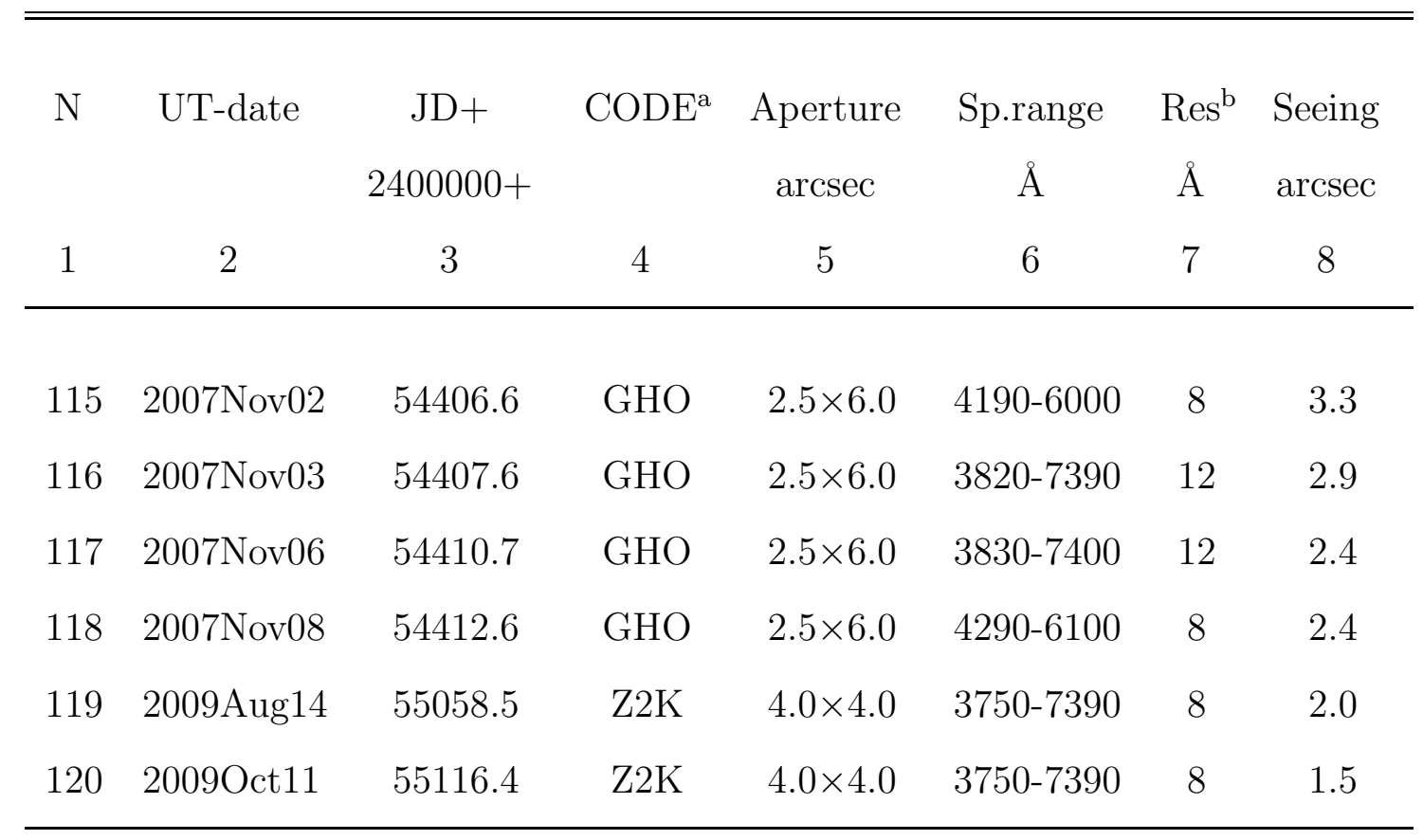

Note. - Col.(1): Number. Col.(2): UT date. Col.(3): Julian date (JD). Col.(4): $\mathrm{CODE}^{\mathrm{a}}$. Col.(5): Projected spectrograph entrance apertures. Col.(6): Wavelength range covered. Col.(7): Spectral resolution ${ }^{\mathrm{b}}$. Col.(8): Mean seeing in arcsec.

${ }^{a}$ Code given according to Table 1,

${ }^{b}$ Resolution determined from [OIII]5007 line, and from [OI]6300 when only red part of the spectrum present. 
Table 3. Flux scale factors for optical spectra.

\begin{tabular}{lcccc}
\hline \hline Sample & Years & Aperture & Scale factor \\
& & $($ arcsec $)$ & $(\varphi)$ & Extended source correction \\
& & & & $\mathrm{G}(\mathrm{g})^{\mathrm{a}}$ \\
\hline L(U,N) & $1999-2010$ & $2.0 \times 6.0$ & 1.089 & -0.130 \\
GHO & $1999-2007$ & $2.5 \times 6.0$ & 1.000 & 0.000 \\
SPM & $2005-2007$ & $2.5 \times 6.0$ & 1.000 & 0.000 \\
Z1K & $1999-2001$ & $4.0 \times 19.8$ & $1.152 \pm 0.013$ & $0.998 \pm 0.368$ \\
Z2K & $2005-2007$ & $4.0 \times 4.0$ & $0.893 \pm 0.052$ & $1.005 \pm 0.548$ \\
GHO* & $1999-2007$ & $2.5 \times 6.0$ & $1.067 \pm 0.048$ & 0.000 \\
\hline
\end{tabular}

*Resolution $15 \AA$

${ }^{\mathrm{a}}$ In units $10^{-15} \operatorname{ergs}^{1} \mathrm{~cm}^{2} \AA^{1}$ 
Table 4. The measured line and continuum fluxes.

\begin{tabular}{|c|c|c|c|c|c|c|}
\hline $\mathrm{N}$ & UT-date & $\begin{array}{c}\mathrm{JD}+ \\
2400000+\end{array}$ & $\begin{array}{c}\mathrm{F}_{\mathrm{cnt}} \pm \sigma \\
10^{-15} \mathrm{erg} \mathrm{cm}^{-2} \mathrm{~s}^{-1} \AA^{-1}\end{array}$ & $\begin{array}{c}\mathrm{F}(\mathrm{H} \alpha) \pm \sigma \\
10^{-13} \mathrm{erg} \mathrm{cm}^{-2} \mathrm{~s}^{-1}\end{array}$ & $\begin{array}{c}\mathrm{F}(\mathrm{H} \beta) \pm \sigma \\
10^{-13} \mathrm{erg} \mathrm{cm}^{-2} \mathrm{~s}^{-1}\end{array}$ & $\begin{array}{l}\text { Fe } \mathrm{II}_{5100-5470} \pm \sigma \\
10^{-13} \mathrm{erg} \mathrm{cm}^{-2} \mathrm{~s}^{-1}\end{array}$ \\
\hline 1 & 2 & 3 & 4 & 5 & 6 & 7 \\
\hline 1 & 1999Sep02 & 51424.4 & $6.145 \pm 0.430$ & - & $2.471 \pm 0.082$ & $2.349 \pm 0.170$ \\
\hline 2 & 1999Sep03 & 51425.4 & $5.479 \pm 0.384$ & - & $2.486 \pm 0.082$ & $2.085 \pm 0.151$ \\
\hline 3 & 1999Sep04 & 51426.4 & $5.507 \pm 0.325$ & - & $2.464 \pm 0.081$ & $2.162 \pm 0.336$ \\
\hline 4 & 1999Sep05 & 51427.3 & $6.246 \pm 0.437$ & $11.963 \pm 0.447$ & $2.589 \pm 0.085$ & $2.696 \pm 0.419$ \\
\hline 5 & 1999Oct03 & 51455.2 & $5.733 \pm 0.214$ & $11.119 \pm 0.416$ & $2.008 \pm 0.221$ & - \\
\hline 6 & 1999Oct04 & 51456.2 & $5.828 \pm 0.218$ & $11.707 \pm 0.438$ & $2.348 \pm 0.258$ & - \\
\hline 7 & 1999Oct09 & 51461.3 & $6.176 \pm 0.231$ & - & $2.459 \pm 0.081$ & $2.111 \pm 0.152$ \\
\hline 8 & 1999Oct13 & 51465.2 & $5.329 \pm 0.199$ & - & $2.478 \pm 0.082$ & $1.948 \pm 0.141$ \\
\hline 9 & 1999Nov02 & 51485.3 & $5.742 \pm 0.215$ & - & $2.233 \pm 0.074$ & $2.496 \pm 0.405$ \\
\hline 10 & 1999Nov03 & 51486.2 & $5.705 \pm 0.213$ & - & $2.253 \pm 0.074$ & $1.798 \pm 0.291$ \\
\hline 11 & 1999Nov04 & 51487.2 & $6.013 \pm 0.225$ & - & $2.348 \pm 0.077$ & $2.170 \pm 0.352$ \\
\hline 12 & 1999Nov05 & 51488.2 & $6.384 \pm 0.239$ & - & $2.478 \pm 0.082$ & - \\
\hline 13 & 1999Nov06 & 51489.2 & $6.030 \pm 0.226$ & - & $2.388 \pm 0.079$ & $2.566 \pm 0.185$ \\
\hline 14 & 1999Nov30 & 51513.2 & $5.635 \pm 0.211$ & - & $2.584 \pm 0.085$ & $2.590 \pm 0.187$ \\
\hline 15 & 1999Dec02 & 51515.2 & $5.583 \pm 0.209$ & - & $2.643 \pm 0.087$ & $1.978 \pm 0.143$ \\
\hline 16 & 2000May28 & 51693.5 & $5.643 \pm 0.211$ & - & $2.447 \pm 0.081$ & $2.794 \pm 0.202$ \\
\hline 17 & 2000Jun06 & 51702.4 & $5.829 \pm 0.218$ & - & $2.822 \pm 0.093$ & $2.016 \pm 0.146$ \\
\hline 18 & 2000Jul08 & 51734.4 & $5.982 \pm 0.431$ & - & $2.578 \pm 0.085$ & $2.493 \pm 0.394$ \\
\hline 19 & 2000Jul09 & 51735.4 & $5.208 \pm 0.375$ & - & $2.745 \pm 0.091$ & $1.950 \pm 0.308$ \\
\hline 20 & 2000Jul10 & 51736.4 & $5.822 \pm 0.419$ & - & $2.836 \pm 0.094$ & $2.669 \pm 0.422$ \\
\hline 21 & 2000 Oct16 & 51833.7 & $5.809 \pm 0.217$ & $11.346 \pm 0.424$ & $2.500 \pm 0.083$ & $2.330 \pm 0.168$ \\
\hline 22 & 2001Nov23 & 52236.6 & $5.722 \pm 0.214$ & - & $2.630 \pm 0.087$ & $2.292 \pm 0.168$ \\
\hline 23 & 2001Nov23 & 52237.1 & $5.725 \pm 0.214$ & - & $2.629 \pm 0.087$ & $2.324 \pm 0.166$ \\
\hline 24 & 2001Nov24 & 52237.6 & - & $10.658 \pm 0.399$ & - & - \\
\hline 25 & 2002Aug15 & 52501.8 & $4.747 \pm 0.178$ & - & $2.472 \pm 0.082$ & $1.836 \pm 0.133$ \\
\hline 26 & 2002Aug17 & 52503.9 & - & $10.094 \pm 0.378$ & - & - \\
\hline 27 & 2002Nov11 & 52589.7 & $5.528 \pm 0.207$ & - & $2.760 \pm 0.091$ & $2.619 \pm 0.189$ \\
\hline 28 & 2002Nov12 & 52590.7 & - & $10.285 \pm 0.385$ & - & - \\
\hline 29 & 2002Nov13 & 52591.7 & - & $10.402 \pm 0.389$ & - & - \\
\hline 30 & 2002Nov14 & 52592.7 & $5.873 \pm 0.220$ & $10.537 \pm 0.394$ & $2.790 \pm 0.092$ & $2.403 \pm 0.173$ \\
\hline
\end{tabular}


Table 4-Continued

\begin{tabular}{|c|c|c|c|c|c|c|}
\hline $\mathrm{N}$ & UT-date & $\begin{array}{c}\mathrm{JD}+ \\
2400000+\end{array}$ & $\begin{array}{c}\mathrm{F}_{\mathrm{cnt}} \pm \sigma \\
10^{-15} \mathrm{erg} \mathrm{cm}^{-2} \mathrm{~s}^{-1} \AA^{-1}\end{array}$ & $\begin{array}{c}\mathrm{F}(\mathrm{H} \alpha) \pm \sigma \\
10^{-13} \mathrm{erg} \mathrm{cm}^{-2} \mathrm{~s}^{-1}\end{array}$ & $\begin{array}{c}\mathrm{F}(\mathrm{H} \beta) \pm \sigma \\
10^{-13} \mathrm{erg} \mathrm{cm}^{-2} \mathrm{~s}^{-1}\end{array}$ & $\begin{array}{l}\text { Fe } \mathrm{II}_{5100-5470} \pm \sigma \\
10^{-13} \mathrm{erg} \mathrm{cm}^{-2} \mathrm{~s}^{-1}\end{array}$ \\
\hline 1 & 2 & 3 & 4 & 5 & 6 & 7 \\
\hline 31 & 2002Dec10 & 52618.6 & $5.294 \pm 0.198$ & - & $2.782 \pm 0.092$ & $2.341 \pm 0.169$ \\
\hline 32 & 2002Dec11 & 52619.6 & - & $9.750 \pm 0.365$ & - & - \\
\hline 33 & 2002Dec12 & 52620.6 & $5.578 \pm 0.209$ & $9.936 \pm 0.372$ & $2.607 \pm 0.086$ & $2.288 \pm 0.165$ \\
\hline 34 & 2003Sep04 & 52886.9 & - & $9.700 \pm 0.363$ & - & - \\
\hline 35 & 2003Oct17 & 52929.7 & $5.016 \pm 0.188$ & - & $2.460 \pm 0.081$ & $1.928 \pm 0.139$ \\
\hline 36 & 2003Oct18 & 52930.7 & - & $8.925 \pm 0.334$ & - & - \\
\hline 37 & 2003Oct20 & 52932.7 & $4.961 \pm 0.186$ & $8.950 \pm 0.335$ & $2.398 \pm 0.079$ & $1.807 \pm 0.130$ \\
\hline 38 & 2003Nov19 & 52962.6 & $4.738 \pm 0.177$ & - & $2.420 \pm 0.080$ & $1.993 \pm 0.144$ \\
\hline 39 & 2003Nov20 & 52963.7 & - & $8.968 \pm 0.335$ & - & - \\
\hline 40 & 2003Dec17 & 52990.6 & $6.504 \pm 0.748$ & - & $3.070 \pm 0.190$ & $2.600 \pm 0.264$ \\
\hline 41 & 2003Dec18 & 52991.6 & - & $11.069 \pm 0.414$ & - & - \\
\hline 42 & 2003Dec20 & 52993.6 & $5.526 \pm 0.636$ & $10.313 \pm 0.386$ & $2.813 \pm 0.174$ & $2.251 \pm 0.229$ \\
\hline 43 & 2004Aug17 & 53234.9 & $5.038 \pm 0.589$ & $9.375 \pm 0.506$ & $2.429 \pm 0.080$ & $1.798 \pm 0.235$ \\
\hline 44 & 2004Aug18 & 53235.8 & $6.123 \pm 0.716$ & - & $2.566 \pm 0.085$ & $2.280 \pm 0.298$ \\
\hline 45 & 2004Aug19 & 53236.8 & - & $8.489 \pm 0.458$ & - & - \\
\hline 46 & 2004Aug20 & 53237.9 & $5.027 \pm 0.588$ & $9.303 \pm 0.502$ & $2.405 \pm 0.079$ & $1.872 \pm 0.245$ \\
\hline 47 & 2004Sep05 & 53253.9 & $5.325 \pm 0.405$ & $10.132 \pm 0.598$ & $2.501 \pm 0.208$ & $1.812 \pm 0.131$ \\
\hline 48 & 2004Sep06 & 53254.8 & $4.781 \pm 0.363$ & - & $2.225 \pm 0.185$ & $1.793 \pm 0.129$ \\
\hline 49 & 2004Sep08 & 53256.8 & - & $9.327 \pm 0.550$ & - & - \\
\hline 50 & 2004Nov12 & 53321.6 & $5.046 \pm 0.189$ & $9.504 \pm 0.355$ & $2.548 \pm 0.084$ & $1.964 \pm 0.142$ \\
\hline 51 & 2004Nov17 & 53326.6 & $5.369 \pm 0.201$ & - & $2.687 \pm 0.089$ & - \\
\hline 52 & 2004Nov18 & 53327.6 & $5.085 \pm 0.190$ & $9.862 \pm 0.369$ & $2.508 \pm 0.083$ & $1.909 \pm 0.138$ \\
\hline 53 & 2004Dec13 & 53352.6 & $6.088 \pm 0.228$ & $11.049 \pm 0.413$ & $2.787 \pm 0.092$ & $2.350 \pm 0.170$ \\
\hline 54 & 2004Dec14 & 53353.6 & $5.814 \pm 0.217$ & - & $2.657 \pm 0.088$ & $2.596 \pm 0.187$ \\
\hline 55 & 2004Dec15 & 53354.6 & - & $10.268 \pm 0.384$ & - & - \\
\hline 56 & 2005May14 & 53505.0 & $4.954 \pm 0.185$ & - & $2.091 \pm 0.069$ & $1.918 \pm 0.138$ \\
\hline 57 & 2005May14 & 53506.0 & - & $10.081 \pm 0.377$ & - & - \\
\hline 58 & 2005Aug26 & 53608.9 & $4.448 \pm 0.166$ & $8.321 \pm 0.691$ & $2.283 \pm 0.075$ & $1.919 \pm 0.139$ \\
\hline 59 & 2005Aug27 & 53609.9 & $4.937 \pm 0.185$ & $9.362 \pm 0.777$ & $2.450 \pm 0.081$ & $1.724 \pm 0.125$ \\
\hline 60 & 2005Aug28 & 53610.8 & $4.559 \pm 0.170$ & - & $2.310 \pm 0.076$ & $1.988 \pm 0.144$ \\
\hline
\end{tabular}


Table 4-Continued

\begin{tabular}{|c|c|c|c|c|c|c|}
\hline $\mathrm{N}$ & UT-date & $\begin{array}{c}\mathrm{JD}+ \\
2400000+\end{array}$ & $\begin{array}{c}\mathrm{F}_{\mathrm{cnt}} \pm \sigma \\
10^{-15} \mathrm{erg} \mathrm{cm}^{-2} \mathrm{~s}^{-1} \AA^{-1}\end{array}$ & $\begin{array}{c}\mathrm{F}(\mathrm{H} \alpha) \pm \sigma \\
10^{-13} \mathrm{erg} \mathrm{cm}^{-2} \mathrm{~s}^{-1}\end{array}$ & $\begin{array}{c}\mathrm{F}(\mathrm{H} \beta) \pm \sigma \\
10^{-13} \mathrm{erg} \mathrm{cm}^{-2} \mathrm{~s}^{-1}\end{array}$ & $\begin{array}{l}\mathrm{Fe} \mathrm{II}_{5100-5470} \pm \sigma \\
10^{-13} \mathrm{erg} \mathrm{cm}^{-2} \mathrm{~s}^{-1}\end{array}$ \\
\hline 1 & 2 & 3 & 4 & 5 & 6 & 7 \\
\hline 61 & 2005Aug29 & 53611.8 & $4.678 \pm 0.175$ & - & $2.360 \pm 0.078$ & $1.880 \pm 0.136$ \\
\hline 62 & 2005Aug30 & 53612.8 & $4.593 \pm 0.172$ & - & $2.268 \pm 0.075$ & $2.035 \pm 0.147$ \\
\hline 63 & 2005Aug31 & 53613.8 & $4.704 \pm 0.176$ & - & $2.289 \pm 0.076$ & $2.160 \pm 0.156$ \\
\hline 64 & 2005Sep08 & 53621.9 & $4.498 \pm 0.168$ & - & $2.118 \pm 0.070$ & $1.543 \pm 0.238$ \\
\hline 65 & 2005Sep09 & 53622.9 & $4.809 \pm 0.180$ & - & $2.128 \pm 0.070$ & $1.921 \pm 0.296$ \\
\hline 66 & 2005Sep28 & 53641.8 & $4.333 \pm 0.162$ & - & $2.270 \pm 0.075$ & $1.708 \pm 0.123$ \\
\hline 67 & 2005Sep29 & 53642.6 & - & $8.359 \pm 0.313$ & - & - \\
\hline 68 & 2005Sep30 & 53643.8 & $4.378 \pm 0.164$ & - & $2.182 \pm 0.072$ & $1.838 \pm 0.133$ \\
\hline 69 & 2005 Oct 24 & 53667.6 & $4.556 \pm 0.170$ & $9.429 \pm 0.353$ & $2.133 \pm 0.070$ & $1.908 \pm 0.138$ \\
\hline 70 & 2005 Oct26 & 53669.7 & $4.457 \pm 0.167$ & - & $2.176 \pm 0.072$ & $1.806 \pm 0.130$ \\
\hline 71 & 2005 Oct 28 & 53671.7 & - & $9.112 \pm 0.341$ & - & - \\
\hline 72 & 2005 Nov 28 & 53702.6 & $4.072 \pm 0.470$ & - & $1.982 \pm 0.239$ & $1.628 \pm 0.321$ \\
\hline 73 & 2005Nov29 & 53703.6 & $4.736 \pm 0.470$ & - & $2.320 \pm 0.239$ & $2.156 \pm 0.425$ \\
\hline 74 & 2005Nov30 & 53704.6 & - & $9.397 \pm 0.351$ & - & - \\
\hline 75 & 2005Dec05 & 53710.6 & $4.714 \pm 0.176$ & - & $2.267 \pm 0.075$ & $1.761 \pm 0.127$ \\
\hline 76 & 2005Dec07 & 53711.6 & $4.507 \pm 0.169$ & - & $2.146 \pm 0.071$ & $1.714 \pm 0.124$ \\
\hline 77 & 2005Dec29 & 53733.6 & $4.475 \pm 0.167$ & - & $2.270 \pm 0.075$ & $1.834 \pm 0.132$ \\
\hline 78 & 2006Aug27 & 53974.9 & $4.538 \pm 0.286$ & $9.399 \pm 0.352$ & $2.384 \pm 0.079$ & $1.942 \pm 0.140$ \\
\hline 79 & 2006Aug29 & 53977.5 & - & $9.254 \pm 0.346$ & - & - \\
\hline 80 & 2006Aug30 & 53977.8 & $4.642 \pm 0.174$ & - & $2.335 \pm 0.077$ & $1.774 \pm 0.128$ \\
\hline 81 & 2006Aug30 & 53978.5 & - & $8.801 \pm 0.329$ & - & - \\
\hline 82 & 2006Aug31 & 53978.8 & $4.558 \pm 0.170$ & $9.337 \pm 0.349$ & $2.273 \pm 0.075$ & $1.877 \pm 0.135$ \\
\hline 83 & 2006Sep15 & 53993.8 & $4.953 \pm 0.185$ & $9.883 \pm 0.370$ & $2.511 \pm 0.083$ & $1.959 \pm 0.141$ \\
\hline 84 & 2006Sep17 & 53995.7 & $4.889 \pm 0.183$ & $9.941 \pm 0.372$ & $2.469 \pm 0.081$ & $2.068 \pm 0.149$ \\
\hline 85 & 2006Sep18 & 53996.8 & $4.899 \pm 0.183$ & - & $2.288 \pm 0.076$ & $1.686 \pm 0.122$ \\
\hline 86 & 2006Sep19 & 53997.8 & $4.626 \pm 0.173$ & $9.352 \pm 0.350$ & $2.352 \pm 0.078$ & $1.916 \pm 0.138$ \\
\hline 87 & 2006Sep28 & 54006.7 & $4.501 \pm 0.168$ & - & $2.156 \pm 0.071$ & $2.011 \pm 0.145$ \\
\hline 88 & 2006Sep29 & 54007.7 & $4.625 \pm 0.173$ & - & $2.200 \pm 0.073$ & $1.951 \pm 0.141$ \\
\hline 89 & 2006 Oct 23 & 54031.7 & $4.819 \pm 0.180$ & - & $1.910 \pm 0.063$ & $2.101 \pm 0.152$ \\
\hline 90 & 2006Oct27 & 54035.7 & $4.643 \pm 0.174$ & $9.319 \pm 0.349$ & $2.305 \pm 0.076$ & $1.916 \pm 0.317$ \\
\hline
\end{tabular}


Table 4-Continued

\begin{tabular}{|c|c|c|c|c|c|c|}
\hline $\mathrm{N}$ & UT-date & $\begin{array}{c}\mathrm{JD}+ \\
2400000+\end{array}$ & $\begin{array}{c}\mathrm{F}_{\mathrm{cnt}} \pm \sigma \\
10^{-15} \mathrm{erg} \mathrm{cm}^{-2} \mathrm{~s}^{-1} \AA^{-1}\end{array}$ & $\begin{array}{c}\mathrm{F}(\mathrm{H} \alpha) \pm \sigma \\
10^{-13} \mathrm{erg} \mathrm{cm}^{-2} \mathrm{~s}^{-1}\end{array}$ & $\begin{array}{c}\mathrm{F}(\mathrm{H} \beta) \pm \sigma \\
10^{-13} \mathrm{erg} \mathrm{cm}^{-2} \mathrm{~s}^{-1}\end{array}$ & $\begin{array}{l}\text { Fe } \mathrm{II}_{5100-5470} \pm \sigma \\
10^{-13} \mathrm{erg} \mathrm{cm}^{-2} \mathrm{~s}^{-1}\end{array}$ \\
\hline 1 & 2 & 3 & 4 & 5 & 6 & 7 \\
\hline 91 & 2006 Oct 28 & 54036.7 & $4.570 \pm 0.171$ & - & $2.280 \pm 0.075$ & $1.515 \pm 0.250$ \\
\hline 92 & 2006 Oct30 & 54038.7 & $4.781 \pm 0.320$ & $9.930 \pm 0.371$ & $2.434 \pm 0.153$ & $1.840 \pm 0.133$ \\
\hline 93 & 2006 Oct31 & 54039.7 & $4.348 \pm 0.291$ & - & $2.225 \pm 0.141$ & $1.810 \pm 0.131$ \\
\hline 94 & 2006Nov30 & 54069.6 & $4.922 \pm 0.184$ & $9.999 \pm 0.374$ & $2.240 \pm 0.074$ & $1.959 \pm 0.141$ \\
\hline 95 & 2007May22 & 54242.9 & $4.702 \pm 0.176$ & - & $2.285 \pm 0.075$ & $1.919 \pm 0.139$ \\
\hline 96 & 2007May23 & 54244.0 & $4.707 \pm 0.176$ & - & $2.209 \pm 0.073$ & $1.839 \pm 0.133$ \\
\hline 97 & 2007Aug10 & 54322.9 & $4.683 \pm 0.175$ & $8.973 \pm 0.336$ & $2.398 \pm 0.079$ & $1.907 \pm 0.138$ \\
\hline 98 & 2007Aug11 & 54323.8 & $4.667 \pm 0.175$ & - & $2.422 \pm 0.080$ & $1.927 \pm 0.139$ \\
\hline 99 & 2007Sep03 & 54346.8 & $4.668 \pm 0.175$ & - & $2.491 \pm 0.082$ & $1.758 \pm 0.177$ \\
\hline 100 & 2007Sep04 & 54347.8 & $4.696 \pm 0.176$ & - & $2.385 \pm 0.079$ & $2.027 \pm 0.204$ \\
\hline 101 & 2007Sep07 & 54350.9 & $4.430 \pm 0.166$ & $9.760 \pm 0.365$ & $2.496 \pm 0.082$ & $1.715 \pm 0.124$ \\
\hline 102 & 2007 Oct15 & 54388.7 & $4.318 \pm 0.161$ & $9.360 \pm 0.350$ & $2.306 \pm 0.076$ & $1.834 \pm 0.132$ \\
\hline 103 & 2007 Oct17 & 54390.6 & $4.446 \pm 0.166$ & - & $2.401 \pm 0.079$ & $1.969 \pm 0.142$ \\
\hline 104 & 2007 Oct 18 & 54391.7 & $4.344 \pm 0.162$ & - & $2.396 \pm 0.079$ & $1.874 \pm 0.135$ \\
\hline 105 & 2007Nov01 & 54405.7 & $4.413 \pm 0.165$ & - & $2.403 \pm 0.079$ & $2.033 \pm 0.147$ \\
\hline 106 & 2007Nov02 & 54406.6 & $4.399 \pm 0.165$ & - & $2.472 \pm 0.082$ & $1.871 \pm 0.135$ \\
\hline 107 & 2007Nov03 & 54407.6 & $4.594 \pm 0.172$ & $9.530 \pm 0.356$ & $2.444 \pm 0.081$ & $1.964 \pm 0.142$ \\
\hline 108 & 2007Oct06 & 54410.7 & $4.367 \pm 0.163$ & $9.973 \pm 0.373$ & $2.414 \pm 0.080$ & $1.870 \pm 0.135$ \\
\hline 109 & 2007Nov08 & 54412.6 & $4.287 \pm 0.160$ & - & $2.332 \pm 0.077$ & $2.002 \pm 0.145$ \\
\hline 110 & 2009Aug14 & 55058.5 & - & $10.712 \pm 0.401$ & - & - \\
\hline 111 & 2009 Oct11 & 55116.4 & - & $12.204 \pm 0.456$ & - & - \\
\hline
\end{tabular}


Table 5. Estimates of the errors for line and line-segment fluxes.

\begin{tabular}{lcccc}
\hline \hline Line & Spectral Region & & & \\
& {$[\AA]($ obs $)$} & {$[\AA]($ rest $)$} & {$[\%]$} & $V_{r}$ region \\
& & & $\mathrm{km} \mathrm{s}^{-1}$ \\
\hline cont 5100 & $5220-5250$ & $5094-5123$ & $3.7 \pm 3.3$ & - \\
cont 6200 & $6320-6370$ & $6168-6216$ & $4.5 \pm 2.2$ & - \\
H $\alpha$ - total & $6640-6810$ & $6480-6646$ & $3.7 \pm 2.2$ & $(-3792 ;+3792)$ \\
H $\beta$ - total & $4936-5030$ & $4817-4909$ & $3.3 \pm 2.5$ & $(-2710 ;+2950)$ \\
Fe II & $5226-5605$ & $5100-5470$ & $7.2 \pm 5.6$ & - \\
H $\alpha$ - blue & $6635-6698$ & $6475-6537$ & $6.8 \pm 6.6$ & $(-4015 ;-1204)$ \\
H $\alpha$ - core & $6698-6752$ & $6537-6589$ & $3.5 \pm 2.1$ & $(-1204 ;+1204)$ \\
H $\alpha$ - red & $6752-6816$ & $6589-6652$ & $5.6 \pm 4.0$ & $(+1204 ;+4015)$ \\
H $\beta$ - blue & $4928-4960$ & $4809-4840$ & $8.0 \pm 4.9$ & $(-3200 ;-1200)$ \\
$\mathrm{H} \beta$ - core & $4960-5001$ & $4840-4880$ & $3.0 \pm 2.9$ & $(-1200 ;+1200)$ \\
$\mathrm{H} \beta$ - red & $5001-5034$ & $4880-4913$ & $8.7 \pm 5.3$ & $(+1200 ;+3200)$ \\
\hline & & & & \\
\hline
\end{tabular}


Table 6. Flares in light curves of the blue continuum, $\mathrm{H} \beta, \mathrm{H} \alpha$, and Fe II emission.

\begin{tabular}{|c|c|c|c|c|c|c|c|c|c|c|}
\hline \multirow[t]{2}{*}{$\mathrm{N}$} & DATE & JD+ & $\mathrm{F}(\mathrm{cnt})^{\mathrm{a}}$ & $\mathrm{dF}(\mathrm{cnt})$ & $\mathrm{F}(\mathrm{H} \beta)^{\mathrm{b}}$ & $\mathrm{dF}(\mathrm{H} \beta)$ & $\mathrm{F}(\mathrm{H} \alpha)^{\mathrm{b}}$ & $\mathrm{dF}(\mathrm{H} \alpha)$ & $\mathrm{F}(\mathrm{Fe} \mathrm{II})^{\mathrm{b}}$ & $\mathrm{dF}(\mathrm{Fe} \mathrm{II})$ \\
\hline & & 2400000 & $(5235) \AA$ & $\%$ & & $\%$ & & $\%$ & & $\%$ \\
\hline \multirow[t]{2}{*}{1} & 2003Dec17 & 52990.6 & 6.5038 & $18 \%$ & 3.070 & $9 \%$ & 11.069 & $7 \%$ & 2.600 & $10 \%$ \\
\hline & 2003Dec20 & 52993.6 & 5.5264 & & 2.813 & & 10.313 & & 2.251 & \\
\hline \multirow[t]{3}{*}{2} & 2004Aug17 & 53234.9 & 5.0378 & $22 \%$ & 2.429 & $6 \%$ & 9.375 & $10 \%$ & 1.798 & $13 \%$ \\
\hline & 2004Aug18 & 53235.8 & 6.1232 & & 2.566 & & 8.489 & & 2.280 & \\
\hline & 2004Aug20 & 53237.9 & 5.0271 & & 2.405 & & 9.303 & & 1.872 & \\
\hline \multirow[t]{2}{*}{3} & 2005 Nov 28 & 53702.6 & 4.0724 & $16 \%$ & 1.982 & $17 \%$ & - & - & 1.628 & $20 \%$ \\
\hline & 2005Nov29 & 53703.6 & 4.7362 & & 2.320 & & - & & 2.156 & \\
\hline \multirow[t]{2}{*}{4} & 2006 Oct 27 & 54035.7 & & & & & & & 1.916 & $17 \%$ \\
\hline & 2006Oct28 & 54036.7 & & & & & & & 1.515 & \\
\hline \multirow[t]{2}{*}{5} & 2006Oct30 & 54038.7 & 4.7813 & $10 \%$ & 2.434 & $9 \%$ & 9.319 & $7 \%$ & 1.840 & $7 \%$ \\
\hline & 2006Oct31 & 54039.7 & 4.3478 & & 2.225 & & 9.930 & & 1.810 & \\
\hline
\end{tabular}

${ }^{\mathrm{a}}$ Continuum flux is in units $10^{-15} \mathrm{erg} \mathrm{cm}^{-2} \mathrm{~s}^{-1} \mathrm{~A}^{-1}$.

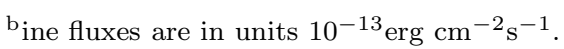


Table 7. Parameters of the continuum and line variabilities.

\begin{tabular}{|c|c|c|c|c|c|c|}
\hline Feature & $\mathrm{N}$ & Region $[\AA]$ & $F(\text { mean })^{\mathrm{a}}$ & $\sigma(F)^{\mathrm{a}}$ & $R(\max / \min )$ & $F(\operatorname{var})$ \\
\hline 1 & 2 & 3 & 4 & 5 & 6 & 7 \\
\hline continuum 5100 & 91 & $5094-5123$ & 5.068 & 0.608 & 1.597 & 0.107 \\
\hline continuum 6300 & 50 & $6168-6216$ & 2.021 & 0.218 & 1.471 & 0.098 \\
\hline $\mathrm{H} \alpha$ - total & 50 & 6480-6646 & 9.856 & 0.878 & 1.467 & 0.079 \\
\hline $\mathrm{H} \beta$ - total & 91 & 4817-4909 & 2.413 & 0.206 & 1.607 & 0.075 \\
\hline Fe II & 87 & $5100-5470$ & 2.029 & 0.280 & 1.844 & 0.096 \\
\hline $\mathrm{H} \alpha$ - blue & 50 & $6475-6537$ & 0.652 & 0.079 & 1.852 & 0.081 \\
\hline $\mathrm{H} \alpha$ - core & 50 & $6537-6589$ & 8.581 & 0.757 & 1.445 & 0.080 \\
\hline $\mathrm{H} \alpha-$ red & 50 & $6589-6652$ & 0.731 & 0.074 & 1.605 & 0.077 \\
\hline $\mathrm{H} \beta$ - blue & 91 & $4809-4840$ & 0.220 & 0.040 & 3.465 & 0.149 \\
\hline $\mathrm{H} \beta$ - core & 91 & $4840-4880$ & 2.008 & 0.152 & 1.566 & 0.064 \\
\hline $\mathrm{H} \beta-$ red & 91 & $4880-4913$ & 0.258 & 0.040 & 2.238 & 0.110 \\
\hline
\end{tabular}

Note. - Col.(1): Analyzed feature of the spectrum. Col.(2): Total number of spectra. Col.(3): Wavelength region (in the rest frame). Col.(4): Mean flux. ${ }^{a}$ Col.(5): Standard deviation ${ }^{\mathrm{a}}$. Col.(6): Ratio of the maximal to minimal flux . Col.(7): Variation amplitude (see text).

${ }^{a}$ Continuum flux is in units $10^{-15} \mathrm{erg} \mathrm{cm}^{-2} \mathrm{~s}^{-1} \mathrm{~A}^{-1}$. and line fluxes and line- 


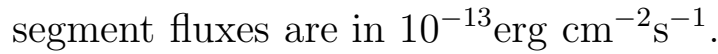


Table 8. Lags and CCF between the continuum and lines.

\begin{tabular}{ccc}
\hline \hline & & \\
LC1-LC2 & lag (days) & CCF \\
\hline & & \\
cnt-H $\beta_{\text {tot }}$ & $3.56_{3.56}^{+27.44}$ & $0.49_{-0.09}^{+0.08}$ \\
cnt-Fe II & $0.02_{2.08}^{+2.02}$ & $0.52_{-0.08}^{+0.08}$ \\
cnt-H $\alpha_{\text {tot }}$ & $4.54_{14.46}^{+5.54}$ & $0.49_{-0.01}^{+0.01}$ \\
\hline
\end{tabular}


Table 9. Line transitions added to the Fe II template given in Tables 1 and 2 of Kovačević et al. (2010). The atomic data are taken from the NIST atomic database.

\begin{tabular}{|c|c|c|}
\hline Fe II multiplet & Transition & Wavelength $[\AA]$ \\
\hline \multirow[t]{15}{*}{ Fe II 27-28 } & $\mathrm{b}^{4} \mathrm{P}_{5 / 2}-\mathrm{z}^{4} \mathrm{~F}_{3 / 2}^{o}$ & 4087.284 \\
\hline & $\mathrm{b}^{4} \mathrm{P}_{5 / 2}-\mathrm{z}^{4} \mathrm{~F}_{5 / 2}^{o}$ & 4122.668 \\
\hline & $\mathrm{b}^{4} \mathrm{P}_{5 / 2}-\mathrm{z}^{4} \mathrm{D}_{3 / 2}^{o}$ & 4128.748 \\
\hline & $\mathrm{b}^{4} \mathrm{P}_{5 / 2}-\mathrm{z}^{4} \mathrm{D}_{5 / 2}^{o}$ & 4173.461 \\
\hline & $\mathrm{b}^{4} \mathrm{P}_{5 / 2}-\mathrm{z}^{4} \mathrm{~F}_{7 / 2}^{o}$ & 4178.862 \\
\hline & $\mathrm{b}^{4} \mathrm{P}_{5 / 2}-\mathrm{z}^{4} \mathrm{D}_{7 / 2}^{o}$ & 4233.172 \\
\hline & $\mathrm{b}^{4} \mathrm{P}_{3 / 2}-\mathrm{z}^{4} \mathrm{~F}_{3 / 2}^{o}$ & 4258.154 \\
\hline & $\mathrm{b}^{4} \mathrm{P}_{3 / 2}-\mathrm{z}^{4} \mathrm{D}_{1 / 2}^{o}$ & 4273.326 \\
\hline & $\mathrm{b}^{4} \mathrm{P}_{3 / 2}-\mathrm{z}^{4} \mathrm{~F}_{5 / 2}^{o}$ & 4296.572 \\
\hline & $\mathrm{b}^{4} \mathrm{P}_{3 / 2}-\mathrm{z}^{4} \mathrm{D}_{3 / 2}^{o}$ & 4303.176 \\
\hline & $\mathrm{b}^{4} \mathrm{P}_{3 / 2}-\mathrm{z}^{4} \mathrm{D}_{5 / 2}^{o}$ & 4351.769 \\
\hline & $\mathrm{b}^{4} \mathrm{P}_{1 / 2}-\mathrm{z}^{4} \mathrm{~F}_{3 / 2}^{o}$ & 4369.411 \\
\hline & $\mathrm{b}^{4} \mathrm{P}_{1 / 2}-\mathrm{z}^{4} \mathrm{D}_{1 / 2}^{o}$ & 4385.387 \\
\hline & $\mathrm{b}^{4} \mathrm{P}_{1 / 2}-\mathrm{z}^{4} \mathrm{D}_{3 / 2}^{o}$ & 4416.830 \\
\hline & $\mathrm{b}^{4} \mathrm{P}_{5 / 2}-\mathrm{z}^{6} \mathrm{~F}_{7 / 2}^{o}$ & 4670.182 \\
\hline \multirow[t]{2}{*}{ Fe II] 55} & $\mathrm{~b}^{2} \mathrm{H}_{9 / 2}-\mathrm{z}^{4} \mathrm{D}_{7 / 2}^{o}$ & 5525.125 \\
\hline & $\mathrm{b}^{2} \mathrm{H}_{11 / 2}-\mathrm{z}^{4} \mathrm{~F}_{9 / 2}^{o}$ & 5534.847 \\
\hline
\end{tabular}


Table 10. The line parameters (w-widths, s-shifts, i-intensity*) from the gaussian best-fitting of $\mathrm{H} \beta$ and Fe II lines for 91 good-resolution spectra.

\begin{tabular}{|c|c|c|c|c|c|c|c|c|c|c|c|c|}
\hline & w NLR & s NLR & i NLR & w ILR & s ILR & i ILR & w BLR & s BLR & i BLR & w Fe II & & i Fe II \\
\hline $2400000+$ & $\mathrm{km} / \mathrm{s}$ & $\mathrm{km} / \mathrm{s}$ & {$[\%]$} & $\mathrm{km} / \mathrm{s}$ & $\mathrm{km} / \mathrm{s}$ & {$[\%]$} & $\mathrm{km} / \mathrm{s}$ & $\mathrm{km} / \mathrm{s}$ & {$[\%]$} & $\mathrm{km} / \mathrm{s}$ & $\mathrm{km} / \mathrm{s}$ & {$[\%]$} \\
\hline 1 & 2 & 3 & 4 & 5 & 6 & 7 & 8 & 9 & 10 & 11 & 12 & 13 \\
\hline 51424.4 & 525 & 36 & 0.21 & 1843 & 0 & 0.35 & 5493 & 0 & 0.44 & 1699 & 77 & 1.98 \\
\hline 51425.4 & 504 & 20 & 0.20 & 1712 & 0 & 0.53 & 6238 & -90 & 0.27 & 1627 & 6 & 1.90 \\
\hline 51426.4 & 429 & 21 & 0.18 & 1534 & 0 & 0.48 & 5491 & 390 & 0.33 & 1347 & 125 & 1.62 \\
\hline 51427.3 & 429 & 21 & 0.15 & 1538 & 0 & 0.48 & 5488 & 150 & 0.37 & 1694 & 19 & 2.30 \\
\hline 51455.2 & 349 & 24 & 0.19 & 1578 & 0 & 0.60 & 5491 & 150 & 0.21 & 1602 & 3 & 2.14 \\
\hline 51456.2 & 346 & 24 & 0.17 & 1443 & 30 & 0.54 & 6021 & 148 & 0.29 & 1398 & 56 & 1.55 \\
\hline 51461.3 & 429 & 21 & 0.21 & 1560 & 0 & 0.57 & 3994 & 240 & 0.22 & 1450 & -24 & 2.04 \\
\hline 51465.2 & 409 & 15 & 0.17 & 1446 & 0 & 0.45 & 4995 & -240 & 0.38 & 1497 & -25 & 1.98 \\
\hline 51485.3 & 409 & 15 & 0.16 & 1498 & 150 & 0.43 & 4992 & 0 & 0.41 & 1498 & 93 & 2.36 \\
\hline 51486.2 & 409 & 15 & 0.15 & 1248 & 30 & 0.42 & 5241 & 90 & 0.43 & 1547 & 0 & 2.26 \\
\hline 51487.2 & 409 & 15 & 0.16 & 1248 & 30 & 0.43 & 4992 & 0 & 0.41 & 1448 & 0 & 1.72 \\
\hline 51488.2 & 409 & 15 & 0.16 & 1447 & 30 & 0.43 & 5491 & 0 & 0.41 & 1448 & 174 & 2.19 \\
\hline 51489.2 & 409 & 15 & 0.15 & 1348 & 30 & 0.45 & 5491 & 0 & 0.40 & 1498 & 0 & 2.06 \\
\hline 51513.2 & 409 & 15 & 0.15 & 1348 & 30 & 0.43 & 5491 & 0 & 0.42 & 1497 & -22 & 2.26 \\
\hline 51515.2 & 409 & 15 & 0.14 & 1348 & 30 & 0.41 & 5990 & -450 & 0.44 & 1448 & 0 & 1.75 \\
\hline 51693.5 & 410 & 101 & 0.16 & 1349 & 30 & 0.46 & 5489 & 300 & 0.39 & 1446 & 79 & 2.00 \\
\hline 51702.4 & 449 & 30 & 0.15 & 1348 & 30 & 0.39 & 4992 & 0 & 0.46 & 1398 & 267 & 1.60 \\
\hline 51734.4 & 450 & 30 & 0.17 & 1349 & 30 & 0.44 & 4998 & -1 & 0.39 & 1499 & 173 & 2.27 \\
\hline 51735.4 & 444 & 30 & 0.14 & 1348 & 30 & 0.36 & 5491 & 0 & 0.51 & 1547 & 0 & 2.43 \\
\hline 51736.4 & 444 & 30 & 0.13 & 1298 & 30 & 0.33 & 5492 & 0 & 0.54 & 1547 & 180 & 2.03 \\
\hline 51833.7 & 593 & 5 & 0.16 & 1548 & -6 & 0.46 & 4968 & 0 & 0.37 & 1552 & -66 & 1.97 \\
\hline
\end{tabular}


Table 10-Continued

\begin{tabular}{|c|c|c|c|c|c|c|c|c|c|c|c|c|}
\hline & w NLR & s NLR & i NLR & w ILR & s ILR & i ILR & w BLR & s BLR & i BLR & w Fe II & s Fe II & i Fe II \\
\hline $2400000+$ & $\mathrm{km} / \mathrm{s}$ & $\mathrm{km} / \mathrm{s}$ & {$[\%]$} & $\mathrm{km} / \mathrm{s}$ & $\mathrm{km} / \mathrm{s}$ & {$[\%]$} & $\mathrm{km} / \mathrm{s}$ & $\mathrm{km} / \mathrm{s}$ & {$[\%]$} & $\mathrm{km} / \mathrm{s}$ & $\mathrm{km} / \mathrm{s}$ & {$[\%]$} \\
\hline 1 & 2 & 3 & 4 & 5 & 6 & 7 & 8 & 9 & 10 & 11 & 12 & 13 \\
\hline 52237.1 & 699 & 0 & 0.20 & 1467 & 60 & 0.45 & 5001 & 0 & 0.35 & 1714 & 21 & 2.14 \\
\hline 52236.6 & 499 & 0 & 0.19 & 1548 & 0 & 0.42 & 4991 & -30 & 0.39 & 1598 & 26 & 1.76 \\
\hline 52501.8 & 540 & 15 & 0.19 & 1548 & 0 & 0.48 & 4992 & -30 & 0.33 & 1497 & -133 & 1.67 \\
\hline 52589.7 & 474 & 0 & 0.19 & 1548 & 0 & 0.42 & 5492 & -30 & 0.39 & 1497 & 17 & 1.75 \\
\hline 52592.7 & 599 & -42 & 0.14 & 1503 & -101 & 0.45 & 5621 & -98 & 0.40 & 1736 & 0 & 1.85 \\
\hline 52618.6 & 444 & 18 & 0.20 & 1529 & -41 & 0.48 & 4893 & -33 & 0.33 & 1494 & 48 & 1.68 \\
\hline 52620.6 & 700 & 1 & 0.17 & 1548 & 0 & 0.38 & 5490 & -30 & 0.45 & 1647 & 1 & 2.16 \\
\hline 52929.7 & 534 & 9 & 0.19 & 1454 & 31 & 0.40 & 4714 & 28 & 0.40 & 1539 & 12 & 1.77 \\
\hline 52932.7 & 703 & 1 & 0.21 & 1549 & 0 & 0.46 & 4492 & 0 & 0.33 & 1597 & 41 & 1.92 \\
\hline 52962.6 & 524 & 0 & 0.20 & 1448 & 30 & 0.41 & 4493 & 30 & 0.39 & 1547 & -59 & 1.71 \\
\hline 52990.6 & 500 & 15 & 0.21 & 1448 & 30 & 0.47 & 3993 & 30 & 0.32 & 1446 & 56 & 1.78 \\
\hline 52993.6 & 849 & 30 & 0.21 & 1547 & 30 & 0.40 & 4993 & 60 & 0.39 & 1597 & 46 & 1.81 \\
\hline 53234.9 & 749 & 27 & 0.21 & 1448 & 0 & 0.41 & 4743 & 0 & 0.37 & 1498 & -49 & 1.84 \\
\hline 53235.8 & 499 & 15 & 0.18 & 1447 & 30 & 0.43 & 4742 & 0 & 0.39 & 1448 & -2 & 1.88 \\
\hline 53237.9 & 749 & 27 & 0.20 & 1447 & 0 & 0.42 & 4795 & 15 & 0.38 & 1497 & -29 & 1.95 \\
\hline 53253.9 & 764 & 27 & 0.21 & 1443 & -6 & 0.41 & 4840 & 20 & 0.38 & 1490 & -34 & 1.84 \\
\hline 53254.8 & 414 & 18 & 0.15 & 1260 & 30 & 0.44 & 4993 & 0 & 0.41 & 1647 & 14 & 1.77 \\
\hline 53321.6 & 749 & 27 & 0.20 & 1448 & 0 & 0.43 & 4791 & 15 & 0.37 & 1498 & -7 & 1.76 \\
\hline 53326.6 & 599 & 15 & 0.20 & 1448 & 30 & 0.42 & 4742 & 0 & 0.38 & 1448 & 135 & 1.65 \\
\hline 53327.6 & 749 & 27 & 0.18 & 1448 & 0 & 0.42 & 4793 & 15 & 0.40 & 1498 & 23 & 1.77 \\
\hline 53352.6 & 649 & 27 & 0.18 & 1548 & -60 & 0.41 & 4793 & 15 & 0.42 & 1597 & -57 & 1.87 \\
\hline 53353.6 & 499 & 15 & 0.20 & 1448 & 0 & 0.42 & 4741 & 0 & 0.38 & 1448 & 62 & 1.94 \\
\hline
\end{tabular}


Table 10-Continued

\begin{tabular}{|c|c|c|c|c|c|c|c|c|c|c|c|c|}
\hline & w NLR & s NLR & i NLR & w ILR & s ILR & i ILR & w BLR & s BLR & i BLR & w Fe II & s Fe II & i Fe II \\
\hline $2400000+$ & $\mathrm{km} / \mathrm{s}$ & $\mathrm{km} / \mathrm{s}$ & {$[\%]$} & $\mathrm{km} / \mathrm{s}$ & $\mathrm{km} / \mathrm{s}$ & {$[\%]$} & $\mathrm{km} / \mathrm{s}$ & $\mathrm{km} / \mathrm{s}$ & {$[\%]$} & $\mathrm{km} / \mathrm{s}$ & $\mathrm{km} / \mathrm{s}$ & {$[\%]$} \\
\hline 1 & 2 & 3 & 4 & 5 & 6 & 7 & 8 & 9 & 10 & 11 & 12 & 13 \\
\hline 53495.0 & 397 & 15 & 0.18 & 1398 & 15 & 0.46 & 4986 & 16 & 0.36 & 1498 & 52 & 2.12 \\
\hline 53608.9 & 699 & 0 & 0.21 & 1497 & 0 & 0.42 & 4742 & 15 & 0.36 & 1597 & -44 & 2.11 \\
\hline 53609.9 & 699 & 0 & 0.21 & 1498 & 0 & 0.42 & 4742 & 15 & 0.37 & 1597 & 3 & 1.90 \\
\hline 53610.8 & 400 & 17 & 0.16 & 1347 & 0 & 0.46 & 4991 & 15 & 0.38 & 1548 & -22 & 1.70 \\
\hline 53611.8 & 404 & 24 & 0.16 & 1353 & -1 & 0.45 & 4886 & 16 & 0.38 & 1518 & -59 & 1.64 \\
\hline 53612.8 & 433 & 19 & 0.17 & 1325 & -6 & 0.43 & 5048 & -34 & 0.40 & 1505 & -92 & 1.62 \\
\hline 53613.8 & 399 & 0 & 0.16 & 1332 & -27 & 0.44 & 5091 & 15 & 0.40 & 1548 & -90 & 1.64 \\
\hline 53620.9 & 465 & -14 & 0.17 & 1337 & -60 & 0.43 & 5091 & 15 & 0.40 & 1497 & -134 & 1.83 \\
\hline 53622.9 & 464 & -15 & 0.18 & 1278 & 0 & 0.42 & 4992 & 15 & 0.40 & 1498 & 2 & 1.86 \\
\hline 53641.8 & 419 & 15 & 0.19 & 1278 & 0 & 0.42 & 4992 & 15 & 0.39 & 1498 & -71 & 1.52 \\
\hline 53643.8 & 414 & 22 & 0.16 & 1225 & 30 & 0.46 & 4992 & 0 & 0.39 & 1648 & -19 & 1.76 \\
\hline 53667.6 & 699 & 0 & 0.21 & 1497 & 0 & 0.42 & 4742 & 15 & 0.36 & 1597 & -87 & 1.92 \\
\hline 53669.7 & 419 & 0 & 0.17 & 1278 & 0 & 0.40 & 4991 & 15 & 0.42 & 1497 & -5 & 1.81 \\
\hline 53702.6 & 699 & 3 & 0.19 & 1298 & 0 & 0.40 & 4991 & 0 & 0.41 & 1498 & -67 & 1.85 \\
\hline 53703.6 & 449 & 12 & 0.19 & 1298 & 0 & 0.44 & 4992 & 0 & 0.37 & 1498 & 61 & 1.81 \\
\hline 53710.6 & 364 & 12 & 0.15 & 1248 & 0 & 0.42 & 4991 & 15 & 0.43 & 1498 & -20 & 1.80 \\
\hline 53711.6 & 364 & 12 & 0.15 & 1248 & 0 & 0.42 & 4992 & 15 & 0.43 & 1497 & 0 & 1.80 \\
\hline 53733.6 & 449 & 12 & 0.16 & 1248 & 0 & 0.47 & 4393 & 60 & 0.37 & 1498 & -119 & 1.69 \\
\hline 53974.9 & 649 & 0 & 0.19 & 1498 & 0 & 0.46 & 4742 & 15 & 0.35 & 1598 & -67 & 1.84 \\
\hline 53977.8 & 399 & 12 & 0.15 & 1248 & 0 & 0.40 & 5491 & -300 & 0.44 & 1498 & -9 & 1.62 \\
\hline 53978.8 & 649 & 0 & 0.19 & 1498 & 0 & 0.46 & 4743 & 15 & 0.35 & 1597 & -37 & 1.87 \\
\hline 53993.8 & 650 & 0 & 0.19 & 1497 & 0 & 0.46 & 4746 & 15 & 0.36 & 1598 & -67 & 1.87 \\
\hline
\end{tabular}


Table 10-Continued

\begin{tabular}{|c|c|c|c|c|c|c|c|c|c|c|c|c|}
\hline & w NLR & s NLR & i NLR & w ILR & s ILR & i ILR & w BLR & s BLR & i BLR & w Fe II & s Fe II & i Fe II \\
\hline $2400000+$ & $\mathrm{km} / \mathrm{s}$ & $\mathrm{km} / \mathrm{s}$ & {$[\%]$} & $\mathrm{km} / \mathrm{s}$ & $\mathrm{km} / \mathrm{s}$ & {$[\%]$} & $\mathrm{km} / \mathrm{s}$ & $\mathrm{km} / \mathrm{s}$ & {$[\%]$} & $\mathrm{km} / \mathrm{s}$ & $\mathrm{km} / \mathrm{s}$ & {$[\%]$} \\
\hline 1 & 2 & 3 & 4 & 5 & 6 & 7 & 8 & 9 & 10 & 11 & 12 & 13 \\
\hline 53995.7 & 650 & 15 & 0.19 & 1497 & 0 & 0.46 & 4741 & 15 & 0.35 & 1598 & -92 & 1.90 \\
\hline 53996.8 & 415 & 18 & 0.16 & 1263 & 30 & 0.43 & 4985 & 0 & 0.41 & 1601 & -7 & 1.73 \\
\hline 53997.8 & 639 & 15 & 0.19 & 1472 & 0 & 0.45 & 4742 & 15 & 0.36 & 1597 & -75 & 1.84 \\
\hline 54006.7 & 364 & 12 & 0.17 & 1248 & 0 & 0.48 & 4492 & 300 & 0.35 & 1397 & 19 & 1.90 \\
\hline 54007.7 & 364 & 12 & 0.18 & 1247 & 0 & 0.48 & 4493 & 300 & 0.34 & 1397 & 6 & 1.83 \\
\hline 54031.7 & 369 & 60 & 0.19 & 1148 & 90 & 0.43 & 4492 & 300 & 0.38 & 1398 & 248 & 2.01 \\
\hline 54035.7 & 639 & 15 & 0.19 & 1473 & 0 & 0.45 & 4742 & 15 & 0.36 & 1597 & -90 & 1.85 \\
\hline 54036.7 & 405 & 20 & 0.18 & 1298 & 0 & 0.42 & 4992 & 0 & 0.40 & 1398 & -8 & 1.58 \\
\hline 54038.7 & 725 & 1 & 0.20 & 1470 & 0 & 0.44 & 4740 & 15 & 0.36 & 1600 & -85 & 1.85 \\
\hline 54039.7 & 401 & 3 & 0.16 & 1296 & 0 & 0.42 & 5001 & -1 & 0.42 & 1399 & -84 & 1.79 \\
\hline 54069.6 & 599 & 0 & 0.19 & 1448 & 30 & 0.45 & 4743 & 15 & 0.37 & 1597 & -57 & 2.07 \\
\hline 54242.9 & 400 & 3 & 0.18 & 1297 & 0 & 0.42 & 5088 & 0 & 0.41 & 1450 & 8 & 1.92 \\
\hline 54244.0 & 399 & 3 & 0.20 & 1298 & 0 & 0.45 & 4842 & 0 & 0.35 & 1447 & -16 & 1.91 \\
\hline 54322.9 & 604 & 0 & 0.20 & 1446 & 30 & 0.46 & 4740 & 15 & 0.35 & 1598 & -26 & 1.86 \\
\hline 54323.8 & 414 & 18 & 0.17 & 1306 & 30 & 0.43 & 4992 & 0 & 0.39 & 1547 & -1 & 1.49 \\
\hline 54346.8 & 399 & 3 & 0.20 & 1298 & 0 & 0.44 & 4493 & 0 & 0.36 & 1448 & -6 & 1.63 \\
\hline 54347.8 & 399 & 3 & 0.19 & 1298 & 0 & 0.41 & 4493 & 0 & 0.40 & 1447 & -2 & 2.07 \\
\hline 54350.9 & 606 & 0 & 0.21 & 1460 & 29 & 0.46 & 4488 & 14 & 0.33 & 1596 & -65 & 1.77 \\
\hline 54388.7 & 599 & 15 & 0.19 & 1448 & 30 & 0.47 & 4493 & 15 & 0.34 & 1598 & -100 & 1.85 \\
\hline 54390.6 & 400 & 3 & 0.20 & 1298 & 0 & 0.43 & 4243 & 150 & 0.37 & 1448 & 15 & 1.91 \\
\hline 54391.7 & 399 & 3 & 0.18 & 1298 & 0 & 0.43 & 4493 & 150 & 0.40 & 1448 & 25 & 1.88 \\
\hline 54405.7 & 399 & 3 & 0.18 & 1298 & 0 & 0.43 & 4493 & 150 & 0.39 & 1447 & -15 & 1.86 \\
\hline
\end{tabular}


Table 10-Continued

\begin{tabular}{|c|c|c|c|c|c|c|c|c|c|c|c|c|}
\hline $\mathrm{JD}+$ & w NLR & s NLR & i NLR & w ILR & s ILR & i ILR & w BLR & s BLR & i BLR & w Fe II & s Fe II & i Fe II \\
\hline $2400000+$ & $\mathrm{km} / \mathrm{s}$ & $\mathrm{km} / \mathrm{s}$ & {$[\%]$} & $\mathrm{km} / \mathrm{s}$ & $\mathrm{km} / \mathrm{s}$ & {$[\%]$} & $\mathrm{km} / \mathrm{s}$ & $\mathrm{km} / \mathrm{s}$ & {$[\%]$} & $\mathrm{km} / \mathrm{s}$ & $\mathrm{km} / \mathrm{s}$ & {$[\%]$} \\
\hline 1 & 2 & 3 & 4 & 5 & 6 & 7 & 8 & 9 & 10 & 11 & 12 & 13 \\
\hline 54406.6 & 399 & 3 & 0.18 & 1298 & 0 & 0.43 & 4493 & 150 & 0.39 & 1448 & -22 & 1.85 \\
\hline 54407.6 & 599 & 15 & 0.21 & 1448 & 30 & 0.45 & 4493 & 15 & 0.34 & 1597 & -49 & 2.01 \\
\hline 54410.7 & 607 & 14 & 0.20 & 1451 & 30 & 0.45 & 4495 & 16 & 0.35 & 1595 & -71 & 1.95 \\
\hline 54412.6 & 399 & 3 & 0.17 & 1297 & 0 & 0.40 & 4993 & 0 & 0.42 & 1448 & 3 & 1.80 \\
\hline mean & $507 \pm 128$ & $13 \pm 16$ & $0.18 \pm 0.02$ & $1404 \pm 120$ & $9 \pm 28$ & $0.44 \pm 0.04$ & $4938 \pm 405$ & $25 \pm 109$ & $0.38 \pm 0.05$ & $1523 \pm 81$ & $-2 \pm 74$ & $1.87 \pm 0.19$ \\
\hline
\end{tabular}

${ }^{*}$ The intensity is given as a ratio to the total $\mathrm{H} \beta$ [in \%], i.e. for Fe II it is the parameter $\mathrm{R}_{\mathrm{Fe}}$. 